Universidade de São Paulo

Instituto de Física

\title{
Transições de fase em modelos populacionais com desordem espacial e temporal
}

\author{
Alexander Hideki Oniwa Wada
}

Orientador: Mário José de Oliveira

Tese de doutorado apresentada ao Instituto de Física como requisito parcial para a obtenção do título de Doutor em Ciências.

Banca Examinadora:

Prof. Dr. Mário José de Oliveira (IFUSP)

Prof. Dr. André de Pinho Vieira (IFUSP)

Prof. Dr. José Abel Hoyos Neto (IFSC)

Prof. Dr. Jürgen Fritz Stilck (UFF)

Prof. Dr. Silvio da Costa Ferreira Junior (UFV)

\section{São Paulo}

2019 
FICHA CATALOGRÁFICA

Preparada pelo Serviço de Biblioteca e Informação do Instituto de Física da Universidade de São Paulo

Wada, Alexander Hideki Oniwa.

Transições de fase em modelos populacionais com desordem espacial e temporal. São Paulo, 2019.

Tese (Doutorado) - Universidade de São Paulo. Instituto de Física. Depto. de Física Geral.

Orientador: Prof. Dr. Mário José de Oliveira

Área de Concentração: Física Estatística

Unitermos: 1. Mudança de fase; 2. Propriedade dos sólidos; 3. Mecânica estatística clássica.

USP/IF/SBI-027/2019 


\title{
University of São Paulo
}

Physics Institute

\section{Phase transitions in biological population models with spatial and temporal disorder}

\author{
Alexander Hideki Oniwa Wada
}

Supervisor: Mário José de Oliveira

Thesis submitted to the Physics Institute of the University of São Paulo in partial fulfillment of the requirements for the degree of Doctor of Science.

Examining Committee:

Prof. Dr. Mário José de Oliveira (IFUSP)

Prof. Dr. André de Pinho Vieira (IFUSP)

Prof. Dr. José Abel Hoyos Neto (IFSC)

Prof. Dr. Jürgen Fritz Stilck (UFF)

Prof. Dr. Silvio da Costa Ferreira Junior (UFV) 


\section{Agradecimentos}

Agradeço à Fapesp (processos 2015/02100-8 e 2017/08631-0) e ao CNPq pelo apoio financeiro.

Agradeço ao meu orientador Dr. Mário José de Oliveira, pois sem sua orientação nada do que segue seria possível. Sob sua orientação não só aprendi muita Física, mas também pude melhorar muitas outras habilidades necessárias para um pesquisador.

Agradeço também ao Dr. Thomas Vojta por me receber em seu grupo de pesquisa e me orientar durante o estágio no exterior. A supervisão do Dr. Vojta também foi essencial para este trabalho e a experiência de trabalhar no seu grupo de pesquisa contribuiu muito para a minha formação.

Por fim agradeço a minha família e meus amigos pelo apoio durante esta jornada. Apesar de todos serem de grande importância, gostaria de agradecer especialmente a minha amiga Carolina Feher da Silva pela ajuda com linguagens de programação. 
Resumo

Nesta tese estudamos os efeitos da desordem espacial e temporal na transição de fase entre a sobrevivência e extinção de populações biológicas. Na primeira parte estudamos um modelo epidemiológico com quatro estados. Apesar deste modelo não conter desordem, concluímos que seu comportamento crítico é o mesmo do processo de contato com desordem (espacial) quenched. Na segunda parte estudamos o movimento Browniano fracionário refletido, onde vimos que a combinação dos efeitos do ruído com correlações de longo alcance e a parede refletora cria uma singularidade em lei de potência na densidade de probabilidade da posição do caminhante. Por fim, estudamos a equação logística com desordem temporal através do mapeamento no movimento Browniano fracionário refletido. Neste último estudo vimos como as correlações de longo alcance mudam o comportamento crítico deste sistema.

Palavras-chave: Modelos para populações biológicas, desordem espacial, desordem temporal, fase de Griffiths, percolação direcionada, percolação dinâmica 


\section{Abstract}

We have studied the efects of spatial and temporal disorder at the phase transition between survival and extinction of biological populations. In the first part we studied a four states biological population model. Despite having no disorder, we have seen that its critical behavior is the same of the contact process with (spatial) quenched disorder. In the second part, we studied the reflected fractional Brownian motion, where the interplay between the correlated noise and the reflecting wall results in a power-law singularity in the probability density of the position of the walker. Finally, we deduced the critical properties of the logistic equation with temporal disorder by mapping it onto the reflected fractional Brownian motion. This mapping allow us to understand how long-range correlations change the critical behavior of this system.

Keywords: Biological population model, spatial disorder, temporal disorder, Griffiths phase, directed percolation, dynamical percolation 


\section{Lista de Figuras}

2.1 Exemplo da dinâmica DP num modelo onde o fluído está sob ação da gravidade e apenas pode cair. Os sítios azuis estão ocupados (contém líquido) e os brancos estão vazios. As linhas contínuas e tracejadas são as ligações abertas e fechadas, por onde o líquido pode ou não percolar. 8

2.2 Transições DP em $1+1$ dimensões . . . . . . . . . . . . . . . 8

2.3 Esboço do diagrama de fase para um modelo semelhante ao SIR com reinfecção. O círculo vermelho corresponde a quando as taxas de infecção e reinfecção são idênticas. . . . . . . . . . . . . . . . . . . 9

2.4 Exemplo da divisão em blocos de Kadanoff. . . . . . . . . . . . . . . 10

3.1 Transições possíveis do modelo SEI e SIR. . . . . . . . . . . . . 22

3.2 Relação entre o estado estacionário do modelo SEI e a percolação. . . . 24

4.1 Reações do modelo EQE. Os estados são suscetível $S$, imune $U$, exposto

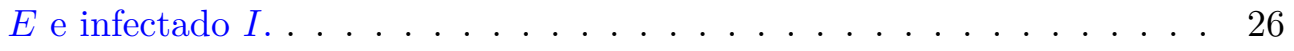

4.2 Dinâmicas separadas do modelo de quatro estados. A primeira parte ocorre com probabilidade $e$, e a segunda com $1-e . \ldots . \ldots 30$

4.3 Simulações iniciadas a partir de uma única semente para $L=8192$, $p=0.8$ e $e=1$. As curvas são médias sobre um número de configurações de desordem que varia de $2 \times 10^{5}$ até $5 \times 10^{6}$. As incertezas são menores

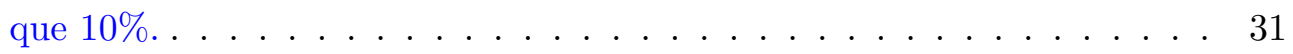

4.4 Gráfico de $P$ por $N_{I}$ para $L=8192 \ldots \ldots \ldots \ldots \ldots \ldots$. . . . . . . 32

4.5 Gráfico de $\xi^{2}$ por $N_{I}$ para $L=8192 \ldots \ldots \ldots \ldots$. . . . . . . 33

4.6 Gráfico de $\xi 2$ por $P$ para $L=8192 \ldots \ldots \ldots \ldots \ldots$

4.7 Ajuste de $t^{b}$ para $p=0.70$ e $p=0.98$. A linha rosa mostra o ajuste. . . 36 
4.8 Diagrama de fase do DCP. Os pontos vermelhos indicam os valores de $\lambda_{c}(p)$, o degradê preto simulação estacionárias com $L=128$ e transiente de $10^{6}$, a linha vertical azul o ponto crítico da percolação $(0.59274 \ldots)$ e a linha rosa a aproximação de pares. . . . . . . . . . . . . . . . .

4.9 Simulações no estado estacionário de $\rho$ e $\xi$ vs. $t$, com tamanho do sistema $L=2048$. Cada curva é uma média de 20 até 40 configurações de desordem. A linha rosa no gráfico de $\rho$ mostra o valor adotado para as médias. . . . . . . . . . . . . . . . . . 38

4.10 Ajuste de $\beta$ obtendo $\beta=1.11(6) \ldots \ldots \ldots \ldots$

4.11 Simulações no ponto multi-crítico $\lambda_{c}\left(p_{c}\right)=3.10(1) \operatorname{com} L=2048$, tempos da ordem de $10^{8}$, cerca de 100 configurações de desordem e $\rho_{0}$ a densidade de sítios iniciais infectados. A linha azul indica o fit da função (4.39)

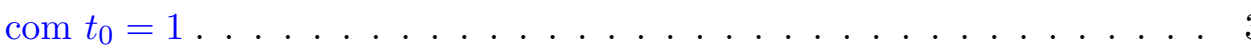

4.12 Gráfico do número de sítios ativos $N_{I}$, probabilidade de sobrevivência $P$, raio médio $R$, e do número de sítios expostos $N_{E}$. O tamanho do sistema é $L=2048$ e cada curve é uma média sobre $1.8 \times 10^{7}$ realizações. As incertezas são menores que o tamanho dos símbolos. . . . . . . . . . . . 41

4.13 Expoentes críticos em função do inverso do tamanho do sistema $L^{-1}$. As linhas vermelhas são extrapolações lineares para $L \rightarrow \infty$. . . . . . . . . 42

5.1 Média e raiz quadra do segundo momento da posição $x$ do movimento Browniano fracionário refletido na transição de regimes $(v=0)$ para diversos valores do expoente de difusão anômala $\alpha$. As linhas tracejadas e sólidas indicam ajustes em lei de potência. A linha sólida amarela indica o comportamento de difusão normal $\langle x\rangle \sim t$ com prefator arbitrário. A

5.2 Densidade de probabilidade $P(x, t)$ da posição do caminhante $x$ no tempo $t$ em escala log-linear para o expoente de difusão anômala $\alpha=1.8$ (que corresponde a superdifusão ou correlações positivas). Inset: comparação entre as densidades de probabilidade do movimento Browniano fracionário não confinado e refletido no tempo $t=16384$. A incerteza é menor que o tamanho dos pontos. . . . . . . . . . . . . . . . . . 49 
5.3 Gráfico de $\left\langle x^{2}\right\rangle^{1 / 2} P$ vs. $x /\left\langle x^{2}\right\rangle^{1 / 2}$ em escala log-log em diversos instantes de tempo. Os painéis (a) e (b) mostram simulações para $\alpha=0.8$ (subdifusão) e 1.8 (superdifusão), respectivamente. Inset: $\left\langle x^{2}\right\rangle^{1 / 2} P$ vs. $x^{2} /\left\langle x^{2}\right\rangle$ em escala log-linear de forma que o comportamento de uma gaussiana se assemelhe a uma linha reta. As incertezas são da ordem do tamanho dos símbolos. . . . . . . . . . . . . . . . . . . . . . . 50

5.4 Densidade de probabilidade $P$ vs. $x$ em escala log-log para diversos valores do expoente de difusão anômala $\alpha$. As incertezas são menores que o tamanho do ponto. . . . . . . . . . . . . . . . . . 51

5.5 Expoente da singularidade (5.16) $\kappa$ em função do expoente de difusão anômala $\alpha$. Os quadrados representam os valores do expoente $\kappa$ e a linha sólida a função $2 / \alpha-2$. Os valores do expoente $\kappa$ para $\alpha=0.4,0.5,0.6$ e 0.7 apresentam grande variação com o tempo e foram extrapolados em função de $(\ln t)^{-2}$ conforme o inset. Os círculos verdes para estes mesmos valores de $\alpha$ representam o valor de $\kappa$ no maior tempo de simulação (sem extrapolação)

5.6 Trajetórias do movimento Browniano refletido nos casos de subdifusão ( $\alpha=0.5)$, difusão normal $(\alpha=1.0)$ e superdifusão $(\alpha=1.5)$. . . . . .

5.7 (a, b, c) Ruído fracionário gaussiano $\xi_{n}$ nos casos de superdifusão $(\alpha>1)$, difusão normal $(\alpha=1)$ e subdifusão $(\alpha<1)$. (d, e, f) Sinal do ruído fracionário gaussiano. . . . . . . . . . . . . . .

5.8 Densidade de probabilidade no regime localizado para $\alpha=1$ (ruído descorrelacionado) e diferentes implementações da parede refletora. . 57

5.9 (a) Posição média $\langle x\rangle$ em função do tempo no regime balístico $(v>0)$, na transição de regime $(v=0)$ e no regime localizado $(v<0)$. No regime balístico $\langle x\rangle$ tende assintoticamente ao movimento balístico $v t$, representado pelas linhas tracejadas. Na transição de regime observamos o comportamento de difusão anômala $\langle x\rangle \sim t^{\alpha / 2}$. No regime localizado $\langle x\rangle$ atinge um valor finito. (b) Simulações mais detalhadas no regime localizado. As incertezas são menores que o tamanho dos símbolos. . . . 58

5.10 (a) Posição estacionária $x_{\text {st }}$ em função da distância ao ponto crítico $|v|$. (b) Tempo de correlação $t_{x}$ vs. $|v|$. (c) Expoentes extraídos dos dados dos painéis (a) e (b). As linhas sólidas indicam os expoentes preditos $\nu_{\|}=2 /(2-\alpha)$ e $\beta_{x}=\alpha /(2-\alpha)$ de acordo com as equações (5.18) e (5.20). As incertezas são da ordem do tamanho dos pontos. . . . . . . . 58 
5.11 (a,b) Densidade de probabilidade em função do tempo no regime localizado, para os regimes super e sub difusivos e $v$ fixo. Em ambos os casos, as densidades de probabilidade para tempos superiores a 524288 concordam dentro das incertezas, portanto podem ser consideradas estacionárias. (c, d) Densidade de probabilidade estacionária $P_{\text {st }}$ para diversos valores de $v$. As incertezas são da ordem do tamanho dos símbolos. . . . . . . . 59

$5.12(\mathrm{a}, \mathrm{b})$ Densidade de probabilidade em função do tempo no regime localizado, para os regimes super e sub difusivos e $v$ fixo. Em ambos os casos as densidades de probabilidade para tempos superiores a 524288 concordam dentro das incertezas, portanto podem ser consideradas estacionárias. $(\mathrm{c}, \mathrm{d})$ Densidade de probabilidade estacionária $P_{\text {st }}$ para diversos valores de $v$. As incertezas são da ordem do tamanho dos símbolos. . . . . . . . .

5.13 Densidade de probabilidade estacionária $P_{\text {st }}$ vs. $x^{2-\alpha}$ para diversos valores de $|v|$ e $\alpha=1.2$ e 0.8 . As linhas sólidas são ajustes de exponenciais $e^{-b x^{2-\alpha}}$. As incertezas, se não mostradas, são menores que $10 \% . \quad$. . . .

5.14 (a) Expoente $b$ do ajuste $P_{\text {st }} \sim e^{-b x^{2-\alpha}}$ vs. $|v|$. As linhas sólidas são ajustes de leis de potência. (b) Expoentes extraídos a partir de ajustes de leis de potência dos dados do painel (a) em função de $|v|$. Os expoentes concordam com a predição $b \sim|v|^{\alpha}$. As incertezas são da ordem do tamanho dos símbolos. . . . . . . . . . . . . . . . .

5.15 (a) Expoente $b$ do ajuste $P_{\mathrm{st}} \sim e^{-b x^{2-\alpha}}$ vs. $|v|$. As linhas sólidas são ajustes de leis de potência. (b) Expoentes extraídos a partir dos ajustes de leis de potência nos dados do painel (a) em função de $|v|$. Os expoentes concordam com a predição $b \sim|v|^{\alpha}$. As incertezas são da ordem do tamanho dos símbolos. . . . . . . . . . . . . . . . .

5.16 (a, b c c) Densidade de probabilidade em diversos instantes de tempo para $v=0.5$. As linhas sólidas representam a densidade de probilidade (5.11) para o caso não confinado. (d, e) Curtose e assimetria da densidade de probabilidade no caso refletido. . . . . . . . . . . . . . .

6.1 Esquerda: evolução logística da densidade de indivíduos $\rho$ dada pela equação (6.2). Se $\lambda>\mu$ a população sobrevive atingindo um valor estacionário de $\rho$. Se $\mu>\lambda$ a população decai exponencialmente. Entre a extinção e sobrevivência da população, em $\mu=\lambda$, temos a transição de fase onde $\rho \sim t^{-1}$. Direita: exemplo da desordem temporal utilizada. O eixo $y$ representa as taxas $\mu_{n}$ ou $\lambda_{n}$, e o eixo $x$ o tempo. . . . . . 66 
6.2 Comportamento qualitativo da densidade média $\langle\rho\rangle$ perto to ponto crítico $\mu_{c}=\lambda=128$ para $\gamma=1.5$. As incertezas são da ordem do tamanho dos

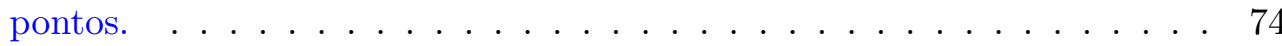

6.3 Densidade de probabilidade $P$ em função da posição $x=-\ln \rho$. Os painéis $(\mathrm{a}, \mathrm{b}),(\mathrm{c}, \mathrm{d})$ e $(\mathrm{e}, \mathrm{f})$ mostram os casos de correlações positivas $(\gamma<1)$, descorrelacionado $(\gamma=1)$ e negativas $(\gamma>1)$. As incertezas são da ordem do tamanho dos pontos. . . . . . . . . . . . . . 76

6.4 (a) Logaritmo da densidade típica $\langle x\rangle=-\ln \rho_{\text {typ }}$ e (b) densidade média $\langle\rho\rangle$ vs. tempo $t$ no ponto crítico $\mu=\mu_{c}=\lambda=128$ para diversos valores de $\gamma$. As linhas sólidas são ajustes das leis de potência $\langle x\rangle \sim t^{\phi}(6.26) \mathrm{e}$ $\langle\rho\rangle \sim t^{-\delta}$ (6.25). As incertezas são menores que o tamanho dos pontos.

6.5 Expoentes críticos $\phi$ e $\delta$ extraídos dos dados da figura 6.4. A linha sólida e tracejada representam as predições $\phi=(2-\gamma) / 2(6.27)$ e $\delta=\gamma / 2$ (6.32). As incertezas são menores que o tamanho dos pontos. . . . . . .

6.6 (a) Tempo de sobrevivência $\tau$ vs. logaritmo do tamanho da população $\ln N$ no ponto crítico para $\sigma=2$ e vários valores de $\gamma$. (b) $\tau$ vs. $\ln N$ para $\gamma=1.5$ e diversos valores de $\sigma$. As linhas sólidas são ajustes da forma $\tau \sim[\ln N]^{\eta}$ (6.28). (c) Expoentes $\tau$ ajustados vs. $\gamma$. A linha sólida mostra a predição $\eta=2 /(2-\gamma)$ (6.29). As incertezas, se não explicitamente plotadas, são menores que o tamanho dos pontos. . . . . . . . . . . 78

6.7 (a) Densidade média $\langle\rho\rangle$ vs. tempo $t$ na fase ativa $\mu<\mu_{c}$ para $\gamma=1.5 \mathrm{e}$ diversos valores de $\mu$ (linhas sólidas). As linhas tracejadas representam $1.9 \times \rho_{\text {crit }}$, onde $\rho_{\text {crit }}=A t^{-\delta}$ é um ajuste da curva no ponto crítico $\mu=\mu_{c}$. Os círculos pretos são a estimativa do tempo de correlação $t_{x}$. (b) Densidade estacionária vs. distância ao ponto crítico $\left|\mu-\mu_{c}\right|$ para diversos valores de $\gamma$. (c) Tempo de correlação $t_{x}$ vs. $\left|\mu-\mu_{c}\right|$ para os mesmos valores de $\gamma$ encontrados no painel (b). As incertezas são da ordem da espessura das linhas ou do tamanho dos pontos.

6.8 Expoentes críticos $\beta$ e $\nu_{\|}$extraídos dos dados plotados nos painéis (b) e (c) da figura 6.7. As linhas sólidas indicam as predições $\beta=1$ e $\nu_{\|}=2 / \gamma$ (6.32). A incerteza em $\beta$ é da ordem de $10^{-2}$. . . . . . . . . . . .

6.9 Logaritmo do tempo de sobrevivência vs. $(\ln N)^{\gamma}$ de forma que o comportamento predito (6.37) seja visualmente linear. As linhas retas representam ajustes de $\ln \tau=C(\ln \tau)^{\gamma}+B$. O prefator $C$ está plotado na figura 6.10. As incertezas são menores do que o tamanho dos pontos. 
$6.10(\mathrm{a}, \mathrm{b})$ Prefator $C$ ajustado nos dados da figura 6.9 de acordo com a predição (6.37). A linha sólida representa um ajuste da lei de potência $C \operatorname{sim}|v|^{2-\gamma}$. (c) Expoente do prefator para diversos valores de $\gamma$. A linha sólida mostra a predição $2-\gamma(6.40)$. As incertezas são menores que o tamanho dos pontos se não plotadas. . . . . . . . . . . . . . . 82

6.11 Colapso dos tempo de sobrevivência de acordo com (6.39) para $\gamma=1.5$. Neste plote usamos uma desordem extremamente forte com parâmetros $\sigma=32$ e $\Delta t=10^{12}$. A correlação à escala $\tau_{0}(\mu)$ foi obtida ajustando a função $\tau \sim \tau_{0}(\mu) e^{C\left|\mu-\mu_{c}\right|^{2-\gamma}[\ln N]^{\gamma}}$ em cada uma das curvas. A tira cinza representa $\tau=D_{1} e^{D_{2} z}$, onde $D_{1}$ e $D_{2}$ são constantes com os valores

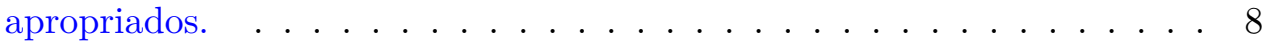

A.1 Exemplo de cadeia unidimensional com acoplamentos $J_{i}$ e campos $h_{i}$ associados a cada sítio $i \ldots \ldots \ldots \ldots \ldots$. . . . . . . . 86

A.2 Cadeia unidimensional após o tratamento perturbativo quando $h_{i}$ é muito maior que os outros termos na vizinhança de $i$. . . . . . . . . . . 88

A.3 Renormalização do campo $J_{i}$. . . . . . . . . . . . . . . . . . . 91 


\section{Lista de Tabelas}

2.1 Expoentes críticos para percolação direcionada $[9] \ldots \ldots$. . . . . . . . . 8

3.1 Tabela com as probabilidades de transição de uma interação para o algoritmo que define o modelo de contato e o algoritmo otimizado. $n_{I}$ representa o número de infectados na vizinhança de suscetíveis e $n_{S}$ o oposto. . . . . . . . . . . . . . . . . . . 18

4.1 Taxas de transição do modelo EQE. . . . . . . . . . . . . . . . 26

4.2 Estimativas de $\lambda_{c}(p)$. Para valores $p \geqslant 0.6$ foram usados $L=512 \mathrm{e}$ $L=1024$ ou $L=1024$ e 2048 caso contrário. . . . . . . . . . . . . . 35

4.3 Expoentes críticos estimados em $p=0.8$ e $\lambda=2.1075$ (1) para o processo de contato com diluição. A última coluna mostra os expoentes críticos para o modelo de Ising no campo aleatório transverso (RTFI Random Transverse Field Ising model). . . . . . . . . . . . . . . . . . 40

4.4 Expoentes críticos da classe de universalidade da percolação dinâmica. A tabela inclui os valores estimados através do modelo EQE e de outras referências. $\mathrm{O}$ valor de $\beta / \nu_{\perp}$ da referência [53], foi obtido usando a relação de escala $\beta / \nu_{\perp}=\left(d-2+\eta^{\prime}\right) / 2$, com $\eta^{\prime}=-0.04602(27)(7)$. Similarmente, usamos a relação $d-d_{f}=\beta / \nu_{\perp}$ para obter o valor de $\beta / \nu_{\perp}$, a partir dos valores numéricos encontrados nas referências $[55,54] . \ldots \ldots$. . . . 41

A.1 Operadores atuando nos autovetores de $H_{0} \ldots \ldots \ldots$. . . . . . 90

A.2 Operadores atuando nos autovetores de $H_{0} \ldots \ldots \ldots \ldots$ 


\section{Sumário}

1 Introdução

2 Classes de universalidade, teoria de escala de sistemas finitos e critério de Harris $\quad 3$

2.1 Percolação Dinâmica . . . . . . . . . . . . . . . . . . 3

2.2 Percolação Direcionada . . . . . . . . . . . . . . . . 7

2.3 Teoria de escala de sistemas finitos . . . . . . . . . . . . . 9

2.4 Estabilidade do ponto crítico na presença de desordem fraca . . . . . . 13

2.4.1 Critério de Harris . . . . . . . . . . . . . . . . . . 14

2.4.2 Generalização espaço-temporal do critério de Harris . . . . . . . 15

$\begin{array}{llr}3 & \text { Modelos } & 17\end{array}$

3.1 Processo de contato . . . . . . . . . . . . . . . . . 17

3.1.1 Desordem congelada fraca no espaço . . . . . . . . . . . . . 19

3.1.1.1 Correlações de longo alcance . . . . . . . . . . . . . . . . 20

3.1.2 Campo médio e a equação logística . . . . . . . . . . . . . . . 21

3.2 Modelo susceptível-exposto-infectado (SEI) . . . . . . . . . . . . . . 21

3.2 .1 Conexão com a percolação Isotrópica . . . . . . . . . . . . . . 23

4 Modelo epidemiológico de quatro estados $\mathbf{2 5}$

4.1 Definição do modelo epidemiológico de quatro estados . . . . . . . . . 25

4.2 Solução com aproximação de pares . . . . . . . . . . . . . 26

4.3 Simulações na rede quadrada . . . . . . . . . . . . . . . . . . . . . 29

4.3.1 Simulações dependentes do tempo . . . . . . . . . . . . . . 30

4.3.1.1 Simulações estacionárias . . . . . . . . . . . 34

4.3 .2 Ponto multicrítico . . . . . . . . . . . . . . . . 34

4.3.2.1 Expoentes críticos .............. 40 
4.4 Expoentes críticos da percolação dinâmica em $d=3 \ldots 4$. . . . . . . 40

5 Movimento Browniano fracionário refletido 43

5.1 Difusão normal . . . . . . . . . . . . . . . . . . . . . . . . . . 44

5.2 Movimento Browniano fracionário . . . . . . . . . . . . . . 45

5.3 Movimento Browniano fracionário com a parede refletora . . . . . . . 47

5.3 .1 Método . . . . . . . . . . . . . . . . . . . 47

5.3.2 Simulações na transição de regimes $v=0 \ldots \ldots$. . . . . . 48

5.3.3 Teoria dos regimes localizado $(v<0)$ e balístico $(v>0) \ldots \ldots 2$

5.3.4 Simulações nos regimes localizado $(v<0)$ e balístico $(v>0) \quad$. . 56

5.3 .5 Regime balístico $v>0 \ldots \ldots \ldots \ldots \ldots$

6 Equação logística com desordem temporal correlacionada $\quad 65$

6.1 Definição . . . . . . . . . . . . . . . . . . . . . 65

6.2 Mapeamento no movimento Browniano fracionário refletido . . . . . . . 66

6.3 Caso descorrelacionado $\gamma=\alpha=1 \ldots \ldots$. . . . . . . . . 67

6.4 Teoria da equação logística com desordem temporal correlacionada . . . 70

6.4 .1 Critério de Harris . . . . . . . . . . . . . . . . . . . . . . 70

6.4 .2 Comportamento crítico . . . . . . . . . . . . 71

6.4.3 Fase de Griffiths temporal . . . . . . . . . . . . . . . . . . 72

6.5 Simulações . . . . . . . . . . . . . . . . . . . . 73

6.5.1 Comportamento crítico . . . . . . . . . . . . . 74

6.5 .2 Fora do ponto crítico . . . . . . . . . . . . . 78

6.5.3 Fase de Griffiths temporal . . . . . . . . . . . . . . . . 80

7 Conclusão $\quad 83$

A Grupo de renormalização de desordem forte $\quad \mathbf{8 5}$

A.1 Solução do modelo de Ising aleatório no campo transverso unidimensional 86

A.1.1 Dizimação do campo $h_{i} \ldots \ldots \ldots \ldots$. . . . . . . 87

A.1.2 Dizimação do acoplamento $J_{i} \ldots \ldots \ldots \ldots$. . . . . . 88

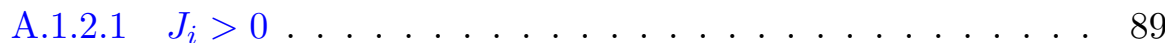

A.1.2.2 Caso $J_{i}<0 \ldots \ldots \ldots \ldots \ldots \ldots$

A.1.3 Equações de fluxo . . . . . . . . . . . . . . . . . . . . . 91

A.1.4 Renormalização dos comprimentos . . . . . . . . . . . . . 96

A.1.5 Renomalização do momento magnético . . . . . . . . . . . . . . 100

A.2 Comportamento crítico do processo de contato com desordem congelada 102 
A.2.1 Equação mestra do processo de contato com desordem congelada 103

A.2.2 Teoria de perturbação estocástica . . . . . . . . . . . . . . 105

A.2.2.1 Estados degenerados . . . . . . . . . . . . 107

A.2.3 Dizimação da taxa de recuperação . . . . . . . . . . . . . . . 107

A.2.4 Dizimação de um acoplamento . . . . . . . . . . . . . . . . . 109

A.2.5 Equações de fluxo e comportamento crítico . . . . . . . . . . . 111

B Renormalização da equação logística com desordem temporal $\mathbf{1 1 5}$

B.1 Equação logística com desordem temporal . . . . . . . . . . . . . . . . . 115

B.2 Renormalização . . . . . . . . . . . . . . . . . . . . . . 116

$\begin{array}{lr}\text { Referências Bibliográficas } & 121\end{array}$ 


\section{Capítulo 1}

\section{Introdução}

Transições de fase estão sempre presentes no cotidiano e podem ser observadas desde a evaporação, condensação e sublimação, até a condensação de Bose-Einstein e supercondutividade. Em modelos para a evolução de populações biológicas, transições de fase podem ser observadas entre a sobrevivência e extinção dessas populações. Do ponto de vista da mecânica estatística, a transição entre a sobrevivência e extinção corresponde a uma transição de fase de um estado ativo, com flutuações da população, para outro estado inativo, no qual as flutuações cessam e a população é extinta. Este tipo de transição de fase, comumente chamado de transição de fase absorvente, não obedece o balanceamento detalhado devido a existência de estados (absorventes) nos quais o sistema fica preso.

Modelos com transição de fase absorvente podem ser utilizados para a descrição de epidemias e dinâmica de populações [1], crescimento de interfaces [2], reações químicas [3], propagação de rachaduras [4], turbulência [5], entre outros [6, 7, 8, 9]. Sistemas sistemas experimentais com este tipo de transição incluem suspensões oscilando periodicamente [10, 11], a dinâmica de vórtices supercondutores [12] e biofilmes de colônias de bacterias $[13,14]$.

O estudo e classificação de transições de fase absorventes é um tópico ativo em mecânica estatística $[8,9,15]$, pois permite entender os mecanismos básicos por trás de cada transição. Do ponto de vista prático, por exemplo, em populações biológicas, o entendimento dos mecanismos básicos nos possibilita prever a extinção de populações biológicas e evitar a propagação de epidemias. Do ponto de vista teórico, este estudo nos permite classificar diferentes modelos com mecanismos similares em classes de universalidade. Por exemplo, consideremos a classe de universalidade onipresente em sistemas fora do equilíbrio com estados absorventes, a percolação direcionada. Segundo 
a conjectura de Janssen e Grassberger [16, 17], esta classe de universalidade abrange todos os modelos que apresentam uma transição de fase absorvente, parâmetro de ordem escalar e nenhuma simetria ou lei de conservação.

Nesta tese pretendemos ampliar o entendimento das transições de fase adicionando flutuações aleatórias no espaço (desordem espacial) e no tempo (desordem temporal). Iniciaremos a tese descrevendo um modelo epidemiológico com quatro estados. Apesar de não introduzirmos desordem neste modelo, veremos que ele é mapeado em um modelo epidemiológico com desordem congelada no espaço. Em seguida faremos o estudo do movimento Browniano fracionário refletido. Embora este problema não esteja diretamente relacionado com o tema desta tese, veremos que este movimento Browniano apresenta uma transição de fase e seu estudo será essencial para a compreensão do tópico seguinte. No capítulo 6 , faremos o estudo da equação logística com desordem temporal de longo alcance, através do mapeamento no movimento Browniano refletido e simulações de Monte Carlo. 


\section{Capítulo 2}

\section{Classes de universalidade, teoria de escala de sistemas finitos e critério de Harris}

Neste capítulo introduziremos a teoria básica que será utilizada no estudo das transições de fase no decorrer desta tese. Sabemos que as transições de fase podem ser classificadas em classes de universalidade de acordo com os mecanismos básicos de cada modelo, logo começaremos introduzindo duas classes de universalidade presentes em diversos modelos para a propagação de epidemias: a percolação dinâmica e a percolação direcionada. Em seguida, introduziremos o conceito básico de teoria de escala de sistemas finitos, conforme Kadanoff, que nos ajudará a compreender como as grandezas se escalam no ponto crítico.

\subsection{Percolação Dinâmica}

A classe de universalidade da percolação dinâmica (DyP) $[7,8,9]$ é caracterizada por gerar clusters mapeados nos clusters da percolação isotrópica [18]. Devido a este mapeamento, o estado estacionário de modelos nesta classe de universalidade contém infinitos estados absorventes (cada configuração de sítios ocupados na percolação isotrópica corresponde a um estado absorvente). Do ponto de vista epidemiológico, esta classe de universalidade pode ser relacionada a um processo de propagação de epidemia com imunização permanente. Neste caso, os sítios imunes correspondem aos sítios ocupados da percolação isotrópica. O comportamento qualitativo de modelos nessa classe de universalidade pode ser entendido como segue. Se a taxa de infecção for suficientemente 
Capítulo 2. Classes de universalidade, teoria de escala de sistemas finitos e critério de Harris

maior que taxa de imunização, a infecção irá se espalhar por toda a população. No estado estacionário, todos os indivíduos infectados tornam-se infectados. Esta é a fase ativa, ou, usando o linguajar da percolação, a fase percolante. Por outro lado, se a taxa de infecção for muito menor que a taxa de imunização, os indivíduos infectados rapidamente tornam-se imunes e a doença não se espalha. Esta é a fase inativa, ou a fase não percolante.

Entre a fase percolante e a não percolante, há uma transição de fase contínua na classe de universalidade da percolação dinâmica. Na transição de fase, grandezas como, por exemplo, o número de sítios ativos $N_{A}$, o raio médio $R$, o parâmetro de ordem (ou probabilidade de sobrevivência) $P$, comportam-se como leis de potência no tempo

$$
\begin{aligned}
& N_{A} \sim t^{\theta}, \\
& R \sim t^{1 / z},
\end{aligned}
$$

e

$$
P \sim t^{-\delta}
$$

Além do comportamento crítico temporal, o parâmetro de ordem $P$, o comprimento de correlação (espacial) $\xi$, e o comprimento de correlação temporal $\xi_{t}$, também comportamse como leis de potência no estado estacionário e próximo ao ponto crítico. Se $\epsilon$ é a distância ao ponto crítico, então

$$
\begin{gathered}
P \sim \epsilon^{\beta}, \\
\xi \sim \epsilon^{-\nu_{\perp}} .
\end{gathered}
$$

e

$$
\xi_{t} \sim \epsilon^{-\nu_{\|}}
$$

Os expoentes críticos $\theta, z, \delta, \beta, \nu_{\perp}$ e $\nu_{\|}$são expoentes característicos de todos os modelos pertencentes a esta classe de universalidade. Estes expoentes obedecem às relações

$$
\begin{aligned}
& \delta=\frac{\beta}{\nu_{\|}}, \\
& z=\frac{\nu_{\|}}{\nu_{\perp}},
\end{aligned}
$$

e

$$
\theta=\frac{d}{z}-2 \frac{\beta}{\nu_{\perp}}-1
$$


onde $d$ representa a dimensão espacial.

Nesta classe de universalidade, um modelo muito conhecido e estudado é o SuscetívelInfectado-Recuperado (SIR). As propriedades críticas desse modelo são estudadas nas referências [19, 20]. Neste modelo, cada sítio/indivíduo pode ser suscetível $(S)$, infectado $(I)$ ou recuperado $(R)$. A dinâmica deste modelo segue a sequencia $S \rightarrow I \rightarrow R$, sendo que a infecção de um sítio suscetível ocorre na presença de pares de sítios suscetívelinfectado, e sítios infectados recuperam-se espontaneamente. Se $N, 1-b$ e $t$ são o número de sítios na rede, a probabilidade de infecção, e o tempo, podemos simular o modelo SIR através do seguinte algoritmo:

1. Incrementamos $t$ por $1 / N$

2. Selecionamos um sítio aleatoriamente na rede

3. Caso o sítio seja $R$ voltamos para o passo 1 .

4. Se o sítio for $S$, haverá infecção com probabilidade $1-b$ vezes a fração de primeiros vizinhos infectados

5. Se não houver infecção, o sítio $I$ torna-se $R$ com probabilidade $b$.

Desse algoritmo, podemos perceber que a dinâmica termina quando não há sítios infectados, caso em que o modelo SIR alcança um estado absorvente com sítios recuperados e suscetíveis (todos os sítios infectados se tornam recuperados espontaneamente se $b>0)$. No estado absorvente, os clusters resultantes são mapeados nos clusters da percolação isotrópica.

Partindo da condição inicial na qual todos os sítios são suscetíveis, exceto por um sítio infectado no meio da rede, a dinâmica do modelo SIR gera apenas um cluster de sítios recuperados, que equivale a um cluster de sítios ocupados na percolação. A partir dessa condição inicial, descreveremos como medir as grandezas de interesse para este modelo.

Na percolação isotrópica, o parâmetro de ordem é a densidade de sítios no cluster percolante, ou seja, o número de sítios no cluster dividido pelo número total de sítios. No modelo SIR, podemos decidir se o sítio inicial pertence ao cluster percolante se a propagação da doença atinge uma das fronteiras da rede, isto é, se a infecção percola. Sendo assim

$$
P=\frac{\text { número de realizações percolantes }}{\text { número de realizações }} \text {. }
$$


Capítulo 2. Classes de universalidade, teoria de escala de sistemas finitos e critério de Harris

O quadrado do raio médio do cluster pode ser estimado a partir da expressão

$$
R^{2}=\frac{\left\langle\sum_{i \in \text { cluster }}\left|\overrightarrow{r_{i}}-\overrightarrow{r_{0}}\right|^{2}\right\rangle}{<N_{\text {cluster }}>},
$$

onde um sítio $i$ pertence ao cluster se o sítio estiver no estado recuperado e $N_{\text {cluter }}$ denota o número de sítios recuperados (ou número de sítios no cluster da percolação).

O número de sítios no cluster deve aumentar à medida que o número de sítios ativos $N_{A}$ aumenta, logo

$$
\frac{d}{d t} N_{\text {cluster }} \sim N_{A}
$$

Usando o comportamento crítico de $N_{A}(2.1)$, obtemos

$$
N_{\text {cluster }} \sim t^{\eta}
$$

com $\eta=1+\theta$. Também podemos calcular a fração de sítios pertencentes ao cluster no estado estacionário

$$
\rho=\frac{\left\langle N_{\text {cluster }}\right\rangle}{N} .
$$

Como $N_{\text {cluster }}$ é uma média em diferentes realizações, então

$$
\left\langle N_{\text {cluster }}\right\rangle=\frac{\sum_{\text {realizações }} \sum_{i \in \text { cluster }} 1}{\text { número de realizações }} .
$$

Combinando as equações (2.14) e (2.15), obtemos

$$
\rho=\frac{\sum_{\text {realizações }} \sum_{i \in \text { cluster }} 1}{N \times \text { número de realizações percolantes }} \times \underbrace{\frac{\text { número de realizações percolantes }}{\text { número de realizacões }}}_{\text {definição de } \mathrm{P}} .
$$

Perto do ponto crítico, o comprimento de correlação diverge e os clusters finitos tendem a ter propriedades similares ao percolante, sendo assim o primeiro termo da equação anterior será proporcional à $P$, que se escala com $\epsilon^{\beta}$, portanto

$$
\rho \sim \epsilon^{2 \beta}
$$

Analogamente, para o segundo momento de $\rho$

$$
\rho^{(2)}=<\rho^{2}>\sim \epsilon^{3 \beta} .
$$


Calculando a razão

$$
U=\frac{\rho^{(2)}}{\rho^{2}}
$$

perto do ponto crítico, obtemos

$$
U \sim \epsilon^{-\beta} .
$$

Sendo assim, a grandeza $U P[19]$ deve ser constante no ponto crítico.

\subsection{Percolação Direcionada}

A classe de universalidade da percolação direcionada (DP) [7, 8, 9, 21] é extremamente robusta e é uma das classes de universalidade mais comuns em modelos com estados absorventes. Transições de fase nessa classe de universalide podem ser encontradas em modelos para crescimento de interfaces [2], reações químicas catalíticas [3], propagação de rachaduras [4], entre outros.

Janssen e Grassberger formularam a conjectura-DP $[16,17]$ que traduzimos da referência [8] como: um modelo pertence à classe DP se

1. O parâmetro de ordem é escalar,

2. há uma transição de fase de uma fase ativa para outra absorvente e única,

3. as interações são de curto alcance,

4. não há atributos especiais tais como simetrias, leis de conservação ou aleatoriedade congelada.

O modelo mais comumente utilizado para introduzir esta classe de universalidade diz respeito à um líquido percolando numa rede em determinada direção. Considere uma rede na forma de pirâmide, conforme a figura 2.1. Nesta rede, as ligações entre os sítios podem estar abertas (sítios conectados) com probabilidade $p$, ou fechadas com probabilidade $1-p$. A cada instante de tempo, o líquido passa pelas ligações abertas e pode ocupar sítios na camada abaixo do sítio original. Note que o líquido não pode ir para cima, mesmo que haja uma ligação aberta.

Se as ligações ocorrerem com probabilidade $p$, há uma transição de fase contínua, em $p=p_{c} \approx 0.6446$, entre uma fase no qual o líquido não percola e outra no qual o líquido percola. Essa transição apresenta o comportamento crítico descrito por (2.1), (2.2), (2.3), (2.4), (2.5) e (2.6), sendo que o número de partículas ativas corresponde ao número de sítios ocupados na última iteração, e o parâmetro de ordem é a densidade 
Capítulo 2. Classes de universalidade, teoria de escala de sistemas finitos e critério de Harris

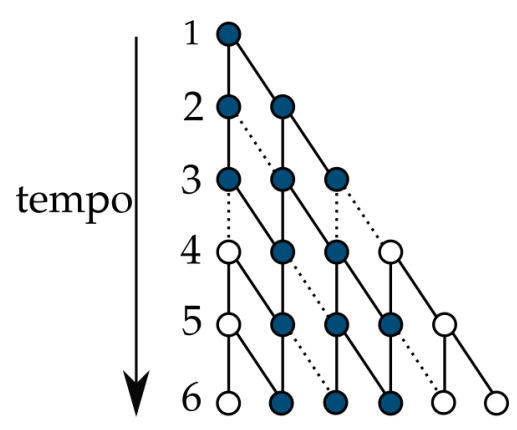

Figura 2.1: Exemplo da dinâmica DP num modelo onde o fluído está sob ação da gravidade e apenas pode cair. Os sítios azuis estão ocupados (contém líquido) e os brancos estão vazios. As linhas contínuas e tracejadas são as ligações abertas e fechadas, por onde o líquido pode ou não percolar.

Tabela 2.1: Expoentes críticos para percolação direcionada [9].

\begin{tabular}{|c|c|c|c|c|c|}
\hline $\mathrm{d}$ & $\beta$ & $\nu_{\perp}$ & $\nu_{\|}$ & $\theta$ & $\delta$ \\
\hline 1 & $0.27649(4)$ & $1.09684(6)$ & $1.73383(3)$ & $0.31368(4)$ & $0.15947(3)$ \\
\hline 2 & $0.583(4)$ & $0.733(4)$ & $1.295(6)$ & $0.2295(1)$ & $0.4505(10)$ \\
\hline 3 & $0.805(10)$ & $0.581(5)$ & $1.105(5)$ & $0.114(4)$ & $0.730(4)$ \\
\hline
\end{tabular}

de partículas ativas. No entanto, note que os expoentes críticos são diferentes daqueles encontrados na percolação dinâmica. Os expoentes críticos da classe de universalidade da percolação direcionada podem ser encontrados na tabela 2.1.

A dinâmica descrita para a propagação do líquido pode ser interpretada de duas maneiras. A primeira é como um modelo em duas dimensões, no qual o líquido percola direcionalmente numa rede $2 d$. A segunda é como um modelo em uma dimensão espacial e outra dimensão temporal, ou seja, um modelo em $1+1$ dimensões. Neste último caso, a dinâmica ocorre apenas em uma dimensão espacial, e a outra dimensão corresponde à história do processo. Para a interpretação em modelos em $1+1$ dimensões, as reações possíveis são de aniquilação, criação autocatalítica, e coalescência, conforme ilustrado na figura 2.2 .

Comparando as transições possíveis descritas no parágrafo acima, a dinâmica da

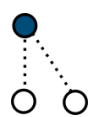

(a) aniquilação

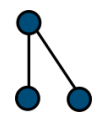

(b) criação autocatalítica

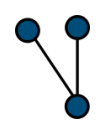

(c) coalescência

Figura 2.2: Transições DP em $1+1$ dimensões 


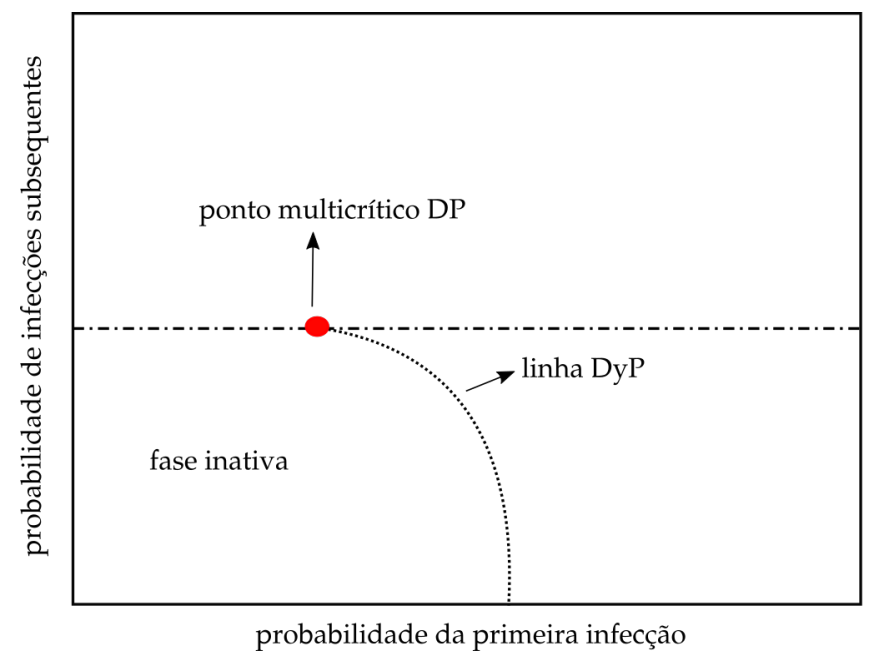

Figura 2.3: Esboço do diagrama de fase para um modelo semelhante ao SIR com reinfecção. O círculo vermelho corresponde a quando as taxas de infecção e reinfecção são idênticas.

percolação direcionada segue uma dinâmica muito parecida com a descrita pela percolação dinâmica. De fato, se permitirmos que os sítios recuperados sejam reinfectados no modelo SIR, a dinâmica é idêntica. De acordo com as referências [22, 23], o diagrama de fase para um modelo com probabilidade de reinfecção deve ser conforme a figura (2.3), apresentando uma linha de transição DyP e uma transição DP no ponto multicrítico.

Apesar das semelhanças entre DP e DyP, há diferenças sutis quando tratamos da criticalidade. Uma delas diz respeito ao cumulante universal no ponto crítico, dado por $[24]$

$$
u=\frac{<\rho^{2}>-<\rho>^{2}}{<\rho>^{2}},
$$

em contraste com o cumulante universal da percolação dinâmica $U P(2.19)$. Outra diferença remete aos expoentes $\eta$ e $\theta$, que são idênticos para a classe da percolação direcionada, pois os número de sítios no cluster é igual ao número de sítios ativos.

\subsection{Teoria de escala de sistemas finitos}

O comportamento crítico descrito até agora supõe que o sistema esteja no limite termodinâmico. Para os modelos descritos até agora o limite termodinâmico é tomado quando a rede tem tamanho infinito. Como é impossível simular sistemas infinitos, fazemos uso da teoria de escala de sistemas finitos. Essa teoria nos permite obter uma 
Capítulo 2. Classes de universalidade, teoria de escala de sistemas finitos e critério de Harris

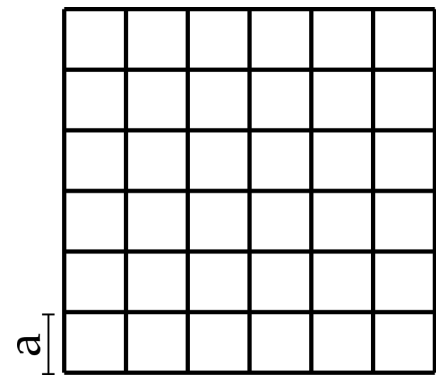

(a) Rede quadrada $6 \times 6$ com blocos de tamanho $a$

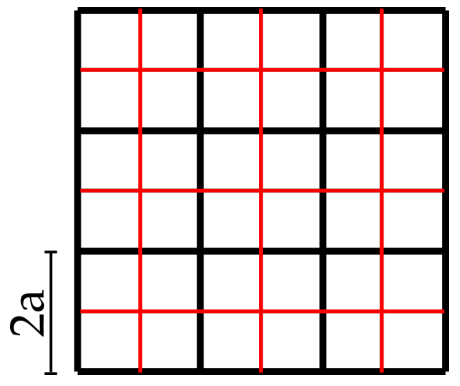

(b) Rede quadrada $3 \times 3 \mathrm{em}$ blocos de $2 \times 2$ com tamanho $2 a$

Figura 2.4: Exemplo da divisão em blocos de Kadanoff.

aproximação para as grandezas no limite termodinâmico. Introduziremos a teoria de escala de sistemas finitos seguindo as idéias contidas em [25].

Conforme proposto por Kadanoff, podemos dividir uma rede em blocos de tamanho $L$ conforme a figura (2.4). Se $\epsilon_{p}$ e $\epsilon_{h}$ são os parâmetros, Kadanoff propôs que a energia livre total deveria permanecer igual se reescalarmos os blocos. Se $f$ é a densidade de energia livre

$$
f\left(\epsilon_{p}, \epsilon_{h}\right)=L^{-d} f\left(\epsilon_{p_{L}}, \epsilon_{h_{L}}\right),
$$

com $\epsilon_{p_{L}}$ e $\epsilon_{h_{L}}$ dependendo de $L$ pois a alteração do tamanho dos blocos pode alterar as constantes de interação.

Além disso, o comprimento de correlação deve se escalar com o comprimento de correlação dos blocos multiplicado pelo tamanho do bloco

$$
\xi\left(\epsilon_{p}, \epsilon_{h}\right)=L \xi\left(\epsilon_{p_{L}}, \epsilon_{h_{L}}\right) .
$$

$\mathrm{Na}$ transição de fase, quando $\epsilon_{p} \rightarrow 0$ e $\epsilon_{h} \rightarrow 0$, a energia livre não é analítica e o comprimento de correlação diverge.

Derivando estas duas expressões com relação à $L$, obtemos o sistema de equações

$$
\left(\begin{array}{cc}
L^{-d} \frac{\partial f_{L}}{\partial \epsilon_{p_{L}}} & L^{-d} \frac{\partial f_{L}}{\partial \epsilon_{h_{L}}} \\
L \frac{\partial \xi_{L}}{\partial \epsilon_{p_{L}}} & L \frac{\partial \xi_{L}}{\partial \epsilon_{h_{L}}}
\end{array}\right)\left(\begin{array}{c}
\frac{d \epsilon_{p_{L}}}{d L} \\
\frac{d \epsilon_{h_{L}}}{d L}
\end{array}\right)=\left(\begin{array}{c}
d L^{-d-1} f_{L} \\
-\xi_{L}
\end{array}\right)
$$


no qual $f_{L}=f\left(\epsilon_{p_{L}}, \epsilon_{h_{L}}\right)$ e $\xi_{L}=\xi\left(\epsilon_{p_{L}}, \epsilon_{h_{L}}\right)$. Resolvendo este sistema obtemos

$$
\frac{d \epsilon_{p_{L}}}{d L}=\frac{1}{L} u\left(\epsilon_{p_{L}}, \epsilon_{h_{L}}\right)
$$

$$
\frac{d \epsilon_{h_{L}}}{d L}=\frac{1}{L} v\left(\epsilon_{p_{L}}, \epsilon_{h_{L}}\right),
$$

com

$$
u=\frac{f_{L} \frac{\partial \xi_{L}}{\partial \epsilon_{h_{L}}}+\xi_{L} \frac{\partial f_{L}}{\partial \epsilon_{h_{L}}}}{\frac{\partial f_{L}}{\partial \epsilon_{p_{L}}} \frac{\partial \xi_{L}}{\partial \epsilon_{h_{L}}}-\frac{\partial f_{L}}{\partial \epsilon_{h_{L}}} \frac{\partial \xi_{L}}{\partial \epsilon_{p_{L}}}}
$$

e

$$
v=-\frac{f_{L} \frac{\partial \xi_{L}}{\partial \epsilon_{p_{L}}}+\xi_{L} \frac{\partial f_{L}}{\partial \epsilon_{p_{L}}}}{\frac{\partial f_{L}}{\partial \epsilon_{p_{L}}} \frac{\partial \xi_{L}}{\partial \epsilon_{h_{L}}}-\frac{\partial f_{L}}{\partial \epsilon_{h_{L}}} \frac{\partial \xi_{L}}{\partial \epsilon_{p_{L}}} .}
$$

Assumiremos que $u$ e $v$ são funções analíticas, apesar de $f$ e $\xi$ não serem.

A fim de encontrarmos uma solução para estas equações, voltemos para eq. (2.23). Se $\epsilon_{p} \rightarrow 0$ e $\epsilon_{h} \rightarrow 0$, então $\xi$ diverge e, para qualquer $L$ finito, a única solução possível é $\epsilon_{p_{L}}=0$ e $\epsilon_{h_{L}}=0$. Sendo assim, $\epsilon_{p_{L}}=0$ e $\epsilon_{h_{L}}=0$ deve ser um ponto fixo das equações $(2.25)$ e (2.26). Portanto

$$
u(0,0)=0
$$

e

$$
v(0,0)=0 .
$$

Por outro lado, quando $L \rightarrow 0, \xi_{L} \rightarrow \infty$ e $\epsilon_{p_{L}}$ e $\epsilon_{h_{L}}$ tendem a zero. Ademais, as equações (2.25) e (2.26) não mudam pela substituição $L \rightarrow b L \forall b>0$, sugerindo que

$$
\epsilon_{p_{L}}=\phi(b L)
$$

e

$$
\epsilon_{h_{L}}=\psi(b L)
$$

também são soluções.

Expandindo (2.25) e (2.26) em torno de do ponto crítico $\left(\epsilon_{p}=\epsilon_{h}=0\right)$, ou equivalentemente quando $L<<1$, obtemos

$$
\frac{\partial \epsilon_{p_{L}}}{\partial L} \approx \frac{1}{L} \underbrace{\frac{\partial u(0,0)}{\partial \epsilon_{p_{L}}}}_{y} \epsilon_{p_{L}}
$$


Capítulo 2. Classes de universalidade, teoria de escala de sistemas finitos e critério de Harris

$\mathrm{e}$

$$
\frac{\partial \epsilon_{h_{L}}}{\partial L} \approx \frac{1}{L} \underbrace{\frac{\partial v(0,0)}{\partial \epsilon_{h_{L}}}}_{x} \epsilon_{h_{L}},
$$

cujas soluções são

$$
\epsilon_{p_{L}}=A L^{y}
$$

$$
\epsilon_{h_{L}}=B L^{x} .
$$

Dado que podemos renormalizar $L$ com uma constante $b$, a aproximação acima deve valer não apenas quando $L<<1$, mas para qualquer $L$, desde que exista um $b$ tal que $b L<<1$. Portanto podemos encontrar as constantes $A$ e $B$ substituindo $L=1$, obtendo as soluções para $\epsilon_{p_{L}}$ e $\epsilon_{h_{L}}$

$$
\epsilon_{p_{L}}=\epsilon_{p} L^{y}
$$

$\mathrm{e}$

$$
\epsilon_{h_{L}}=\epsilon_{h} L^{x} .
$$

Substituindo as equações acima em (2.23) com

$$
\begin{gathered}
y=\frac{1}{\nu_{\perp}}, \\
x=z,
\end{gathered}
$$

$\mathrm{e}$

$$
\epsilon_{h}=\frac{1}{t},
$$

obtemos

$$
\xi\left(\epsilon_{p}, t\right)=L \xi^{\prime}\left(\epsilon_{p} L^{1 / \nu_{\perp}}, t L^{-z}\right) .
$$

Próximo ao ponto crítico, $\xi^{\prime}$ deve assumir um valor constante, portanto

$$
\xi \sim L
$$

combinando as equações (2.37) e (2.38) com (2.43), desde que $\epsilon_{p_{L}}$ e $\epsilon_{h_{L}}$ convirjam com o aumento de $L$, temos

$$
\xi \sim \epsilon^{-\nu_{\perp}}
$$


$\mathrm{e}$

$$
\xi \sim t^{1 / z}
$$

que são as equações (2.5) e (2.2).

Nem todas as grandezas são como o comprimento de correlação $\xi$ e se escalam com $L^{1}$ conforme variamos o tamanho do bloco. Mais genericamente, podemos dizer que uma grandeza $Q$ se escalará com o expoente $-\mu / \nu_{\perp}$

$$
Q=L^{-\mu / \nu_{\perp}} Q\left(\epsilon_{p_{L}}, \epsilon_{h_{L}}\right) .
$$

Procedendo de forma análoga encontramos

$$
\begin{gathered}
Q=L^{-\mu / \nu_{\perp}} Q\left(\epsilon_{p} L^{1 / \nu_{\perp}}, t L^{-z}\right), \\
Q \sim \epsilon^{\mu}
\end{gathered}
$$

e

$$
Q \sim t^{-\mu / \nu_{\|}}
$$

Da mesma forma que relacionamos o parâmetro $\epsilon_{h}$ com o inverso do tempo, o parâmetro $\epsilon_{p}$ pode ser relacionado com uma medida de distância ao ponto crítico. Para o modelo de Ising, por exemplo, $\epsilon \sim T_{c}-T$.

\subsection{Estabilidade do ponto crítico na presença de desor- dem fraca}

Sistemas reais contém flutuações no espaço e seus parâmetros podem mudar no tempo. Podemos incorporar essas flutuações no modelo incluindo desordem fraca, isto é, uma desordem que tende a favorecer uma das fases em relação à outra, mas não quebra nenhuma das simetrias existentes. Este tipo de desordem pode ser obtida, por exemplo, mudando aleatoriamente os acoplamentos entre os sítios na rede.

O que ocorre com o comportamento crítico de uma transição de fase contínua na presença de desordem fraca? O ponto crítico permanece o mesmo ou a presença da desordem muda o comportamento crítico? A seguir introduziremos um critério que estabelece se o ponto crítico do sistema sem desordem, sistema limpo, permanece estável na presença de desordem congelada fraca. Primeiramente introduziremos o critério de Harris [26], que trata da estabilidade do comportamento crítico do sistema na presença de desordem congelada fraca no espaço. Em seguida, deduziremos uma generalização do 
Capítulo 2. Classes de universalidade, teoria de escala de sistemas finitos e critério de Harris

critério de Harris para desordem espaço-temporal, que pode ou não ser correlacionada.

\subsubsection{Critério de Harris}

Consideremos um bloco de comprimento de correlação $\xi$ e volume $\xi^{d}$ contendo sítios. Se $p$ for a probabilidade de um sítio estar afetado pela desordem, então o número médio de sítios com desordem será

$$
\langle n\rangle=\sum_{i} n_{i}=p \xi^{d}
$$

e a variância de $\langle n\rangle$ será

$$
\delta_{n}^{2}=p \xi^{d}(1-p)
$$

Dividindo a variância por $\xi^{2 d}$ obtemos a variância da concentração de desordem

$$
\delta_{p}=\frac{\sqrt{p \xi^{d}(1-p)}}{\xi^{d}} .
$$

Juntando as equações (2.5) e (2.52), é possível estimar a influência da flutuação da desordem perto do ponto crítico

$$
\delta_{p} \sim \epsilon^{d \nu_{\perp} / 2}
$$

A adição de desordem faz o ponto crítico ser uma função de $p$, portanto a distância ao ponto crítico será dada por uma curva $\epsilon^{\prime}(p)$. Entretanto, devido ao tamanho finito do bloco, haverá flutuações na concentração de impurezas no bloco de volume $\xi^{d}$ que fazem com que cada bloco tenha uma distância diferente do ponto crítico. Em vista disso, a distância do ponto crítico de cada bloco pode ser aproximada por

$$
\epsilon^{\prime} \approx \epsilon(p)+A \delta_{p}
$$

De acordo com (2.54), se as flutuações devido à desordem forem maiores que a distância global ao ponto crítico, isto é, $\delta_{p}>\epsilon$, ou ainda $d \nu_{\perp} / 2<1$, então a desordem deve ser relevante. Harris propôs em [26] que

$$
d \nu_{\perp}<2
$$

é uma condição necessária para que a desordem seja relevante. 


\subsubsection{Generalização espaço-temporal do critério de Harris}

Na referência [27], T. Vojta e R. Dickman propuseram a generalização do critério de Harris para o caso de desordem fraca espaço-temporal. A desordem espaço-temporal é introduzida fazendo que que a distância ao ponto crítico seja uma função da posição $x$ e do tempo $t$

$$
\epsilon^{\prime}=\epsilon+A n(x, t)
$$

no qual $A$ é uma constante, e $n(x, t)$ é uma variável de média (sobre a desordem) nula

$$
\langle n(x, t)\rangle=0
$$

e função de correlação invariante por translação

$$
\left\langle n(x, t) n\left(x^{\prime}, t^{\prime}\right)\right\rangle=G\left(x-x^{\prime}, t-t^{\prime}\right) .
$$

Na equação (2.56), o termo $\epsilon$ faz referência à distância ao ponto crítico do sistema sem desordem, enquanto que $n(x, t)$ representa o termo devido às flutuações da desordem. Assim como no caso do critério de Harris, a ideia da generalização espaço-temporal do critério de Harris é comparar ambos os termos a fim de decidir qual será o mais relevante.

Perto do ponto crítico, a flutuação média da distância ao ponto crítico devido à desordem pode ser avaliada através da variância de $n(x, t)$ sobre um volume definido pelos comprimentos de correlação espacial e temporal, ou seja, sobre um volume $V_{\xi}=$ $\xi^{d} \times \xi_{t}$ em $d+1$ dimensões

$$
\sigma^{2}\left(\xi, \xi_{t}\right)=\left\langle n(x, t) n\left(x^{\prime}, t^{\prime}\right)\right\rangle=\frac{1}{\xi^{2 d} \xi_{t}^{2}} \int_{V_{\xi}, V_{\xi^{\prime}}} d^{d} x d t d^{d} x^{\prime} d t^{\prime} G\left(x-x^{\prime}, t-t^{\prime}\right) .
$$

Usando a invariância translacional, podemos eliminar a integral no volume $V_{\xi^{\prime}}$

$$
\sigma^{2}\left(\xi, \xi_{t}\right)=\frac{1}{\xi^{d} \xi_{t}} \int_{V_{\xi}} d^{d} x d t G(x, t)
$$

Agora podemos comparar as flutuações da distância ao ponto crítico devido às flutuações da desordem, com a distância ao ponto crítico $\epsilon \sim \xi^{-1 / \nu_{\perp}}(2.5)$. Se $\sigma / \epsilon \rightarrow 0$ quando $\epsilon \rightarrow 0$, então as flutuações devido à desordem tornam-se irrelevantes conforme nos aproximamos do ponto crítico, logo a desordem não altera o comportamento crítico do sistema sem desordem. Do contrário, se $\sigma / \epsilon \rightarrow \infty$, as flutuações devido à desordem dominam o comportamento crítico. Dessa forma, o critério para estabilidade do ponto 
Capítulo 2. Classes de universalidade, teoria de escala de sistemas finitos e critério de Harris

crítico limpo (sem desordem) será

$$
\xi^{2 / \nu_{\perp}-d} \xi_{t}^{-1} \int_{V_{\xi}} d^{d} x d t G(x, t) \rightarrow 0
$$

no limite $\epsilon \rightarrow 0$ (ou $\xi \rightarrow \infty$ e $\xi_{t} \rightarrow \infty$ ).

Para o caso de desordem espacial descorrelacionada $(G \sim \delta(x))$, obtemos

$$
\xi^{2 / \nu_{\perp}-d} \rightarrow 0
$$

Portanto o comportamento crítico do sistema limpo será estável se $d \nu_{\perp}>2$, em concordância com o critério de Harris (2.55).

Consideremos agora desordem temporal descorrelacionada $(G \sim \delta(t))$. Neste caso, obtemos $\xi^{2 / \nu_{\perp}} \xi_{t} \rightarrow 0$. Usando a relação entre o comprimento de correlação espacial e temporal (2.2), o critério será

$$
z \nu_{\perp}>2
$$

em concordância com [28, 29].

Para o caso de desordem temporal correlacionada em lei de potência, $G \sim|t|^{-\gamma}$, o critério prevê a estabilidade do ponto crítico limpo se

$$
\gamma z \nu>2 .
$$




\section{Capítulo 3}

\section{Modelos}

\subsection{Processo de contato}

O processo de contato (CP - contact process) foi proposto por T. E. Harris (1974) [30], e é um dos modelos mais simples que prediz uma transição de fase contínua em uma dimensão. Este modelo pertence à classe de universalidade da percolação direcionada e tem uma interpretação epidemiológica no qual sítios podem estar infectados $(I)$ ou suscetíveis $(S)$.

Se $\lambda$ é a taxa de infecção, um sítio suscetível se torna infectado com taxa $n_{I} \lambda / k$, onde $n_{I}$ é o número de primeiros vizinhos infectados e $k$ o número de coordenação da rede. Os sítios no estado infectado se recuperam espontaneamente com taxa unitária. Se $\lambda$ for pequeno, o número de sítios infectados tende a zero devido à recuperação espontânea dos sítios infectados, e o sistema atinge o estado absorvente (pois não é possível que haja infecção sem sítios infectados) sem sítios infectados. Esta é a fase onde há extinção. Por outro lado, caso $\lambda$ for maior que um valor crítico $\lambda_{c}^{0}$, o sistema tem um outro estado estacionário ativo, cuja densidade de infectados é não nula. Esta é a fase onde a infecção sobrevive. Entre a extinção e sobrevivência da infecção, em $\lambda=\lambda_{c}$, há uma transição de fase contínua na classe de universalidade da percolação direcionada (seção 2.2).

Se $t, N$ e $p_{\lambda}=\lambda /(1+\lambda)$ são o tempo, o número de sítios, e a probabilidade de infecção, o processo de contato pode ser simulado com o seguinte algoritmo:

1. incrementamos o tempo por $1 / N$

2. selecionamos aleatoriamente um sítio no estado $\sigma_{i}$ e seguimos as seguintes regras

- se $\sigma_{i}$ for suscetível, $\sigma_{i}$ será infectado com probabilidade $p_{\lambda} n_{I} / k$ 
Tabela 3.1: Tabela com as probabilidades de transição de uma interação para o algoritmo que define o modelo de contato e o algoritmo otimizado. $n_{I}$ representa o número de infectados na vizinhança de suscetíveis e $n_{S}$ o oposto.

\begin{tabular}{|c|c|c|}
\hline & Definição & Otimizado \\
\hline$\Delta t$ & $1 / N$ & $1 / N_{I}$ \\
\hline Prob. Recuperação & $\frac{N_{I}}{N} \frac{1}{1+\lambda}$ & $\frac{1}{1+\lambda}$ \\
\hline Prob. Infecção & $\frac{N-N_{I}}{N} \frac{\lambda}{1+\lambda} \frac{n_{I}}{k}$ & $\frac{\lambda}{1+\lambda} \frac{n_{S}}{k}$ \\
\hline
\end{tabular}

- se $\sigma_{i}$ for infectado, $\sigma_{i}$ será suscetível com probabilidade $1-p_{\lambda}$

3. retornamos à regra 1

Apesar dessa ser a definição do modelo de contato, ele não é ótimo para simulação, pois por muitas vezes selecionamos sítios cujo estado não pode ser alterado. Portanto, usaremos um algoritmo otimizado que mantém uma lista dos sítios infectados. Se $N_{I}$ é o número de sítios infectados, o algoritmos otimizado (proposto por Dickman [31]) para a simulação do processo de contato será

1. incrementamos o tempo por $1 / N_{I}$

2. selecionamos um sítio no estado $\sigma_{i}$ aleatoriamente na lista de sítios infectados e tomamos uma das seguintes ações

- com probabilidade $p_{\lambda}$ selecionamos um dos vizinhos de $\sigma_{i}$ e o infectamos caso seja suscetível

- com probabilidade complementar $1-p_{\lambda}, \sigma_{i}$ torna-se suscetível

3. retornamos à regra 1

A conexão entre esses dois algoritmos pode ser entendida da seguinte forma. Considere a tabela 3.1 com os valores da probabilidade de infecção e recuperação em uma iteração de cada um dos dois algoritmos, no qual

$$
n_{I}=\frac{\sum_{(i, j)} \delta_{\sigma_{i}, S} \sum_{j} \delta_{\sigma_{j}, I}}{N-N_{I}}
$$

e

$$
n_{S}=\frac{\sum_{(i, j)} \delta_{\sigma_{i}, I} \sum_{j} \delta_{\sigma_{j}, S}}{N_{I}}
$$


são o número de infectados ao redor de suscetíveis, e vice versa. A notação $\sum_{(i, j)}$ significa somar sobre todos os valores de $i$ na vizinhança de $j$. No entanto, somar o número de sítios infectados ao redor de sítios suscetíveis é essencialmente contar o número de pares infectado-suscetível, que é idêntico ao número de pares suscetível-infectado, de forma que as equações (3.1) e (3.2) tem o mesmo numerador. Por conseguinte, $\left(N-N_{I}\right) n_{I}=N_{I} n_{R}$.

Agora consideraremos que, ao invés de tomar apenas uma iteração usando o algoritmo que define o processo de contato, tomaremos $N / N_{I}$ iterações, que é equivalente a multiplicar a segunda coluna da tabela 3.1 por $N / N_{I}$. Utilizando o argumento de equivalência do parágrafo anterior, é fácil de ver que o algoritmo otimizado é equivalente a tomar $N / N_{I}$ iterações do algoritmo que define o modelo de contato.

\subsubsection{Desordem congelada fraca no espaço}

Desordem congelada no espaço pode ser adicionada ao processo de contato, por exemplo, fazendo com que as taxas $\lambda$ variem aleatoriamente nas ligações entre os sítios, ou removendo os sítios da rede.

O critério de Harris [26] (seção 2.4.1) prediz que o comportamento crítico de uma transição de fase contínua deve ser estável na presença de desordem fraca se $d \nu_{\perp}>2$ (2.55). Usando o expoentes críticos da percolação direcionada 2.1, podemos ver que a desordem congelada fraca deve desestabilizar o comportamento crítico do processo de contato sem desordem em 1, 2 e 3 dimensões.

Hooybergs et al. mostraram, através do grupo de renormalização de desordem forte $[32,33,34,35]$, que o comportamento crítico do processo de contato unidimensional muda drasticamente no limite de desordem infinita [36, 37] (no entanto veja [38]). Usando a linguagem de grupo de renormalização, o novo comportamento crítico, nomeado ponto crítico de desordem infinita, é caracterizado pelo crescimento sem limites da desordem com o aumento da escala do sistema. No ponto crítico, observamos o crescimento logarítmico do comprimento de correlação $\xi$ com o tempo

$$
\xi \sim\left[\ln \frac{t}{t_{0}}\right]^{1 / \psi}
$$

ao invés da relação do crescimento em lei de potência $\xi \sim \xi_{t}^{1 / z}(2.2)$ observado no processo de contato sem desordem. Além do comprimento de correlação espacial, o número de sítios infectados

$$
N_{I} \sim\left[\ln \frac{t}{t_{0}}\right]^{\theta}
$$


e a probabilidade de sobrevivência

$$
P \sim\left[\ln \frac{t}{t_{0}}\right]^{-\delta}
$$

também se escalam logaritmicamente. Em uma dimensão, os expoentes críticos $\psi, \theta$ e $\delta$ tomam os valores (exatos) $1 / 2, \sqrt{5}-1$ e $(3-\sqrt{5}) / 2$. Os cálculos do deste grupo de renormalização estão descritos no apêndice A.

Surpreendentemente, o comportamento crítico de desordem infinita descrito acima também é observado no modelo de Ising no campo aleatório transverso [34, 35], ou seja, o processo de contato com desordem congelada no espaço está na mesma classe de universalidade do modelo de Ising no campo aleatório transverso. Este resultado é extremamente interessante, pois mostra que o estudo das propriedades críticas de sistemas de não-equilíbrio pode ser estendido para o caso quântico de equilíbrio.

A adição de desordem induz a formação regiões arbitrariamente grandes localmente na fase ativa (regiões raras), enquanto que o bulk do sistema está na fase inativa. A presença dessas regiões raras faz com que a dinâmica do sistema fique extremamente lenta se $\lambda_{c}^{0}<\lambda<\lambda_{c}$, onde $\lambda_{c}^{0}$ e $\lambda_{c}$ são os valores do ponto crítico do sistema sem e com desordem. Na fase de Griffiths a densidade decai como uma lei de potência

$$
\rho \sim t^{-1 / z^{\prime}}
$$

em contraste com o decaimento exponencial no caso sem desordem. O expoente $z^{\prime}$ não é universal e varia continuamente $\operatorname{com} \lambda$.

\subsubsection{Correlações de longo alcance}

Em [39], Vojta et al. estudaram os efeitos da desordem congelada fraca com correlações de longo alcance no processo de contato unidimensional. A desordem congelada fraca é introduzida na taxa de infecção $\lambda$, que segue a função de correlação de longo alcance

$$
G(i-j)=\left\langle\lambda_{i} \lambda_{j}\right\rangle-\left\langle\lambda_{i}\right\rangle\left\langle\lambda_{j}\right\rangle \sim|i-j|^{-\gamma},
$$

sendo que $\gamma$ está entre 0 e 1 , e o índices $i$ e $j$ representam a posição dos sítios.

Foi demostrado que, na presença dessas correlações de longo alcance nas taxas, o comprimento de correlação diverge de acordo com

$$
\rho \sim\left|\lambda-\lambda_{c}\right|^{-2 / \gamma}
$$


na vizinhança do ponto crítico. Note que o expoente do comprimento de correlação

$$
\nu_{\perp}=2 / \gamma
$$

depende do expoente de decaimento das correlações. Além disso, também foi demonstrado que a densidade de indivíduos decai mais devagar que uma lei de potência

$$
\rho \sim \exp \left\{\frac{1}{z^{\prime}} \ln \left(\frac{t}{t_{0}}\right)^{\gamma}\right\}
$$

na fase de Griffiths.

\subsubsection{Campo médio e a equação logística}

A probabilidade de encontrar um sítio no estado infectado no processo de contato é igual à densidade de sítios infectados $\rho$. Se $P_{I S}$ é a probabilidade de encontrar um par infectado-suscetível, $\rho$ obedece a equação

$$
\frac{d \rho}{d t}=\lambda P_{I S}-\mu \rho
$$

onde reescalamos o tempo $t \rightarrow k t / \mu$. Na equação (3.11), $\mu$ faz o papel da taxa recuperação de indivíduos infectados.

A teoria de campo médio nos permite aproximar a probabilidade de encontrar um par suscetível-infectado pela probabilidade de encontrar um sítio infectado e um sítio suscetível, isto é, $P_{I S}=\rho(1-\rho)$. Utilizando a aproximação de campo médio na equação (3.11), obtemos

$$
\frac{d \rho}{d t}=\lambda \rho(1-\rho)-\mu \rho,
$$

que é a equação logística [40].

A equação logística com desordem temporal será discutida no capítulo 6.

\subsection{Modelo susceptível-exposto-infectado (SEI)}

O modelo suscetível-exposto-infectado (SEI) [41] tem três estados: suscetível $S$, infectado $I$, e exposto $E$, e é uma versão assíncrona do modelo de Alexandrowicz [42]. Note, no entanto, que neste modelo o estado exposto equivale a um indivíduo imune que não transmite a doença.

Este modelo está na classe de universalidade da percolação dinâmica e difere de outros modelos nesta classe de universalidade por ser mapeado exatamente na percolação 


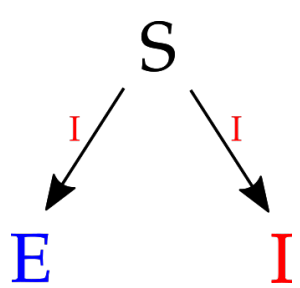

(a) Transições do modelo SEI

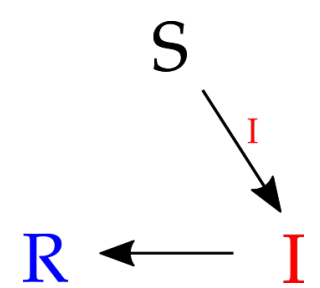

(b) Transições do modelo SIR

Figura 3.1: Transições possíveis do modelo SEI e SIR.

isotrópica. O mapeamento é dado interpretando os sítios infectados e expostos como os sítios ocupados e vazios da percolação isotrópica. Devido ao mapeamento exato, o modelo SEI apresenta a mesma probabilidade de transição da percolação isotrópica $\left(p_{c}=0.592746 \ldots[43]\right.$ em $\left.d=2\right)$, e, no estado estacionário, os sítios no estado $I$ e $E$ correspondem a sítios ocupados e vazios.

Definiremos a dinâmica do modelo SEI a seguir. Os sítios no estado $S$ se tornam $I$ ou $E$ na presença de sítios vizinhos no estado $I$ com probabilidade $p n_{i} / k$ e $(1-p) n_{i} / k$, onde $p$ é a probabilidade de infecção, $n_{i}$ é o número de sítios vizinhos no estado $I$ e $k$ é o número de coordenação da rede. A dinâmica do modelo SEI está ilustrada na figura 3.1. Para fins de comparação, também ilustramos as possíveis transições de outro modelo na classe de universalidade da percolação dinâmica, o modelo suscetívelinfectado-recuperado SIR (veja a definição do modelo SIR na seção 2.1). Diferente do modelo SIR cujo estado absorvente é composto apenas por sítios nos estados $S$ e $R$, os sítios no estado $I$ no modelo SEI não podem ter seu estado alterado, portanto o estado estacionário do modelo SEI é composto por infinitos estados absorventes com sítios nos estados $S, E$ e $I$, e sem pares $I S$.

Se $N$ for o número total de sítios, o modelo SEI pode ser simulado a partir do seguinte algoritmo

1. incrementamos o tempo por $1 / N$

2. selecionamos aleatoriamente um sítio no estado $\sigma_{i}$

- se $\sigma_{i}=I$ ou $\sigma_{i}=E$, voltamos para a regra 1

- caso contrário $\left(\sigma_{i}=S\right)$, o sítio selecionado se torna $I$ com probabilidade $p n_{i} / k$, ou $E$ com probabilidade $(1-p) n_{i} / k$

3. voltamos para a regra 1 
Assim como no caso do processo de contato, esse algoritmo não é ótimo para simulações, pois em muitos casos selecionamos sítios cujo estado não pode ser alterado. Sendo assim, usaremos um algoritmo otimizado. Se $N_{A}$ é o número de sítios ativos (sítios $S$ ao redor de cada $I$ )

1. incrementamos o tempo por $1 / N_{A}$

2. selecionamos um sítio aleatoriamente dentre os ativos, tornando-o infectado com probabilidade $p$, ou exposto caso contrário

3. retornamos para 1

É importante ressaltar que os sítios ativos correspondem aos sítios $S$ de cada par $I S$, portanto um sítio $S$ com $n$ vizinhos $I$ corresponde a $n$ sítios ativos. Note que cada sítio ativo corresponde a uma ligação ativa, isto é, uma ligação entre um sítio no estado $I$ e outro no estado $S$. Simulações do modelo SEI na rede quadrada podem ser encontradas em $[44,45]$.

\subsubsection{Conexão com a percolação Isotrópica}

Considere que cada sítio na rede está associado a uma variável aleatória $\zeta_{i}$. A variável $\zeta_{i}$ apresenta probabilidade constante no intervalo $[0,1)$. Na figura (3.2a), os sítios azuis e vermelhos representam sítios tal que $\zeta_{i}>p$ e $\zeta_{i}<p$ respectivamente. Sendo assim, os sítios vermelhos e azuis correspondem aos sítios ocupados e vazios da percolação isotrópica. A figura (3.2b) mostra o estado estacionário do modelo SEI com a condição inicial tal que o sítio central é infectado (vermelho) e todos os outros sítios são suscetíveis (branco), usando os mesmos valores de $\zeta_{i}$ para decidir se haverá infecção ou recuperação. Assim, podemos ver que a partir dessa condição inicial, o modelo SEI gera clusters isolados da percolação isotrópica com a mesma probabilidade de ocupação. 


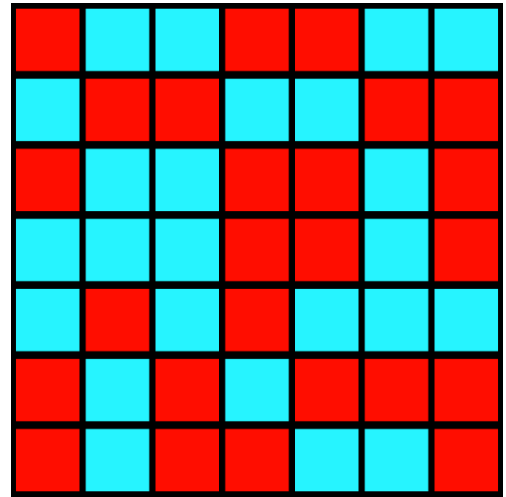

(a) Percolação isotrópica, sítios azuis e vermelhos são vazios e ocupados.

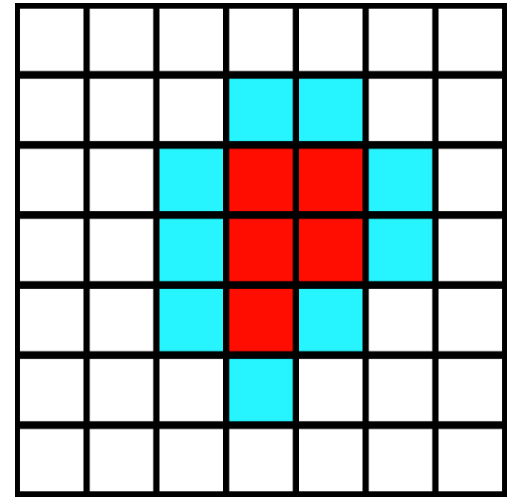

(b) Cluster gerado pelo modelo SEI no estado estacionário. Sítios azuis, vermelhos e brancos são expostos, infectados e suscetíveis.

Figura 3.2: Relação entre o estado estacionário do modelo SEI e a percolação. 


\section{Capítulo 4}

\section{Modelo epidemiológico de quatro estados}

Neste capítulo, introduziremos o modelo epidemiológico de quatro estados (EQE) [46]. Este modelo será resolvido por campo médio com aproximação de pares. Nesta solução, será possível identificar a relação deste modelo com o modelo suscetível-expostoinfectado (SEI) e o processo de contato (CP). Mais que isso, veremos a solução de campo médio indica que o estado estacionário do modelo EQE corresponde à dinâmica do CP no cluster gerado pelo do modelo SEI. Este resultado implicará que o modelo EQE terá as propriedades críticas do CP com desordem congelada.

\subsection{Definição do modelo epidemiológico de quatro estados}

No modelo EQE, um indivíduo pode ser suscetível $(S)$, imune $(U)$, exposto $(E)$ ou infectado $(I)$. Descreveremos a dinâmica desse modelo dividindo-o em duas etapas, a primeira onde indivíduos suscetíveis tornam-se imunes ou expostos a uma certa doença, e a segunda no qual a doença se espalha apenas entre os indivíduos expostos. Note que ambas as etapas ocorrem simultaneamente sem nenhuma preferência de ordem, no entanto essa divisão e ordenamento das etapas facilitará o entendimento das propriedades críticas modelo.

Na primeira etapa as transições são de exposição e imunização. No processo de exposição, indivíduos suscetíveis tornam-se expostos à doença na presença de outros seres expostos ou infectados. O processo de imunização é análogo, porém, ao invés do indivíduo inicialmente suscetível terminar no estado exposto, ele adquire imunidade à doença. Note que neste modelo um indivíduo exposto pode não passar para o estado 


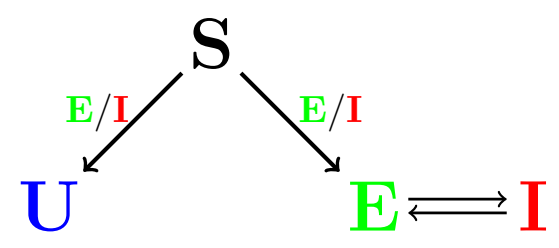

Figura 4.1: Reações do modelo EQE. Os estados são suscetível $S$, imune $U$, exposto $E$ e infectado $I$.

Tabela 4.1: Taxas de transição do modelo EQE.

\begin{tabular}{|c|c|}
\hline Transição & taxa \\
\hline$E+S \rightarrow E+U$ & $\alpha$ \\
\hline$E+S \rightarrow E+E$ & $\beta$ \\
\hline$I+S \rightarrow I+U$ & $\alpha$ \\
\hline$I+S \rightarrow I+E$ & $\beta$ \\
\hline$I+E \rightarrow I+I$ & $\lambda$ \\
\hline$I \rightarrow E$ & $r$ \\
\hline
\end{tabular}

infectado.

Após a primeira parte, há a formação de um cluster de indivíduos expostos e entramos na segunda parte da dinâmica do modelo EQE que ocorrerá neste cluster de indivíduos expostos. Nesta segunda parte, sítios expostos tornam-se infectados na presença de outros sítios infectados, e os sítios infectados podem retornar ao estado exposto espontaneamente. A figura 4.1 mostra as possíveis reações das duas etapas e a tabela 4.1 as taxas das transições.

\subsection{Solução com aproximação de pares}

Para resolver o modelo EQE, escreveremos as equações para a probabilidade $P_{X}$ e $P_{X Y}$ de encontrar sítios em um estado $X$, ou um par de sítios no estado $X Y$. Usando a 
tabela 4.1, as equações encontradas são

$$
\begin{aligned}
\frac{d P_{E}}{d t}= & \beta\left(P_{E S}+P_{I S}\right)-\lambda P_{I E}+r P_{I} \\
\frac{d P_{U}}{d t}= & \alpha\left(P_{E S}+P_{I S}\right) \\
\frac{d P_{E U}}{d t}= & \frac{\alpha}{k} P_{E S}+r P_{I U}-\mu \lambda P_{I E U}+\beta \mu\left(P_{E S U}+P_{I S U}\right)+\alpha \mu\left(P_{E S E}+P_{E S I}\right), \\
\frac{d P_{E S}}{d t}= & -\frac{(\alpha+\beta)}{k} P_{E S}-(\alpha+\beta) \mu\left(P_{E S E}+P_{E S I}\right)+ \\
& +\beta \mu\left(P_{I S S}+P_{E S S}\right)+r P_{I S}-\lambda \mu P_{I E S}, \\
\frac{d P_{U S}}{d t}= & \alpha \mu\left(P_{E S S}+P_{I S S}\right)-(\alpha+\beta) \mu\left(P_{U S E}+P_{U S I}\right) \\
\frac{d P_{I}}{d t}= & \lambda P_{I E}-r P_{I}, \\
\frac{d P_{I E}}{d t}= & \mu \lambda P_{I E E}+r P_{I I}-r P_{I E}-\frac{c}{k} P_{I E}-\mu \lambda P_{I E I}, \\
\frac{d P_{I U}}{d t}= & \mu \lambda P_{I E U}-r P_{I U}+\frac{\alpha}{k} P_{I S}+\alpha \mu\left(P_{I S E}+P_{I S I}\right) \\
\frac{d P_{I S}}{d t}= & -\frac{(\alpha+\beta)}{k} P_{I S}-(\alpha+\beta) \mu\left(P_{I S E}+P_{I S I}\right)-r P_{I S}+\lambda \mu P_{I E S},
\end{aligned}
$$

no qual $\mu=(k-1) / k$.

Somando as equações (4.1) com (4.6), (4.3) com (4.8), e (4.4) com (4.9), e definindo $W=E+I$, podemos reescrever as cinco primeiras equações desse conjunto

$$
\begin{aligned}
\frac{d P_{W}}{d t} & =\beta P_{W S}, \\
\frac{d P_{U}}{d t} & =\alpha P_{W S}, \\
\frac{d P_{W U}}{d t} & =\frac{\alpha}{k} P_{W S}+\beta \mu P_{W S U}+\alpha \mu P_{W S W}, \\
\frac{d P_{W S}}{d t} & =-\frac{\alpha+\beta}{k} P_{W S}-(\alpha+\beta) \mu P_{W S W}+\beta \mu P_{W S S}, \\
\frac{d P_{U S}}{d t} & =\alpha \mu P_{W S S}-(\alpha+\beta) \mu P_{U S W} .
\end{aligned}
$$

As equações acima podem ser identificadas com as equações para o modelo modelo SEI [41]. A solução no estado estacionário com aproximação de pares $\left(P_{X Y Z}=P_{X Y} P_{Y Z} / P_{Z}\right)$ 
das equações do modelo SEI é dada por

$$
\begin{aligned}
P_{W} & =p\left(1-s^{k}\right), \\
P_{U} & =1-P_{W}-s^{k}, \\
P_{W S} & =0, \\
P_{U S} & =q s^{k-1}\left(1-s^{k-1}\right), \\
P_{W U} & =p q\left(1-s^{k-1}\right),
\end{aligned}
$$

ou ainda

$$
\begin{aligned}
P_{I}+P_{E} & =p\left(1-s^{k}\right)=y_{0}, \\
P_{I S}+P_{E S} & =0, \\
P_{I U}+P_{E V} & =p q\left(1-s^{k-1}\right)=\omega_{0},
\end{aligned}
$$

sendo $s$ a raiz de

$$
p s^{k-1}-s+q=0
$$

$\operatorname{com} p=\beta /(\alpha+\beta)$ e $q=1-p$.

Com essa solução parcial, é possível verificar que as equações restantes (4.6), (4.7) e (4.8), fazem referência ao CP no cluster gerado pelo modelo SEI. Como o modelo SEI está na classe de universalidade da percolação dinâmica (com clusters diretamente mapeados na percolação isotrópica), essa aproximação sugere que o estado estacionário do modelo EQE está relacionado com o estado estacionário do $\mathrm{CP}$ nos clusters da percolação, ou seja, o CP em uma rede diluída ou com desordem congelada fraca.

Continuando a solução com a aproximação de pares, podemos levar em conta a condição (4.21) nas equações (4.7) e (4.8), obtendo

$$
\begin{aligned}
& \frac{d P_{I E}}{d t}=r P_{I I}-r P_{I E}-\frac{\lambda}{k} P_{I E}-\mu \lambda \frac{\left(P_{I E}\right)^{2}}{P_{E}}+\frac{\mu \lambda}{P_{E}} P_{I E} P_{E E} \\
& \frac{d P_{I U}}{d t}=-r P_{I U}+\frac{\mu \lambda}{P_{E}} P_{I E} P_{O U} .
\end{aligned}
$$

Substituindo $P_{I I}=P_{I}-P_{I E}-P_{I U}$ e $P_{E E}=P_{E}-P_{E U}-P_{I E}$, encontramos

$$
\frac{d P_{I E}}{d t}=r\left(P_{I}-2 P_{I E}-P_{I U}\right)-\frac{\lambda}{k} P_{I E}-\mu \lambda \frac{\left(P_{I E}\right)^{2}}{P_{E}}+\frac{\mu \lambda}{P_{E}} P_{I E}\left(P_{E}-P_{E U}-P_{I E}\right) .
$$

A partir das equações $(4.6),(4.25)$ e $(4.26)$, que contém as incógnitas $P_{I}, P_{I U}$ e 
$P_{I E}$, e das condições iniciais (4.20) e (4.22), analisaremos o comportamento crítico do modelo EQE com aproximação de pares. No estado estacionário, a partir de (4.6) e (4.25) temos

$$
\begin{aligned}
P_{I} & =\frac{\lambda}{r} P_{I E}, \\
P_{I U} & =\frac{\lambda \mu \omega_{0} P_{I E}}{y_{0} r+(\lambda \mu-\lambda) P_{I E}},
\end{aligned}
$$

que nos permite escrever (4.26) apenas em função de $P_{I E}$. Tomando o limite $P_{I E} \rightarrow 0^{+}$ encontramos a taxa critica da transição de fase do modelo EQE com aproximação de pares

$$
\frac{\lambda}{r}=\frac{1}{\mu} \frac{1}{1-\frac{\omega_{0}}{y_{0}}} .
$$

\subsection{Simulações na rede quadrada}

A solução de campo médio aponta que o modelo EQE deve apresentar o comportamento crítico do $\mathrm{CP}$ com diluição (DCP). Apesar disso, não é clara a conexão direta entre esses dois modelos, pois o DCP tem como parâmetros a probabilidade de ocupação $p$ e a taxa de infecção $\lambda$, enquanto que modelo EQE apresenta quatro taxas, e portanto necessita de três parâmetros (é possível fazer $r=1$, sem perda de generalidade). Contudo, escolhendo um parâmetro $e$ tal que

$$
\begin{gathered}
e p=\frac{\beta}{1+\alpha+\beta+\lambda}, \quad e(1-p)=\frac{\alpha}{1+\alpha+\beta+\lambda}, \\
(1-e) \frac{\lambda}{1+\lambda}=\frac{\lambda}{1+\alpha+\beta+\lambda} \quad \text { e } \quad(1-e) \frac{1}{1+\lambda}=\frac{1}{1+\alpha+\beta+\lambda},
\end{gathered}
$$

vemos que há um mapeamento direto entre o modelo EQE e o DCP, dado que o parâmetro $e$ determina a probabilidade com que simulamos cada uma das partes, isto é, simulamos o modelo SEI com probabilidade " $e$ " e o CP (nos clusters gerados pelo SEI) com probabilidade $1-e$. Com esta escolha de parâmetros concluímos que não deve haver transição de fase variando o parâmetro $e$, uma vez que não importa o quão rápido uma das dinâmicas seja com relação a outra no estado estacionário. A figura 4.2 mostra a dinâmica do modelo SEI e a do $\mathrm{CP}$, sendo que a figura 4.2a faz referência à dinâmica do SEI, e 4.2b ao CP. Note que ao combinar o sítio $E$ de ambas as dinâmicas chegamos no mesmo diagrama de transições do modelo EQE 4.1. 


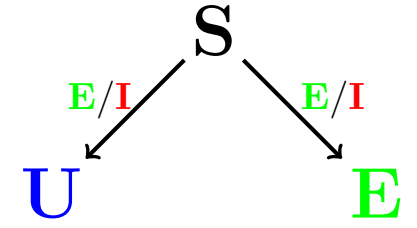

(a) Reações da primeira parte, relacionadas ao modelo SEI (geração do cluster).

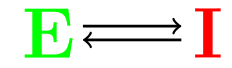

(b) Reações da segunda parte, relacionadas

ao processo

de contato no cluster.

Figura 4.2: Dinâmicas separadas do modelo de quatro estados. A primeira parte ocorre com probabilidade $e$, e a segunda com $1-e$.

\subsubsection{Simulações dependentes do tempo}

Conforme a seção anterior, o estado estacionário na fase ativa do modelo EQE corresponde ao processo de contato no cluster de sítios expostos. Como o processo de contato (e outros modelos com estados absorventes) necessita de meio infinito para a existência de um estado estacionário, a fase ativa do modelo EQE ocorrerá apenas na fase em que os sítios expostos percolam (cluster infinito), isto é, quando $p$ for maior que a probabilidade crítica de percolação $p_{c}$.

A fim de evitar efeitos de tamanho finito devido à geração de clusters, isto é, para evitar que a dinâmica dos sítios infectados desapareça antes da geração do cluster infinito de sítios expostos, as simulações serão feitas em $e=1$. Nesta situação, geraremos o cluster percolante de sítios expostos através do modelo SEI, e depois simularemos o CP nesse cluster. As simulações serão feitas com probabilidade de exposição $p=0.8$, para podermos comparar os valores com trabalhos já publicados.

Após a geração do cluster percolante de sítios expostos, seguiremos com a dinâmica do processo de contato. Partindo da condição inicial com um único infectado no centro do cluster de sítios expostos, procedemos até a chegada do estado absorvente ou se algum infectado encontrar a borda da rede. Com as curvas encontradas, estimaremos a taxa crítica pela menor taxa que apresentar um crescimento assintótico.

A figura 4.3 mostra os gráficos de $N_{I}$ (número médio de infectados) e $P$ (probabilidade de sobrevivência) em função do tempo em escala log-log. Os gráficos apresentados mostram as simulações para um tamanho de rede $L=8192$. Valores inferiores ( $L=1024$ e 4096) não se mostraram adequados para medir os expoentes críticos e determinar 

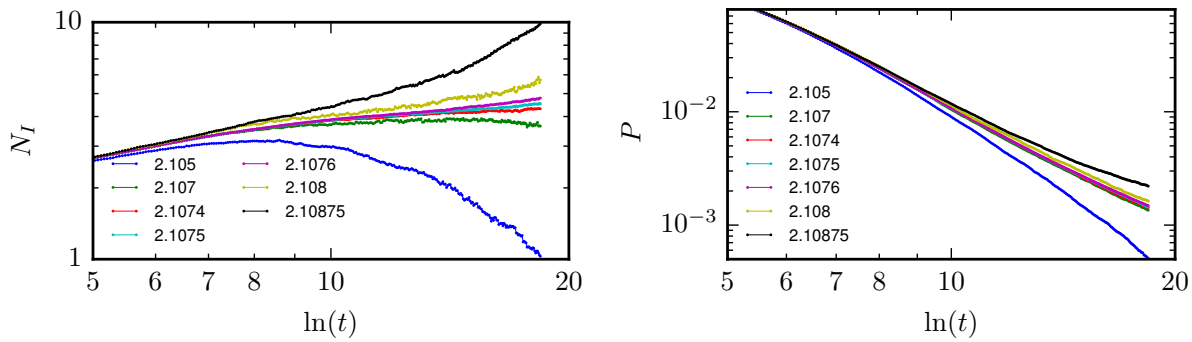

Figura 4.3: Simulações iniciadas a partir de uma única semente para $L=8192, p=0.8$ e $e=1$. As curvas são médias sobre um número de configurações de desordem que varia de $2 \times 10^{5}$ até $5 \times 10^{6}$. As incertezas são menores que $10 \%$.

com precisão a probabilidade de crítica de transição.

A taxa crítica de infecção, $\lambda_{c}$, será determinada através da curva de menor $\lambda$ com comportamento assintótico. No entanto, de acordo com os dados apresentados na figura (4.3), nos deparamos com um problema: para $N_{I}$, o valor crítico deve estar por volta de $\lambda=2.1075$, ao passo que para $P$, qualquer valor entre 2.105 e 2.10875 é uma estimativa para o valor crítico. A não concordância da estimativa do ponto crítico através dos gráficos de $N_{I}$ e $P$, provavelmente ocorre devido às fortes correções de tempo finito dadas pelo tempo microscópico $t_{0}$ no comportamento logarítmico que ocorre no ponto crítico:

$$
\begin{aligned}
& N_{I} \sim\left[\ln \left(t / t_{0}\right)\right]^{\Theta}, \\
& P \sim\left[\ln \left(t / t_{0}\right)\right]^{-\delta},
\end{aligned}
$$

e

$$
\xi \sim\left[\ln \left(t / t_{0}\right)\right]^{1 / \psi} .
$$

Podemos evitar o efeito de $t_{0}$ isolando $\ln \left(t / t_{0}\right)$ e descrevendo o comportamento crítico acima apenas em função de $N_{I}, P$ e $\xi$. Seguindo esse procedimento obtemos

$$
\begin{gathered}
N_{I} \sim P^{-\Theta / \delta}=P^{2\left(1-\nu_{\perp} / \beta\right)}, \\
N_{I} \sim \xi^{\Theta \psi}
\end{gathered}
$$

e

$$
P \sim \xi^{-\delta \psi} .
$$

As figuras $4.4,4.5$ e 4.6 mostram os gráficos de $N_{I}$ vs. $P, N_{I}$ vs. $\xi^{2}$, e $P$ vs. $\xi^{2}$, res- 


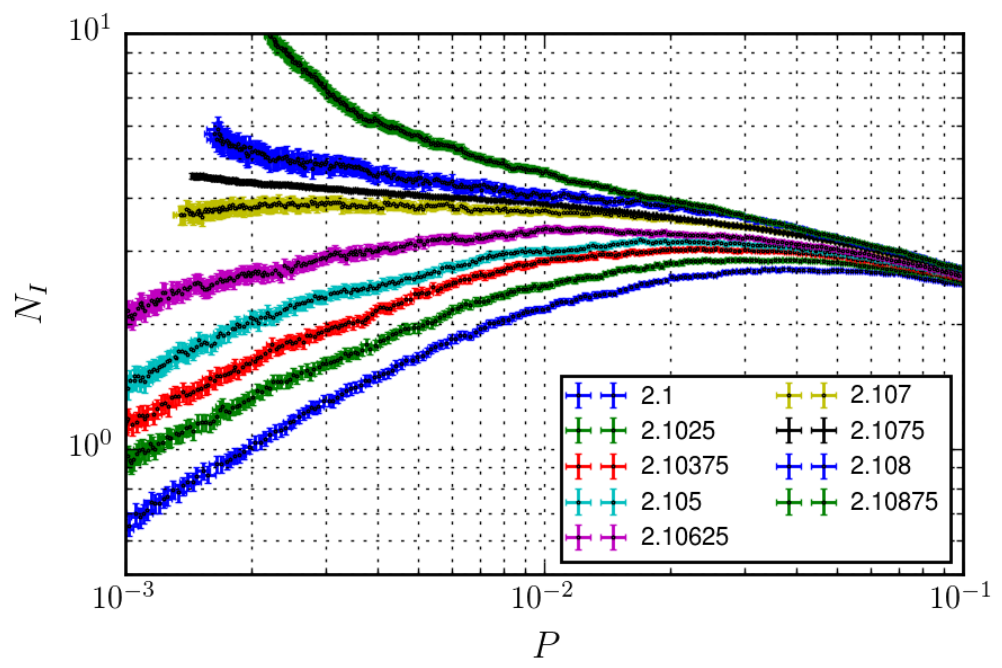

Figura 4.4: Gráfico de $P$ por $N_{I}$ para $L=8192$.

pectivamente. Desses gráficos podemos estimar o valor crítico $\lambda_{c}(p=0.8)=2.1075(1)$. Como este valor concorda com a estimativa realizada no gráfico de $N_{I}$ vs. $t$ (veja a figura 4.3), ajustamos a equação (4.31) nos dados de $N_{I}$ e obtemos $\Theta=0.145(25)$ e $\ln \left(t_{0}\right)=5.4(5)$.

Agora que obtemos a estimativa do valor crítico onde ocorre a transição de fase $\lambda_{c}(p=0.8)=2.1075(1)$ e o expoente crítico $\Theta$, estimaremos o valor dos outros expoentes críticos. Ajustando a equação (4.34) nos dados plotados na figura 4.4 encontramos $\beta / \nu_{\perp}=0.96(1)$. Similarmente, ajustamos a equação (4.35) nos dados plotados na figura 4.5, e, usando o valor $\Theta=0.145(25)$, obtemos $\psi=0.52$ (8). Por fim, encontramos $\delta=1.9(1)$ ajustando a equação (4.36) nos dados plotados na figura (4.6) e usando o valor estimado para $\psi$.

Após obter os expoentes críticos, estimaremos a linha da transição $\lambda=\lambda_{c}(p)$, a fim de compará-la com a estimativa obtida através da aproximação de pares (4.29). Para estimar esta linha da transição de fase, poderíamos aplicar o mesmo método usado para estimar o valor crítico de lambda em $p=0.8$, entretanto este método requer poder computacional do qual não dispomos, pois necessita que as simulações cheguem em tempos da ordem de $10^{8}$ para cada valor de $p$. Dessa forma, usaremos o método simplificado descrito a seguir. Ajustando uma lei de potência $t^{b}$ no intervalo de tempo $\left[10^{3}, 10^{5}\right]$ no ponto crítico $\lambda_{c}(0.8)=2.1075$ obtido para $p=0.8$, obtemos $b=0.06936$ (cuja incerteza será desprezada). Estimaremos o valor crítico $\lambda_{c}(p)$ como a média entre dois valores de $\lambda, \lambda_{\min }$ e $\lambda_{\max }$, tais que os valores de $b$ extraídos de ajustes nas mesmas 


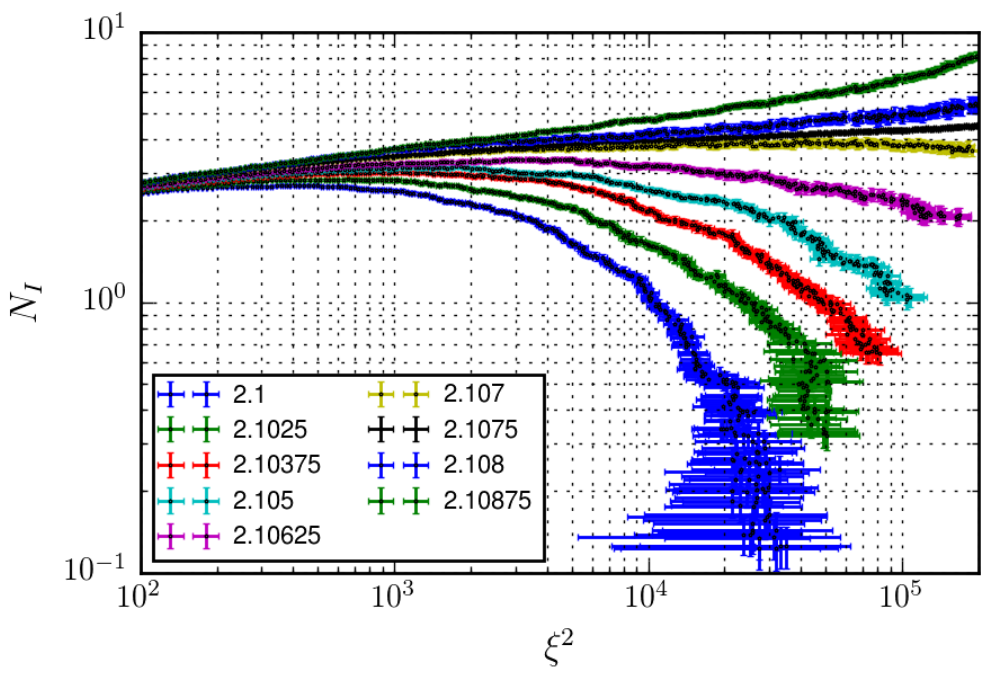

Figura 4.5: Gráfico de $\xi^{2}$ por $N_{I}$ para $L=8192$.

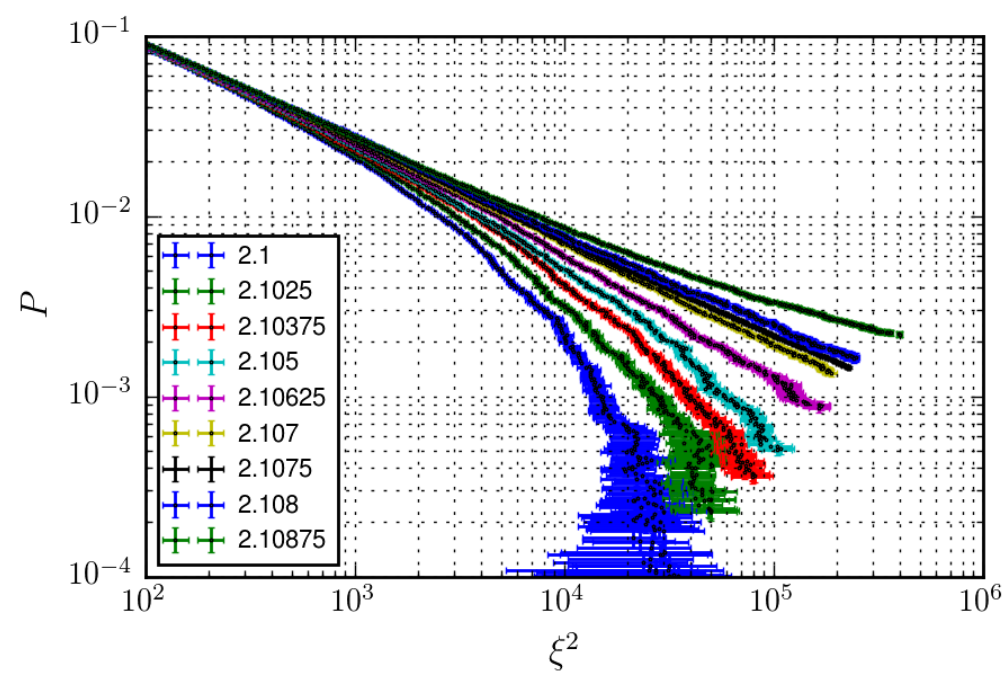

Figura 4.6: Gráfico de $\xi 2$ por $P$ para $L=8192$. 
condições acima, $b_{\min }$ e $b_{\max }$, obedecem $b_{\min }<b<b_{\max }$. Além disso, para evitarmos efeitos de tamanho finito, as estimativas de $\lambda_{c}(p)$ devem concordar entre tamanhos de rede $L$ e $2 L$. Apesar da carência de suporte teórico desse método, a tabela 4.2 mostra que os valores estimados são compatíveis com os calculados em outras referências. A figura 4.7 mostra os gráficos para $p=0.7$ e $p=0.98$, e 4.8 o diagrama de fase, com a linha crítica estimada pelo método acima e pela aproximação de pares (4.29).

\subsubsection{Simulações estacionárias}

Simulando em $p=0.8$ e em valores próximos e $\lambda_{c}(0.8)=2.1075$, estimaremos os valores dos expoentes críticos $\beta$ e $\nu_{\perp}$, definidos por

$$
\rho \sim\left(\lambda-\lambda_{c}\right)^{\beta}
$$

e

$$
\xi \sim\left(\lambda-\lambda_{c}\right)^{-\nu_{\perp}}
$$

onde $\rho$ é a densidade de sítios $N_{I}$ no cluster percolante $\left(N_{I} / N_{E}\right)$.

Para evitar que as simulações entrem no estado absorvente, começaremos da condição inicial no qual 1\% dos sítios expostos estão no estado infectado. Os gráficos na figura 4.9 mostram os resultados obtidos para as simulações com tamanho do sistema $L=2048$. Desses gráficos observamos que $\xi$ está longe do estado estacionário, enquanto que $\rho$ atinge o comportamento assintótico em $t \approx 10^{7}$. Tomando a média de $\rho$ no intervalo $\left[10^{7}, 10^{8}\right]$ obtemos $\beta=1.11(6)$, conforme (4.10). Usando a estimativa de $\beta$ e $\beta / \nu_{\perp}=$ $0.96(1)$, podemos encontrar $\nu_{\perp}=1.15(9)$.

\subsubsection{Ponto multicrítico}

O ponto multicrítico do processo de contato com diluição é o ponto da transição de fase na probabilidade crítica de percolação. Na referência [49] Hinrichsen at al. supuseram que no ponto multicrítico a densidade de infectados se comporta de acordo com

$$
\rho_{\mathrm{MC}} \sim\left(\ln \left(t / t_{0}\right)\right)^{-\bar{\delta}}
$$

$\mathrm{e}$

$$
\rho_{\mathrm{MC}} \sim\left(\lambda-\lambda_{c}\left(p_{c}\right)\right)^{\beta}
$$

Nesta mesma referência, os expoentes e o ponto crítico estimados através de simulações de Monte Carlo foram $\bar{\delta}=1.63(10), \beta=0.81(7)$ e $\lambda_{c}(0.5)=3.55(1)$. Nestas simulações, 
Tabela 4.2: Estimativas de $\lambda_{c}(p)$. Para valores $p \geqslant 0.6$ foram usados $L=512$ e $L=1024$ ou $L=1024$ e 2048 caso contrário.

\begin{tabular}{|c|c|c|c|}
\hline & este trabalho & $\operatorname{ref}[47]$ & ref [48] \\
\hline$p$ & $\lambda_{c}$ & $\lambda_{c}$ & $\lambda_{c}$ \\
\hline 0.5937 & $3.09(1)$ & - & - \\
\hline 0.5947 & $3.08(1)$ & - & - \\
\hline 0.5957 & $3.07(1)$ & - & - \\
\hline 0.5967 & $3.06(1)$ & - & - \\
\hline 0.5987 & $3.05(1)$ & - & - \\
\hline 0.60 & $3.04(1)$ & - & - \\
\hline 0.61 & $2.97(1)$ & - & - \\
\hline 0.62 & $2.90(1)$ & - & - \\
\hline 0.63 & $2.84(1)$ & - & - \\
\hline 0.64 & $2.78(1)$ & - & - \\
\hline 0.65 & $2.72(1)$ & - & - \\
\hline 0.66 & $2.66(1)$ & - & - \\
\hline 0.67 & $2.61(1)$ & - & - \\
\hline 0.68 & $2.56(1)$ & - & - \\
\hline 0.69 & $2.52(1)$ & - & - \\
\hline 0.70 & $2.47(1)$ & $2.4722(2)$ & $2.473(1)$ \\
\hline 0.71 & $2.43(1)$ & - & - \\
\hline 0.72 & $2.38(1)$ & - & - \\
\hline 0.73 & $2.34(1)$ & - & - \\
\hline 0.74 & $2.31(1)$ & - & - \\
\hline 0.75 & $2.27(1)$ & - & - \\
\hline 0.76 & $2.23(1)$ & - & - \\
\hline 0.77 & $2.20(1)$ & - & - \\
\hline 0.78 & $2.17(1)$ & - & - \\
\hline 0.79 & $2.13(1)$ & - & - \\
\hline 0.81 & $2.07(1)$ & - & - \\
\hline 0.82 & $2.04(1)$ & - & - \\
\hline 0.83 & $2.02(1)$ & - & - \\
\hline 0.84 & $1.99(1)$ & - & - \\
\hline 0.85 & $1.96(1)$ & - & - \\
\hline 0.86 & $1.94(1)$ & - & - \\
\hline 0.87 & $1.91(1)$ & - & - \\
\hline 0.88 & $1.89(1)$ & - & - \\
\hline 0.89 & $1.86(1)$ & - & - \\
\hline 0.9 & $1.845(1)$ & $1.8462(3)$ & $1.84640(5)$ \\
\hline 0.91 & $1.823(1)$ & - & - \\
\hline 0.92 & 1.801(1) & - & - \\
\hline 0.93 & $1.78(1)$ & - & - \\
\hline 0.94 & $1.76(1)$ & - & - \\
\hline 0.95 & $1.74(1)$ & - & $1.7410(1)$ \\
\hline 0.96 & $1.72(1)$ & - & - \\
\hline 0.97 & $1.701(1)$ & - & - \\
\hline 0.98 & $1.683(1)$ & - & $1.6844(1)$ \\
\hline 0.99 & $1.665(5)$ & - & - \\
\hline
\end{tabular}



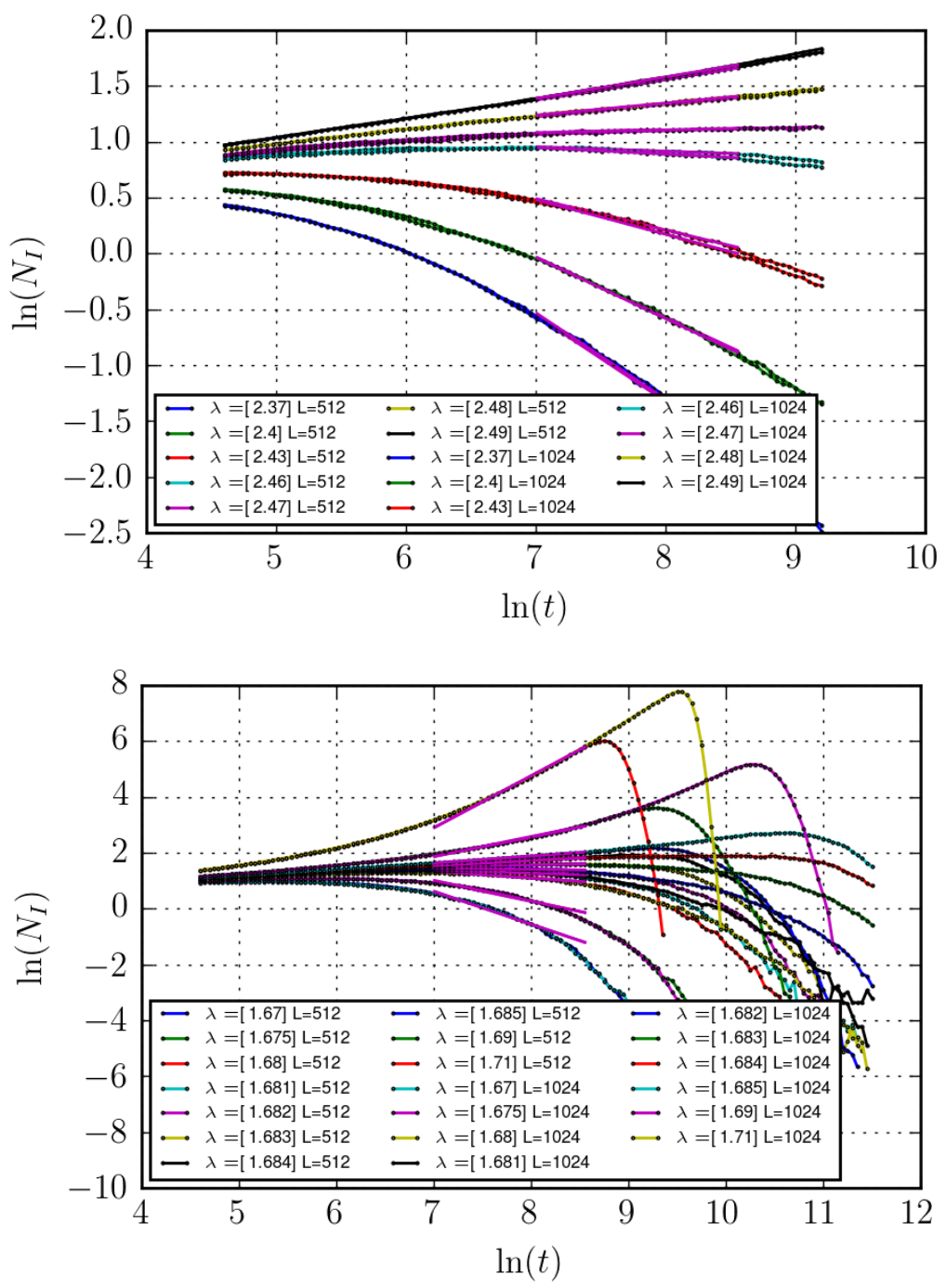

Figura 4.7: Ajuste de $t^{b}$ para $p=0.70$ e $p=0.98$. A linha rosa mostra o ajuste. 


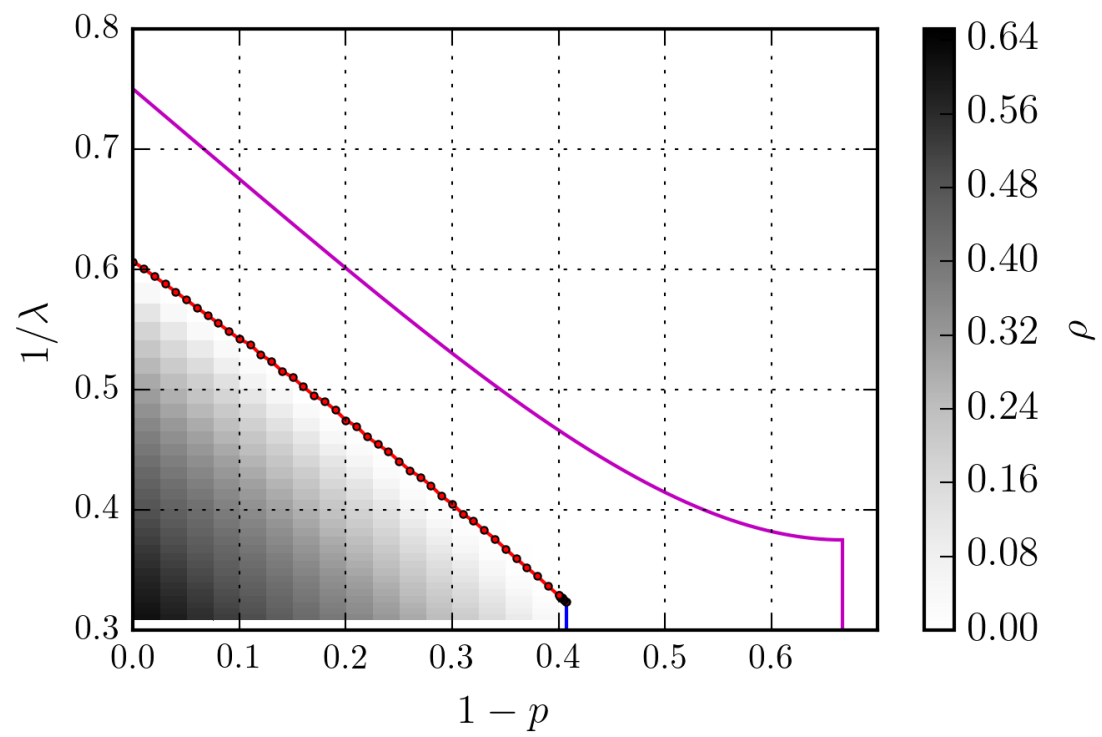

Figura 4.8: Diagrama de fase do DCP. Os pontos vermelhos indicam os valores de $\lambda_{c}(p)$, o degradê preto simulação estacionárias com $L=128$ e transiente de $10^{6}$, a linha vertical azul o ponto crítico da percolação $(0.59274 \ldots)$ e a linha rosa a aproximação de pares.

os autores usaram a percolação por ligação onde o ponto crítico de percolação $p_{c}=1 / 2$ é conhecido exatamente. Além disso, o tempo máximo de simulação foi $10^{6}$, o tamanho máximo do sistema foi $L=2000$ e foi fixado $t_{0}=1$.

Para reproduzir esses resultados, precisamos encontrar o valor crítico de $\lambda$ no ponto multicrítico, ou seja, o valor crítico de lambda no ponto crítico da percolação por sítio $(p=0.59274601 \ldots$ em $d=2)$. Para isso, extrapolamos linearmente os pontos da tabela 4.2 tais que $p<0.65$, estimando $\lambda_{c}\left(p_{c}\right)=3.10(1)$. Realizando simulações nesta estimativa do ponto multicrítico, obtemos $\bar{\delta}=2.6(2)$ para tempos da ordem de $10^{8}$, e tamanho do sistema $L=2048$. A figura (4.11) mostra as simulações no ponto multicrítico, onde a curva é uma média de cerca de 100 configurações de desordem, e condições iniciais com 100, 85, 50, 10 e $5 \%$ de sítios infectados em $t=0$. Nesta figura observamos que $\rho$ está claramente muito distante do estado estacionário não permitindo estimar $\beta$.

A discrepância entre os valores obtidos sugere que a dinâmica no ponto multi-crítico é extremamente lenta e estamos longe de encontrar características universais mesmo para tempos de $10^{8}$. 

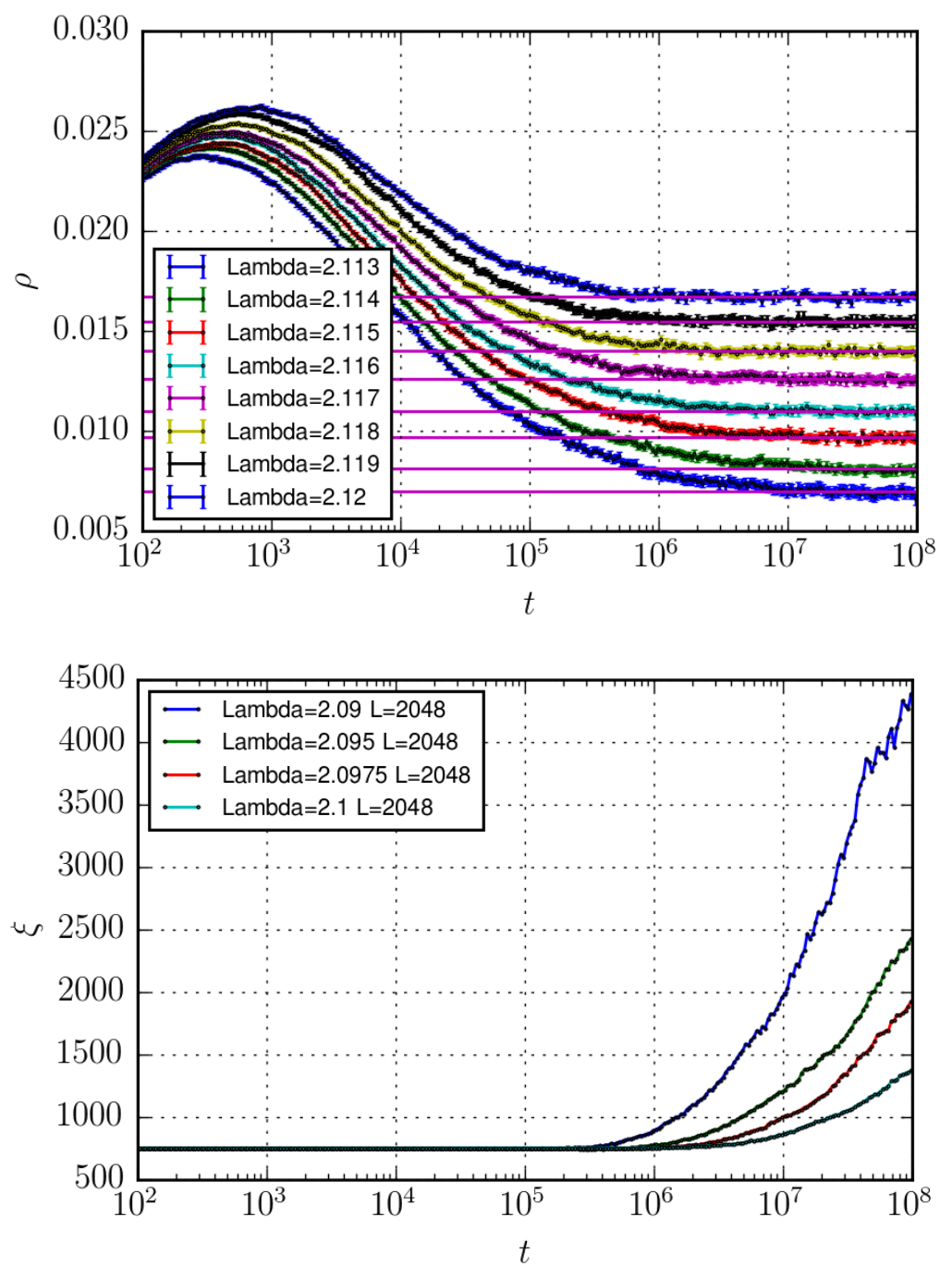

Figura 4.9: Simulações no estado estacionário de $\rho$ e $\xi$ vs. $t$, com tamanho do sistema $L=2048$. Cada curva é uma média de 20 até 40 configurações de desordem. A linha rosa no gráfico de $\rho$ mostra o valor adotado para as médias. 


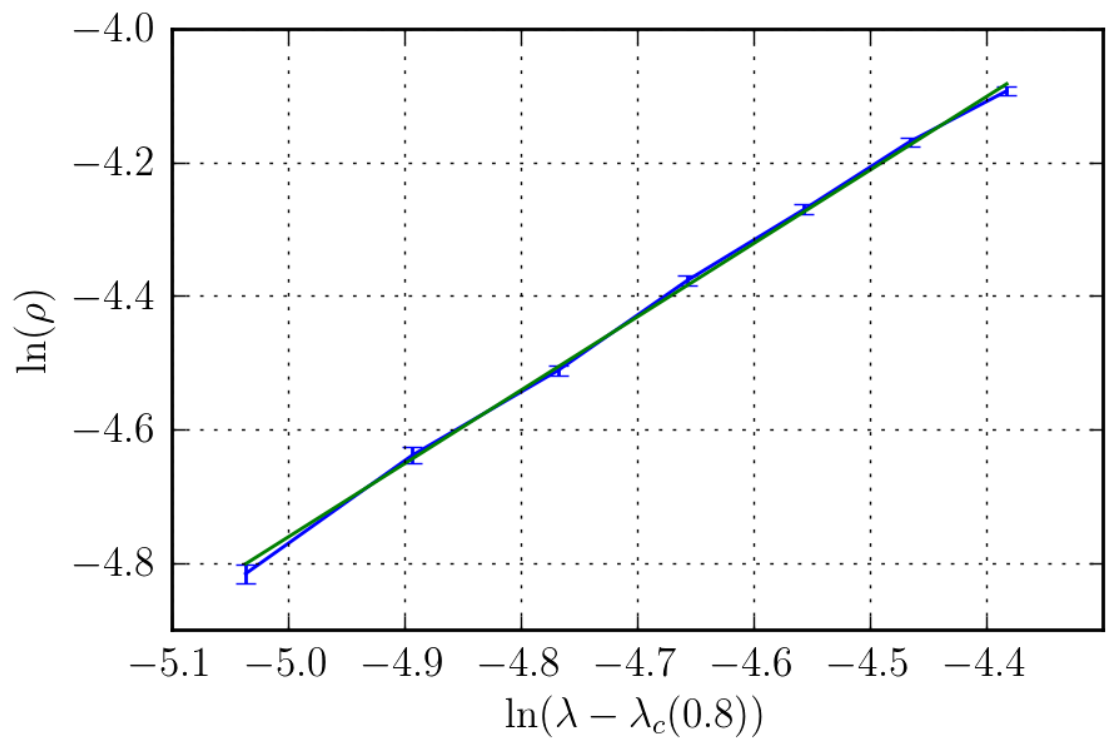

Figura 4.10: Ajuste de $\beta$ obtendo $\beta=1.11(6)$

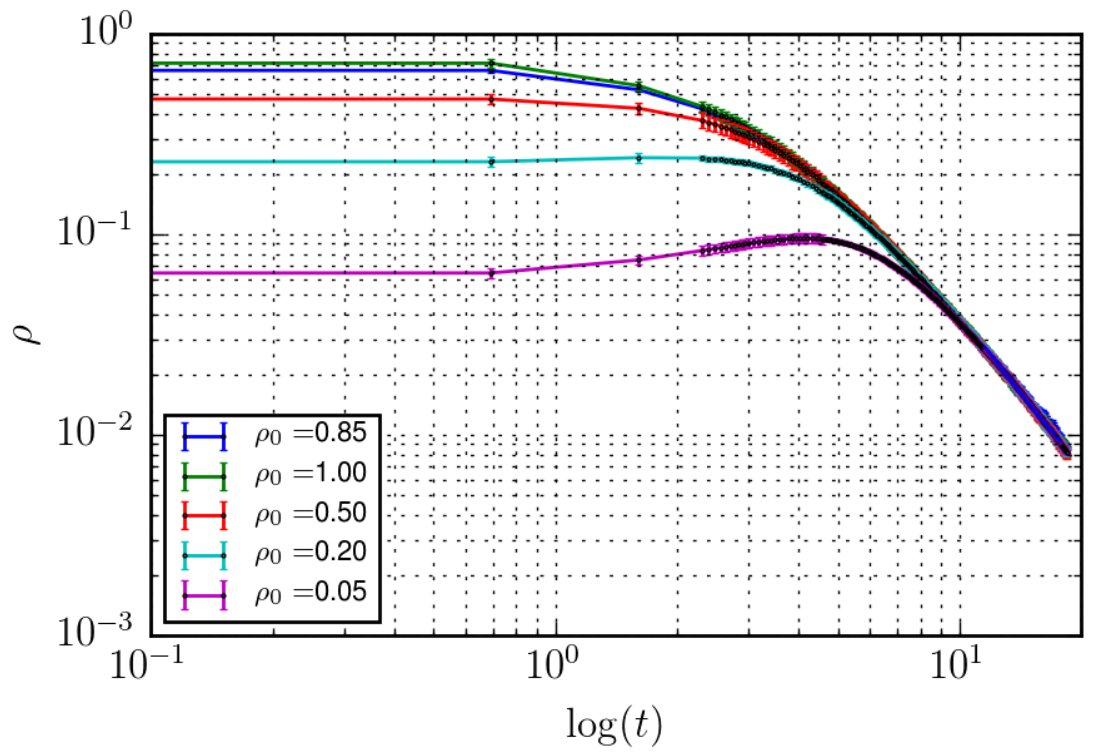

Figura 4.11: Simulações no ponto multi-crítico $\lambda_{c}\left(p_{c}\right)=3.10(1)$ com $L=2048$, tempos da ordem de $10^{8}$, cerca de 100 configurações de desordem e $\rho_{0}$ a densidade de sítios iniciais infectados. A linha azul indica o fit da função (4.39) com $t_{0}=1$ 
Tabela 4.3: Expoentes críticos estimados em $p=0.8$ e $\lambda=2.1075(1)$ para o processo de contato com diluição. A última coluna mostra os expoentes críticos para o modelo de Ising no campo aleatório transverso (RTFI Random Transverse Field Ising model).

\begin{tabular}{|c|c|c|c|c|}
\hline & este trabalho & ref $[47]$ & ref [48] & RTFI \\
\hline$\beta / \nu_{\perp}$ & $0.96(1)$ & $0.96(2)$ & $0.95(2)$ & \\
\hline$\beta$ & $1.11(6)$ & $1.15(9)$ & & \\
\hline$\nu_{\perp}$ & $1.15(9)$ & $1.20(15)$ & & $1.20(6)[50]$ \\
\hline$\Theta$ & $1.45(25)$ & $1.5(3)$ & & \\
\hline$\psi$ & $0.52(8)$ & $0.51(6)$ & $0.48(7)$ & $0.42(6)[51]$ \\
\hline$\delta$ & $1.9(1)$ & $1.9(2)$ & & \\
\hline
\end{tabular}

\subsubsection{Expoentes críticos}

Para finalizar este capítulo juntamos todos os expoentes críticos calculados na tabela 4.3 .

\subsection{Expoentes críticos da percolação dinâmica em $d=3$}

Como visto anteriormente, o modelo EQE pode ser visto como o modelo SEI e o CP acoplados (veja as figuras 4.1 e 4.2). Se $\lambda=0$ e $e=1$, isto é, se não há infecção e o modelo SEI é muito mais rápido que o $\mathrm{CP}$, o modelo EQE é reduzido ao modelo SEI, que apresenta a propriedades críticas na classe de universalidade da percolação dinâmica.

A figura 4.12 mostra as simulações do modelo EQE na rede cúbica, no caso que que o modelo EQE é reduzido ao modelo SEI. As simulações foram feiras no ponto crítico do modelo SEI, $p_{c}=0.31160768$ [52], que é o mesmo ponto crítico que o da percolação isotrópica. Desse gráfico podemos ver que as grandezas seguem leis de potência em mais de duas décadas no tempo, portanto efetuaremos ajustes em lei de potência no tempo, conforme o comportamento crítico esperado na classe de universalidade da percolação dinâmica (2.1), (2.4), (2.2) e (2.13), a fim de determinar os expoentes críticos. Os ajustes serão realizados para tamanhos do sistema $L=256,384,512,1024$ e 2048 .

Os gráficos na figura 4.13 mostram os expoentes obtidos em função de $L^{-1}$. Os expoentes $\theta, \eta$ e $\eta / \delta$ claramente apresentam uma variação sistemática com $L^{-1}$, logo estes expoentes precisam ser extrapolados. A extrapolação linear em $L^{-1}$ (linha vermelha) nos leva aos valores $\theta=1-\eta=0.4891(13)$ e $\eta / \delta=-4.283(5)$. Usando a relação de hiperescala (2.9), estimamos $\beta / \nu_{\perp}=0.47749$ (30) do valor de $\eta / \delta=4.283(5)$. Como todos os valores do expoente $z$ concordam dentro da incerteza, nossa estimativa de $z$ 

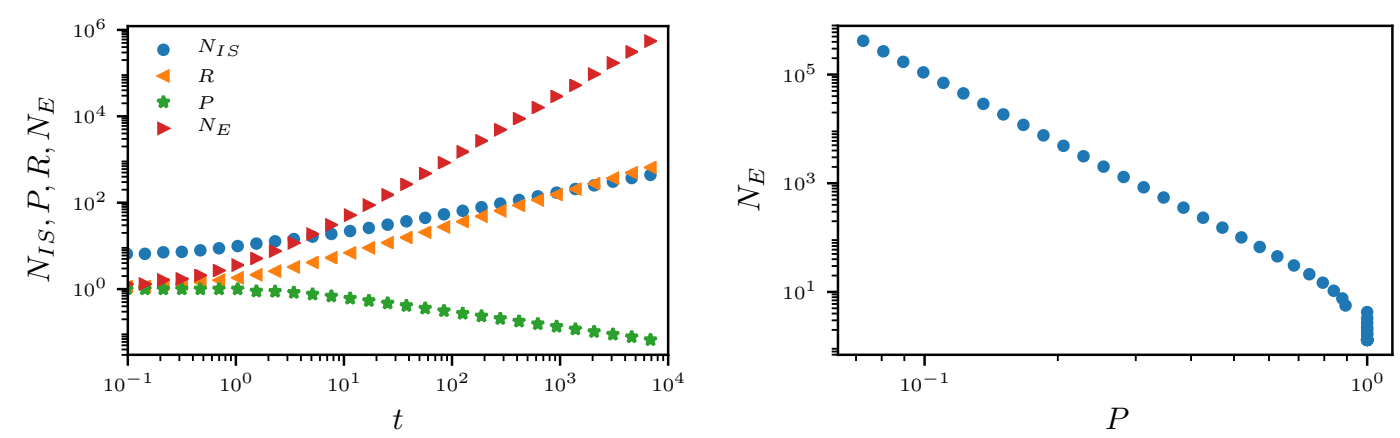

Figura 4.12: Gráfico do número de sítios ativos $N_{I}$, probabilidade de sobrevivência $P$, raio médio $R$, e do número de sítios expostos $N_{E}$. O tamanho do sistema é $L=2048$ e cada curve é uma média sobre $1.8 \times 10^{7}$ realizações. As incertezas são menores que o tamanho dos símbolos.

Tabela 4.4: Expoentes críticos da classe de universalidade da percolação dinâmica. A tabela inclui os valores estimados através do modelo EQE e de outras referências. $\mathrm{O}$ valor de $\beta / \nu_{\perp}$ da referência [53], foi obtido usando a relação de escala $\beta / \nu_{\perp}=\left(d-2+\eta^{\prime}\right) / 2$, $\operatorname{com} \eta^{\prime}=-0.04602(27)(7)$. Similarmente, usamos a relação $d-d_{f}=\beta / \nu_{\perp}$ para obter o valor de $\beta / \nu_{\perp}$, a partir dos valores numéricos encontrados nas referências [55, 54].

\begin{tabular}{ccccccc}
\hline & modelo EQE & {$[56]$} & {$[57]$} & {$[55]$} & {$[53]$} & {$[54]$} \\
\hline$\theta, \eta-1$ & $0.4891(13)$ & $0.488(7)$ & $0.494(6)$ & & & \\
$\delta$ & $0.3479(10)$ & $0.346(6)$ & $0.345(4)$ & & & \\
$z$ & $1.3737(5)$ & $1.375(5)$ & $1 / 0.728(2)$ & & & \\
$\beta / \nu_{\perp}$ & $0.47749(30)$ & & & $0.47707(10)$ & $0.477(3)$ & $0.47740(1)$ \\
\hline
\end{tabular}

será o valor mais preciso $z=1.3737(5)$. Por fim, os valores de $\delta$ parecem estar mudando com $L^{-1}$, porém não é claro como extrapolar estes dados no limite $L \rightarrow \infty$. Sendo assim, nossa estimativa será $\delta=0.3479(10)$, valor que corresponde ao maior tamanho da rede $(L=2048)$.

A tabela 4.4 mostra os expoentes críticos calculados através do modelo EQE junto com os valores calculados em outras referências. Comparando os expoentes críticos dinâmicos, $\theta, \eta, \delta$ e $z$, vemos que os valores calculados neste trabalho não só concordam com os valores das outras referências, mas também são mais precisos. Por outro lado, o expoente $\beta / \nu_{\perp}$ concorda com os valores encontrados nas referências [53, 54], mas não é compatível com o valor da referência [55 ${ }^{1}$.

\footnotetext{
${ }^{1}$ Note também que o valor de $\beta / \nu_{\perp}$ da referência [55] não é compatível com o da referência [54]
} 

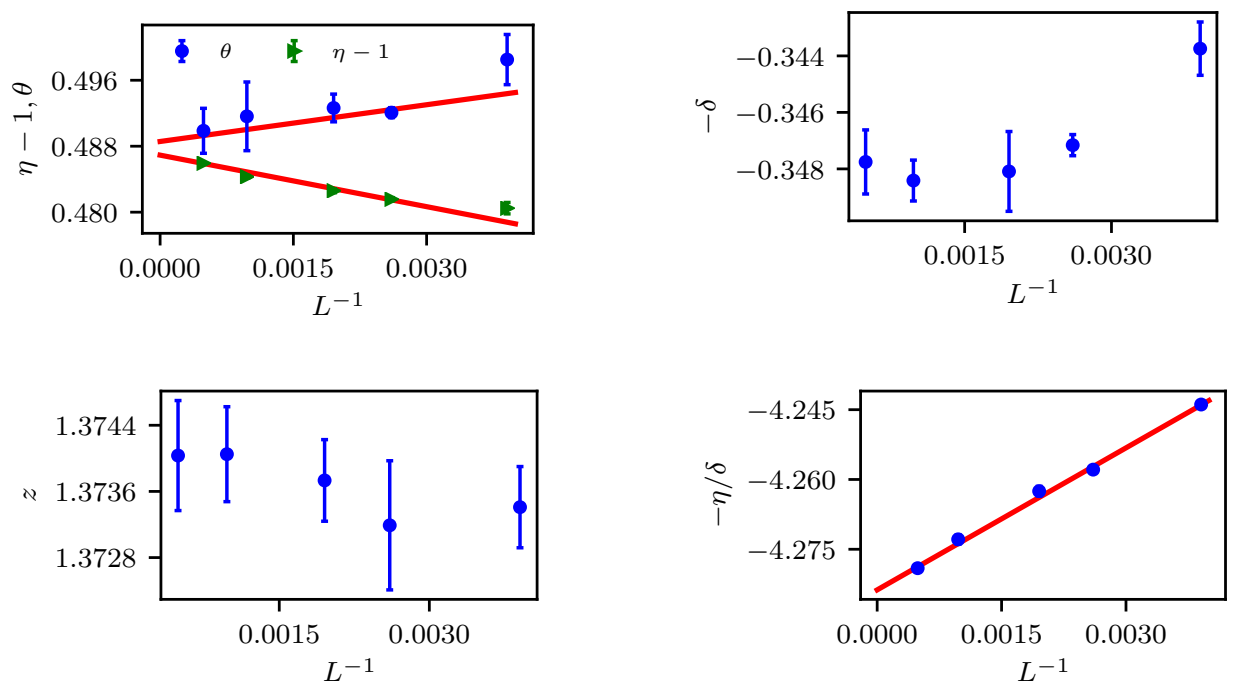

Figura 4.13: Expoentes críticos em função do inverso do tamanho do sistema $L^{-1}$. As linhas vermelhas são extrapolações lineares para $L \rightarrow \infty$. 


\section{Capítulo 5}

\section{Movimento Browniano fracionário refletido}

Embora o foco dessa tese não seja o estudo de passeios aleatórios e difusão, veremos no capítulo 6 que a equação logística, modelo de campo médio para a dinâmica da densidade indivíduos de uma população biológica, pode ser mapeada no movimento Browniano fracionário com uma parede refletora se permitimos que a taxas de crescimento e morte variem no tempo.

O estudo sistemático da difusão teve início no trabalho de Robert Brown em 1827 [58] e contínua como um tópico ativo de pesquisa [59, 60, 61, 62, 63, 64, 65].

Einstein, em seu trabalho pioneiro [66], mostrou que a difusão pode ser entendida como partículas que se deslocam aleatoriamente. Se a densidade de probabilidade deste deslocamento tiver variância e comprimento de correlação temporal finitos, então observamos difusão normal. A difusão normal é caracterizada pela variação linear da variância da posição das partículas com o tempo $\left\langle x^{2}\right\rangle-\langle x\rangle^{2} \sim t$. Caso essa condição não seja satisfeita, a difusão pode ser anômala. Atualmente, difusão anômala tem atraído atenção devido à observação do movimento de moléculas em sistemas complexos através das técnicas de microscopia moderna [67, 68, 69].

Um dos mecanismos que provoca difusão anômala é a presença de correlações temporais de longo alcance. O movimento Browniano fracionário [70, 71, 72, 73] é o modelo prototípico para um caminhante aleatório com correlações temporais de longo alcance, e tem aplicações na dinâmica de polímeros [74, 75], na dinâmica do mercado de ações [76], na difusão em células vivas [77], e tráfego de dados em redes [78]. Apesar da sua formulação simples, não há uma equação de difusão para o movimento Browniano fracionário, por isso é muito difícil encontrar a evolução da densidade de probabilidade 
da posição do caminhante aleatório com condições de contorno não triviais. No entanto, note que há estudos a respeito do problema da primeira passagem em um domínio semifinito [79, 80, 81, 82].

A seguir, faremos um estudo numérico do movimento Browniano fracionário na presença de uma parede refletora. Começaremos definindo os resultados já bem conhecidos para a difusão normal através da solução da equação de difusão. Posteriormente, definiremos a versão discreta do movimento Browniano fracionário (sem confinamento). Por fim, apresentaremos a teoria e as simulações do movimento Browniano fracionário com uma parede refletora em $x=0[83,84]$, e contrastaremos os efeitos da parede refletora com os resultados da difusão normal e do movimento Browniano fracionário não confinado.

\subsection{Difusão normal}

Considere uma partícula movendo-se no eixo $x$ e sujeita a um ruído aleatório descorrelacionado. Se esta partícula tiver velocidade média $v$, a densidade de probabilidade da posição $x$ dessa partícula no tempo $t, P(x, t)$, deve seguir a equação de difusão

$$
\frac{\partial P(x, t)}{\partial t}=\frac{\sigma^{2}}{2} \frac{\partial^{2} P(x, t)}{\partial x^{2}}-v \frac{\partial P(x, t)}{\partial x},
$$

onde $\sigma^{2} / 2$ é a constante de difusão. Se $x=0$ quando $t=0$, a solução da equação de difusão (5.1) será

$$
P(x, t)=\frac{1}{\sqrt{2 \pi \sigma^{2} t}} \exp \left\{-\frac{1}{2} \frac{(x-v t)^{2}}{\sigma^{2} t}\right\},
$$

ou seja, a densidade de probabilidade da partícula é uma gaussiana com média $\langle x\rangle=v t$ e variância $\left\langle x^{2}\right\rangle-\langle x\rangle^{2} \sim t$.

A parede refletora em $x=0$ pode ser inserida impondo a condição de inexistência de fluxo em $x=0, v P(0, t)-\left(\sigma^{2} / 2\right) \partial_{x} P(0, t)=0$. Sendo assim, a solução para o movimento browniano refletido será

$$
P_{\text {unc }}(x, t)=\frac{2}{\sqrt{2 \pi \sigma^{2} t}} e^{-\frac{1}{2} \frac{(x-v t)^{2}}{\sigma^{2} t}}-\frac{2 v}{\sigma^{2}} e^{\frac{2 v x}{\sigma^{2}}} \Phi\left(\frac{-x-v t}{\sigma^{2} t^{1 / 2}}\right),
$$

para $x \geq 0$, onde $\Phi$ é a distribuição gaussiana acumulada.

Três regimes podem ser distinguidas desta solução: o regime balístico $v>0$, quando a partícula caminha para longe da parede refletora; o regime localizado $v<0$, quando as partículas ficam presas próximas a parede refletora; e a transição entre os dois regimes 
$v=0$, quando há difusão normal $\left\langle x^{2}\right\rangle-\langle x\rangle^{2} \sim t$. Usando a linguagem de fenômenos críticos, $|v|$ assume o papel da distância ao ponto crítico.

No regime balístico, o caminhante aleatório desloca-se para longe da parede refletora e a densidade de probabilidade no limite de tempos longos tende a

$$
P(x, t)=\frac{1}{\sqrt{2 \pi \sigma^{2} t}} \exp \left\{-\frac{1}{2} \frac{(x-v t)^{2}}{\sigma^{2} t}\right\}
$$

assim como a densidade de probabilidade para o caso não confinado (5.2). No regime localizado, as partículas ficam presas perto da parede refletora e a densidade de probabilidade chega no estado estacionário

$$
P_{\mathrm{st}}=\frac{2|v|}{\sigma^{2}} \exp \left\{-\frac{2|v| x}{\sigma^{2}}\right\}
$$

Note que a posição média $\langle x\rangle$ é finita no regime localizado. Na transição entre os dois regimes $(v=0)$, a densidade de probabilidade é uma meia gaussiana

$$
P(x, t)=\frac{2}{\sqrt{2 \pi \sigma^{2} t}} \exp \left\{-\frac{x^{2}}{2 \sigma^{2} t}\right\}
$$

cuja variância se escala com $t$. Como a partícula é restrita a o eixo positivo de $x$, a posição média da partícula também se escala de acordo com $\langle x\rangle^{2} \sim t$.

A seguir, compararemos a densidade de probabilidade do movimento Browniano descorrelacionado sem e com a parede refletora. No regime balístico $(v>0)$, as duas densidades de probabilidade são similares, pois o caminhante aleatório viaja para longe da parede refletora. No regime localizado $(v=0)$, a parede refletora impede que o caminhante chegue à região negativa de $x$, fazendo com que haja um acúmulo de caminhantes na parede refletora. Na transição entre os regimes $(v=0)$, a parede refletora simplesmente corta o eixo negativo de $x$ e as duas densidades de probabilidade, dadas pelas equações (5.2) (5.6), são gaussianas.

\subsection{Movimento Browniano fracionário}

Definiremos o movimento Browniano fracionário discreto conforme Qian [85]. Seja $x_{n}$ a posição do caminhante aleatório no tempo discreto $n$. A posição do caminhante evolui de acordo com

$$
x_{n+1}=x_{n}+\xi_{n},
$$


onde $\xi_{n}$ é o ruído fracionário gaussiano, O ruído fracionário gaussiano é uma variável aleatória com correlações de longo alcance no tempo e densidade de probabilidade gaussiana de média $v$ e variância $\sigma$. A função de correlação de $\xi_{n}$ é dada por

$$
G(n)=\left\langle\xi_{m} \xi_{m+n}\right\rangle-\left\langle\xi_{m}\right\rangle\left\langle\xi_{m+n}\right\rangle=\frac{\sigma^{2}}{2}\left(|n+1|^{\alpha}-2|n|^{\alpha}+|n-1|^{\alpha}\right),
$$

$\operatorname{com} \alpha$, o expoente de difusão anômala, variando entre 0 e 2. A função de correlação acima decai como uma lei de potência no limite de tempos longos $n \rightarrow \infty$

$$
G \sim \alpha(\alpha-1) n^{\alpha-2}
$$

Do decaimento em lei de potência (5.9) podemos ver que o ruído fracionário gaussiano apresenta correlações positivas quando $\alpha>1$, correlações negativas quando $\alpha<1$, e é descorrelacionado quando $\alpha=1$.

Partindo de $x=0$ no instante inicial $t=0$, a função de correlação da posição do caminhante é dada por

$$
\left\langle x_{n} x_{m}\right\rangle-\left\langle x_{n}\right\rangle\left\langle x_{m}\right\rangle=\frac{\sigma^{2}}{2}\left(n^{\alpha}-|n-m|^{\alpha}+m^{\alpha}\right) .
$$

A variância da posição pode ser encontrada fazendo $n=m=t$. Nesse caso temos $\left\langle x_{t}^{2}\right\rangle-\left\langle x_{t}\right\rangle^{2}=\sigma^{2} t^{\alpha}$. Logo o movimento Browniano fracionário apresenta difusão anômala, sendo que o regime superdifusivo $\alpha>1$ está relacionado com as correlações positivas do ruído, enquanto que o regime subdifusivo $\alpha<1$ está relacionado com as correlações negativas.

A densidade de probabilidade da posição $x$ do movimento Browniano fracionário (não confinado) é gaussiana

$$
P_{n}(x)=\frac{1}{\sqrt{2 \pi \sigma^{2} n^{\alpha}}} \exp \left\{\frac{1}{2} \frac{(x-v n)^{2}}{\sigma^{2} n^{\alpha}}\right\} .
$$

Se $\alpha=1$, a distribuição de probabilidades (5.11) se reduz ao caso descorrelacionado (5.2). 


\subsection{Movimento Browniano fracionário com a parede refle- tora}

\subsubsection{Método}

Após apresentarmos os resultados da difusão normal e do movimento Browniano fracionário não refletido, voltamos a nossa atenção para o movimento Browniano fracionário refletido.

A parede refletora localizada em $x=0$ pode ser implementada de diversas formas, por exemplo, modificando a equação (5.7) na forma

$$
x_{n+1}=\left|x_{n}+\xi_{n}\right|,
$$

$\mathrm{ou}$

$$
x_{n+1}=\left\{\begin{array}{ll}
x_{n}+\xi_{n}, & \text { se } x_{n}+\xi_{n}>0 \\
x_{n}, & \text { caso contrário }
\end{array} .\right.
$$

Ambas as implementações resultam no mesmo comportamento assintótico no regime balístico $(v>0)$ e na transição dos regimes $(v=0)$, pois a densidade de probabilidade da posição $x$ alarga com o tempo. No regime balístico $(v<0)$, a densidade de probabilidade fica concentrada perto da parede refletora, fazendo com que $x$ seja da ordem do tamanho típico do ruído $\xi_{n}$, logo diferentes implementações da parede refletora podem resultar em diferentes correções devido ao tempo discreto da implementação. Este tópico será discutido na seção 5.3.4, onde mostraremos as simulações no regime localizado.

Para gerar os números aleatórios com densidade de probabilidade gaussiana e função de correlação (5.8), usaremos o método de filtração de Fourier [86], que descreveremos a seguir. Primeiramente geramos números aleatórios gaussianos, descorrelacionados, com média nula e variância unitária, $\nu_{n}$. Em seguida, calculamos $\tilde{\nu}_{n}$ e $\tilde{G}_{n}$, as transformadas de Fourier de $\nu_{n}$ e da função de correlação (5.8). Os números aleatórios com função de correlação (5.8) serão dados pela transformada inversa de Fourier de

$$
\tilde{\xi_{n}}=\left\{\tilde{G}_{n}\right\}^{1 / 2} \tilde{\nu}_{n}
$$

O ruído correlacionado $\xi_{n}$ com média $v$ e variância $\sigma^{2}$ pode ser obtido multiplicando estes números aleatórios por $\sigma$ e somando uma constante $v$.

Nas próximas seções apresentaremos os resultados das simulações. Iniciaremos com a análise da transição dos regimes $v=0$, que será seguida pelo estudo dos regimes 


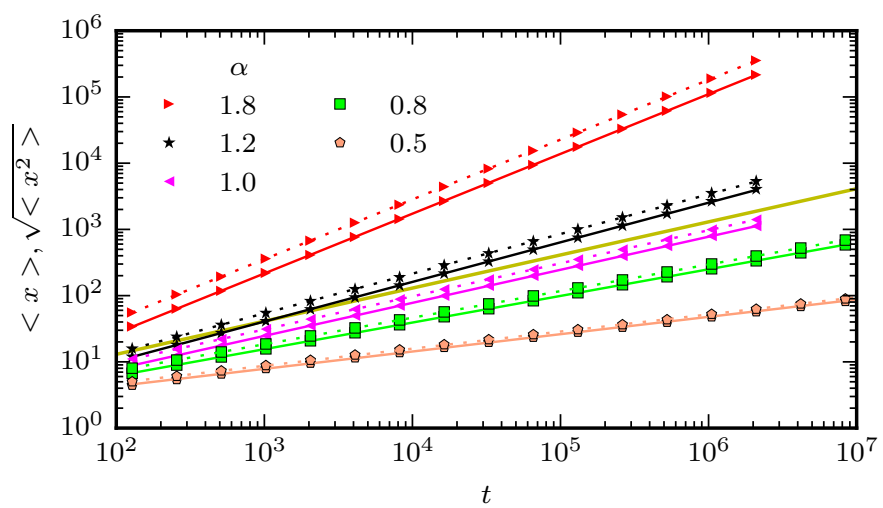

Figura 5.1: Média e raiz quadra do segundo momento da posição $x$ do movimento Browniano fracionário refletido na transição de regimes $(v=0)$ para diversos valores do expoente de difusão anômala $\alpha$. As linhas tracejadas e sólidas indicam ajustes em lei de potência. A linha sólida amarela indica o comportamento de difusão normal $\langle x\rangle \sim t$ com prefator arbitrário. A incerteza é da ordem de $1 \%$.

localizado e balístico. As simulações na transição de regime têm um tempo máximo de $2^{26} \approx 6.7^{7}$ e usam um número máximo de $10^{8}$ caminhantes. Além disso, usaremos a implementação (5.12) para a parede refletora. A menos que seja explicitamente mencionado, todas as simulações começarão em $x=0$ e o ruído terá largura $\sigma=1$.

\subsubsection{Simulações na transição de regimes $v=0$}

Começaremos o estudo da transição de regimes do movimento Browniano fracionário refletido analisando a média $\left\langle x_{n}\right\rangle$ e a raiz quadrada do segundo momento $\sqrt{\left\langle x_{n}^{2}\right\rangle}$ da posição $x$. A figura 5.1 mostra os gráficos de $\left\langle x_{n}\right\rangle$ e $\sqrt{\left\langle x_{n}^{2}\right\rangle}$ em função do tempo para diversos valores do expoente de difusão anômala $\alpha$. Os dados são compatíveis com leis de potência no tempo, e os ajustes em lei de potência de $\sqrt{\left\langle x_{n}^{2}\right\rangle}$ retornam os expoentes $0.903(5), 0.598(3), 0.499(3), 0.402(2)$ e $0.255(10)$, para os valores de $\alpha=1.8,1.2,1.0$, 0.8 e 0.5 , respectivamente. Estes valores mostram que a parede refletora não altera o comportamento de difusão anômala $\sqrt{\left\langle x^{2}\right\rangle} \sim t^{\alpha / 2}$ observado no caso não confinado. Além disso, como a parede refletora restringe o caminhante a valores positivos de $x$, a média $\left\langle x_{n}\right\rangle$ também se escala com $t^{\alpha / 2}$.

Apesar do resultado esperado para $\left\langle x_{n}\right\rangle$ e $\sqrt{\left\langle x_{n}^{2}\right\rangle}$, o mesmo não ocorre com a densidade de probabilidade da posição $P(x, t)$. A figura 5.2 mostra a densidade de probabilidade, em escala log-linear, para $\alpha=1.8$ (superdifusão ou correlações positivas) em vários instantes de tempo. Podemos ver que $P(x, t)$ apresenta uma singularidade próximo à $x=0$ (próximo à parede refletora), em contraste com a densidade de pro- 


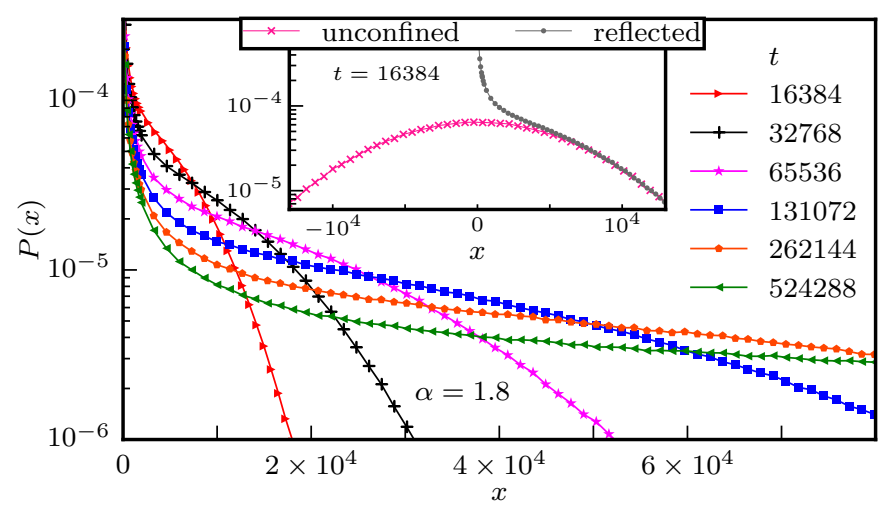

Figura 5.2: Densidade de probabilidade $P(x, t)$ da posição do caminhante $x$ no tempo $t$ em escala log-linear para o expoente de difusão anômala $\alpha=1.8$ (que corresponde a superdifusão ou correlações positivas). Inset: comparação entre as densidades de probabilidade do movimento Browniano fracionário não confinado e refletido no tempo $t=16384$. A incerteza é menor que o tamanho dos pontos.

babilidade gaussiana (5.11) para o caso não confinado. No inset podemos ver como a densidade de probabilidade no caso confinado difere da do caso não confinado.

Apesar do comportamento singular da densidade de probabilidade no caso refletido, esta densidade de probabilidade em diferentes instantes de tempo poder ser colapsada se plotada em função de $x /\left\langle x^{2}\right\rangle^{1 / 2}=x / \sigma t^{\alpha / 2}$. A figura 5.3 mostra o gráfico de $\left\langle x^{2}\right\rangle^{1 / 2} P$ vs. $x /\left\langle x^{2}\right\rangle^{1 / 2}$, para valores de $\alpha=1.8$ (superdifusão) e 0.8 (subdifusão) em escala log-log. Os desvios para $x /\left\langle x^{2}\right\rangle^{1 / 2}$ pequeno ocorrem devido à natureza discreta da implementação do movimento Browniano fracionário e tendem a ser cada vez menores com o aumento do tempo, quando a largura da densidade de probabilidade $\sqrt{\left\langle x^{2}\right\rangle} \sim t^{\alpha / 2}$ também aumenta. Este colapso da densidade de probabilidade sugere que a mesma pode ser escrita na forma

$$
P(x, t)=\frac{1}{\sigma t^{\alpha / 2}} Y\left(\frac{x}{\sigma t^{\alpha / 2}}\right) .
$$

O inset mostra que a cauda da densidade de probabilidade é gaussiana.

O colapso dos dados para valores de $x$ pequenos nos mostra que o comportamento singular observado na figura 5.3 não é um efeito de tempo finito e faz parte da função mestra $Y$. Além disso, a figura 5.3 mostra que a singularidade da densidade de probabilidade é compatível com a lei de potência

$$
P \sim x^{\kappa}
$$




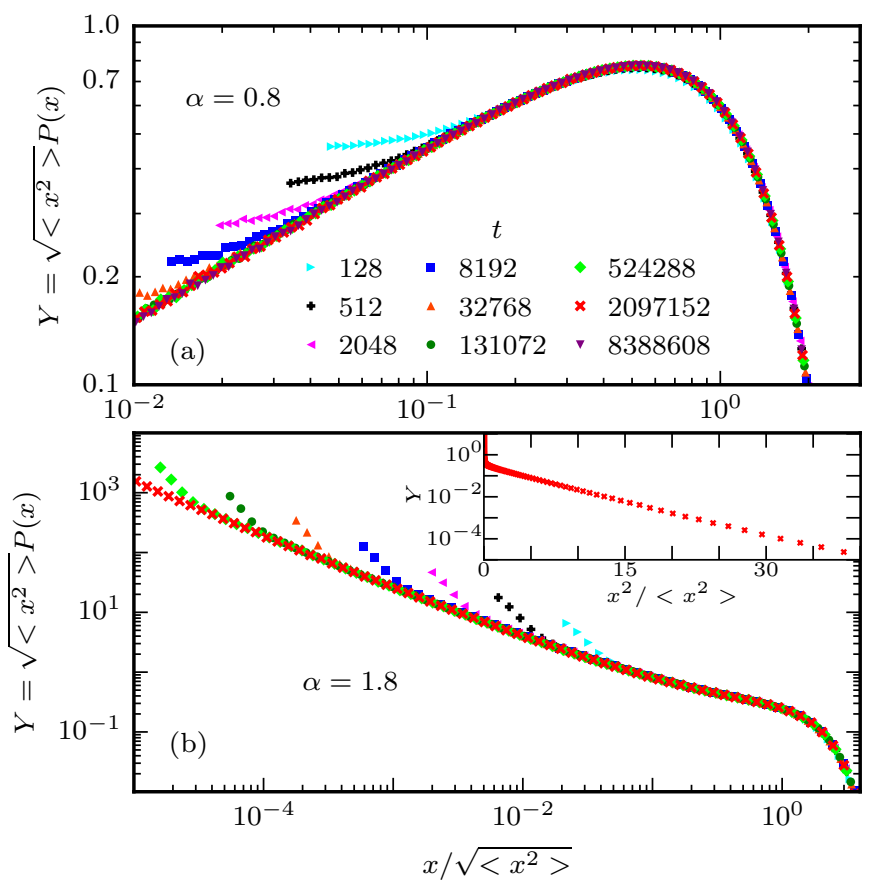

Figura 5.3: Gráfico de $\left\langle x^{2}\right\rangle^{1 / 2} P$ vs. $x /\left\langle x^{2}\right\rangle^{1 / 2}$ em escala log-log em diversos instantes de tempo. Os painéis (a) e (b) mostram simulações para $\alpha=0.8$ (subdifusão) e 1.8 (superdifusão), respectivamente. Inset: $\left\langle x^{2}\right\rangle^{1 / 2} P$ vs. $x^{2} /\left\langle x^{2}\right\rangle$ em escala log-linear de forma que o comportamento de uma gaussiana se assemelhe a uma linha reta. As incertezas são da ordem do tamanho dos símbolos.

A figura 5.4 mostra a densidade de probabilidade na forma escalada $\left(\left\langle x^{2}\right\rangle^{1 / 2} P\right.$ vs. $x /\left\langle x^{2}\right\rangle^{1 / 2}$ ) e em escala log-log para diversos valores de $\alpha$. Nesse gráfico podemos ver que a densidade de probabilidade diverge quando $\alpha>1$ (superdifusão), e tende a zero se $\alpha<1$ (subdifusão), implicando $\kappa<0$ se $\alpha>1$ e $\kappa>0$ se $\alpha<1$. No caso descorrelacionado $\alpha=0$, vemos que a densidade de probabilidade tende a uma constante, em concordância com a solução da equação de difusão para o caso refletido (5.3) quando $v=0$.

Agora passaremos para a análise do expoente da singularidade $\kappa$. Efetuando ajustes em lei de potência na densidade de probabilidade para valores pequenos de $x$, obtemos os valores de $\kappa$ plotados na figura 5.5. Os valores de $\kappa$ para $\alpha \leq 0.7$, ou seja, valores $\alpha$ de no regime subdifusivo, apresentam forte dependência com o tempo e por isso foram extrapolados conforme o inset na figura 5.5. Esta dependência com o tempo pode ser entendida como uma correção devido a tempos finitos: note na figura 5.3 que no regime subdifusivo $(\alpha<1)$ a densidade de probabilidade é muito menos larga e 


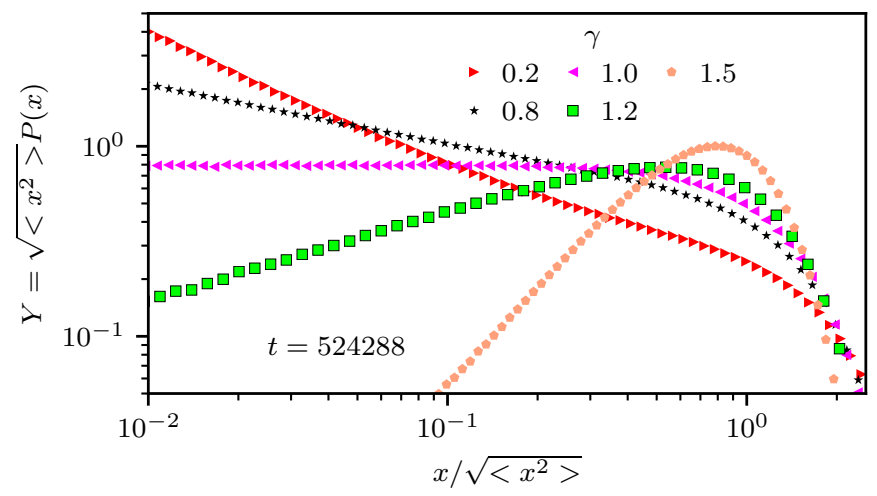

Figura 5.4: Densidade de probabilidade $P$ vs. $x$ em escala log-log para diversos valores do expoente de difusão anômala $\alpha$. As incertezas são menores que o tamanho do ponto.

portanto apresenta um intervalo em lei de potência muito menor se comparada com o caso superdifusivo $(\alpha>1)$.

A dependência do expoente $\kappa$ com $\alpha$ não é conhecida, no entanto vemos na figura 5.5 que os valores obtidos através dos ajustes são compatíveis com a forma

$$
\kappa=\frac{2}{\alpha}-2 \text {. }
$$

No limite do ruído perfeitamente anticorrelacionado, $\alpha \rightarrow 0, \kappa$ diverge. Por outro lado, se o ruído apresentar correlações positivas perfeitas, $\alpha \rightarrow 2, \kappa$ tende a -1 . Como $\kappa^{-1}$ não é normalizável, isto significa que a densidade de probabilidade tende a uma função delta.

O limite de correlações positivas perfeitas $(\alpha \rightarrow 2)$ corresponde ao movimento balístico das partículas, em que o sinal da velocidade é decidido a partir do ruído inicial $\xi_{1}$. Se $\xi_{1}<0$, as partículas ficam presas na parede refletora e a densidade de probabilidade é a função delta, em concordância com a análise do parágrafo anterior. Se $\xi_{1}>0$, os caminhantes propagam-se com velocidade balística para longe da parede refletora.

A singularidade na densidade de probabilidade aparece devido à interação entre a parede refletora e as correlações de longo alcance. Como podemos relacionar o acúmulo (divergência de $P$ ) e a ausência $(P \rightarrow 0)$ de caminhantes com as correlações positivas e negativas? Conforme a figura 5.6, podemos ver que para subdifusão (ou correlações negativas $\alpha<1$ ) um caminhante que atinge a parede refletora tende a se afastar da mesma em tempos subsequentes. Isto se deve às correlações negativas, pois estas fazem 


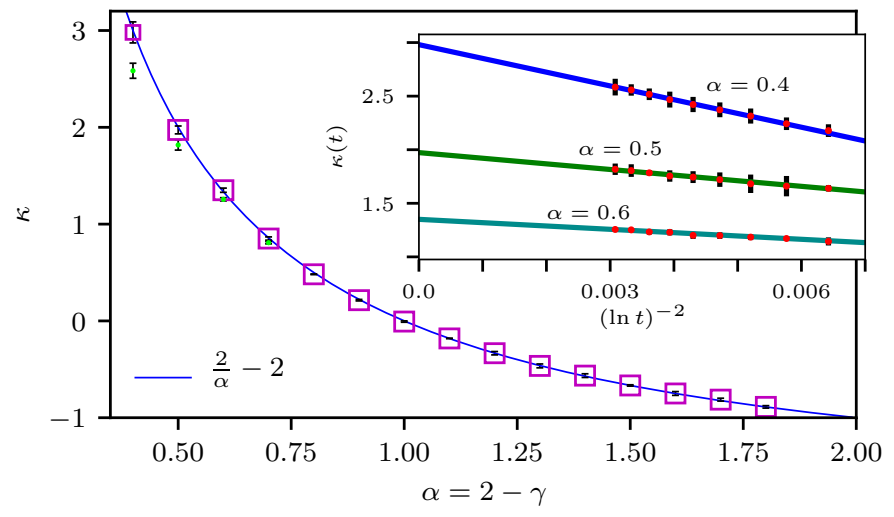

Figura 5.5: Expoente da singularidade (5.16) $\kappa$ em função do expoente de difusão anômala $\alpha$. Os quadrados representam os valores do expoente $\kappa$ e a linha sólida a função $2 / \alpha-2$. Os valores do expoente $\kappa$ para $\alpha=0.4,0.5,0.6$ e 0.7 apresentam grande variação com o tempo e foram extrapolados em função de $(\ln t)^{-2}$ conforme o inset. Os círculos verdes para estes mesmos valores de $\alpha$ representam o valor de $\kappa$ no maior tempo de simulação (sem extrapolação).

com que seja mais provável que o caminhante se desloque em direções opostas em tempos subsequentes. $\mathrm{O}$ oposto ocorre quando as correlações são positivas $(\alpha>1)$. Um caminhante deslocando-se em uma determinada direção tende a continuar andando nesta mesma direção, forçando os caminhantes com velocidade negativa a ficarem presos perto da parede refletora.

Para completar o argumento do parágrafo anterior, mostramos $\xi_{n}$ nos painéis superiores da figura 5.7 e o sinal de $\xi_{n}\left(\operatorname{sgn}\left(\xi_{n}\right)\right)$ no painéis inferiores. No caso de superdifusão $\alpha>1$, vemos que as correlações positivas aumentam a chance de encontrar valores consecutivos de $\xi_{n}$ com o mesmo sinal, ou seja, as correlações positivas aumentam a probabilidade de encontrar intervalos de tempo raros. O exato oposto ocorre quando as correlações são negativas $\alpha<1$.

\subsubsection{Teoria dos regimes localizado $(v<0)$ e balístico $(v>0)$}

Elaboraremos a teoria do regime localizado com base nos resultados descobertos na transição entre os regimes localizado e a balístico. Começaremos descrevendo o comportamento do comprimento de correlação temporal $\xi_{t}$ e da posição estacionária $x_{\mathrm{st}}$, depois analisaremos o comportamento da densidade de probabilidade. Por fim, estudaremos o comportamento do tempo $\tau$ que o caminhante leva para alcançar uma posição $x$ pela primeira vez.

No regime localizado, o caminhante está sujeito a uma velocidade de drift $v<0 \mathrm{em}$ 


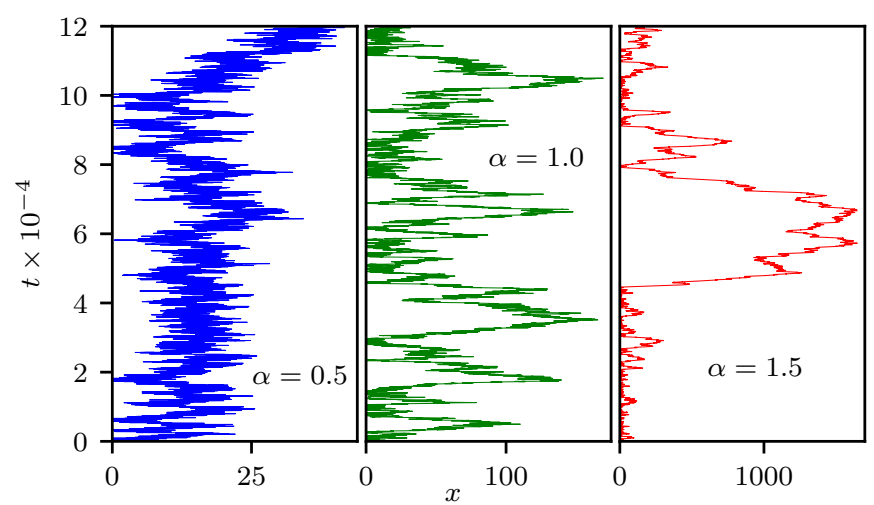

Figura 5.6: Trajetórias do movimento Browniano refletido nos casos de subdifusão $(\alpha=0.5)$, difusão normal $(\alpha=1.0)$ e superdifusão $(\alpha=1.5)$.
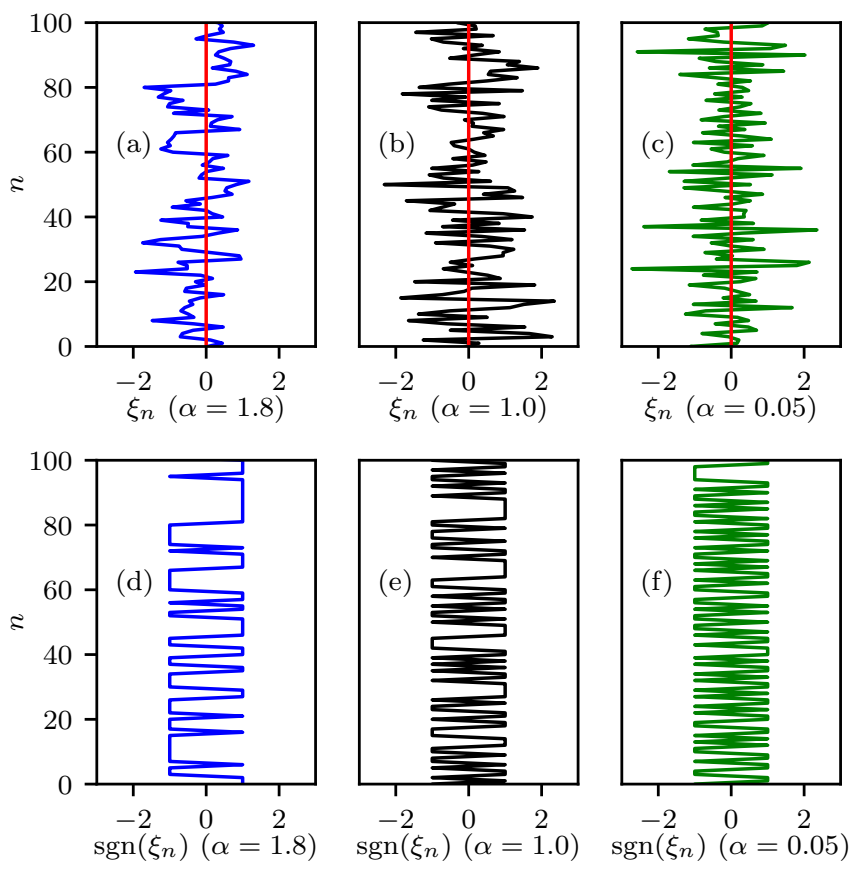

Figura 5.7: (a, b, c) Ruído fracionário gaussiano $\xi_{n}$ nos casos de superdifusão $(\alpha>1)$, difusão normal $(\alpha=1)$ e subdifusão $(\alpha<1)$. (d, e, f) Sinal do ruído fracionário gaussiano. 
direção à parede refletora, que dominará sobre o ruído no limite de tempos longos. O tempo de correlação $\xi_{t}$ será o tempo no qual o movimento balístico $v t$ começa a dominar sobre comportamento de difusão anômala $t^{\alpha / 2}$ observado na transição de regime. Este argumento nos leva a

$$
\xi_{t} \sim|v|^{-\nu_{\|}}
$$

com

$$
\nu_{\|}=\frac{2}{2-\alpha} .
$$

Após o tempo $\xi_{t}$, a velocidade de drift (negativa) domina sobre o ruído e os caminhantes, impedidos pela parede refletora de acessar o eixo negativo de $x$, ficam presos perto da mesma. Devido ao confinamento dos caminhantes, a posição média atinge um valor estacionário $x_{\mathrm{st}}$ finito que pode ser estimado como a posição no tempo $\xi_{t}$

$$
x_{\mathrm{st}} \sim|v|^{\beta_{x}},
$$

no qual

$$
\beta_{x}=\frac{\alpha}{2-\alpha} .
$$

$\mathrm{Na}$ terminologia de fenômenos críticos, o comportamento descrito por (5.18) e (5.20) deixa evidente que $|v|$ assume o papel de distância ao ponto crítico, como no caso de difusão normal com a parede refletora $5.1^{1}$.

O confinamento dos caminhantes perto da parede refletora faz com que a densidade de probabilidade também atinja o estado estacionário $P_{\mathrm{st}}$. Analogamente ao escalamento da densidade de probabilidade na transição de regime (5.15), o ansatz para a densidade de probabilidade estacionária no regime localizado será

$$
P_{\mathrm{st}}=|v|^{\beta_{x}} Y_{\mathrm{st}}\left(x|v|^{\beta_{x}}\right) .
$$

Perceba que a combinação entre $x$ e $|v|$ encontrada no argumento de $Y_{\text {st }}$ segue de (5.20).

Se supormos que a mesma singularidade em lei de potência (5.16) está presente no regime localizado, isto é, $Y_{\text {st }}(z) \sim z^{\kappa}$ para $z$ pequeno, podemos concluir que

$$
P_{\mathrm{st}} \sim|v| x^{\kappa}
$$

quando $x \rightarrow 0^{2}$.

\footnotetext{
${ }^{1}$ No entanto, note que $\left\langle x_{n}\right\rangle$ não é um parâmetro de ordem, pois não assume um valor nulo em nenhuma dos regimes

${ }^{2}$ Embora não tenhamos prova de que a singularidade presente na transição de regime existe no
} 
Após descrevermos o comportamento da densidade de probabilidade perto da parede refletora (5.23), nos resta entender como a cauda da densidade de probabilidade decai para valores grandes de $x$. Dado que estamos no regime localizado onde a velocidade de drift força os caminhantes a ficarem perto da parede refletora, um caminhante só pode ser encontrado longe desta parede se houver um intervalo de tempo raro $T_{\mathrm{RR}}$ onde as flutuações devido ao ruído $\xi_{i}$ sobrepõem a velocidade de drift. A seguir faremos a análise da cauda da densidade de probabilidade a partir desta idéia.

Primeiramente, definiremos o ruído efetivo $\xi_{\mathrm{RR}}$ como a média dos ruídos no intervalo de tempo raro $T_{\mathrm{RR}}$,

$$
\xi_{\mathrm{RR}}=\frac{1}{T_{\mathrm{RR}}} \sum_{i \in \mathrm{RR}} \xi_{i}
$$

A probabilidade de encontrar essa sequencia de ruídos $\xi_{i}, \ldots, \xi_{i+T_{\mathrm{RR}}}$ é dada pela gaussiana correlacionada

$$
P_{\xi} \sim \exp \left\{-\frac{1}{2} \sum_{i, j \in \mathrm{RR}}\left(\xi_{i}-v\right) A_{i j}\left(\xi_{j}-v\right)\right\},
$$

onde $A_{i j}=G^{-1}(i-j)$.

Seguindo a referência [39], a probabilidade de encontrar um intervalo de ruído efetivo $\xi_{\mathrm{RR}}$ pode ser deduzida introduzindo $\xi_{\mathrm{RR}}$ com uma nova variável em (5.25) e integrando todas as outras. A densidade de probabilidade resultante será

$$
P_{\mathrm{RR}} \sim \exp \left\{-\frac{T_{\mathrm{RR}}^{2-\alpha}}{2 b^{2}}\left(\xi_{\mathrm{RR}}-v\right)^{2}\right\} .
$$

Da equação (5.26) vemos que correlações positivas $(\alpha>1)$ aumentam a probabilidade de encontrar essas regiões raras.

Como estamos supondo que o ruído efetivo domina sobre a velocidade de drift, podemos aproximar $x \approx T_{\mathrm{RR}} \xi_{\mathrm{RR}}$. Substituindo esta aproximação na equação (5.26) e usando o método do ponto de sela para integrar todos os $\xi_{\mathrm{RR}}>0$, obtemos

$$
P_{\mathrm{st}}(x, v) \sim \exp \left\{-C|v|^{\alpha} x^{2-\alpha}\right\}
$$

que deve descrever o comportamento da cauda da densidade de probabilidade para $x$ suficientemente grande no estado estacionário. Além disso, a densidade de probabilidade (5.27) concorda com a forma de escala (5.15) se $Y_{\text {st }}(z) \sim \exp \left\{-z^{2-\alpha}\right\}$. No caso do ruído 
descorrelacionado $\alpha=1$, a equação (5.27) concorda com a exponencial simples para o caso de difusão normal (veja a seção 5.1).

O tempo típico $\tau$ que o caminhante leva para chegar na posição $x$ é proporcional ao inverso da densidade de probabilidade, logo

$$
\tau \sim \exp \left\{C|v|^{\alpha} x^{2-\alpha}\right\}
$$

No regime balístico $v>0$, o caminhante tende a se deslocar balisticamente para longe da parede refletora no limite de tempos longos. Sendo assim, esperamos que os efeitos da parede refletora desapareçam no limite de tempos longos e a densidade de probabilidade deve ser a mesma gaussiana observada no movimento Browniano fracionário não confinado (5.11).

\subsubsection{Simulações nos regimes localizado $(v<0)$ e balístico $(v>0)$}

Nesta subseção mostraremos os resultados das simulações no regime localizado para verificar a validade da teoria.

Primeiramente verificaremos a implementação da parede refletora. Diferente da transição de regime e do regime balístico, no regime localizado as partículas ficam confinadas perto da parede refletora fazendo com que a largura da densidade de probabilidade permaneça finita. Isso significa que diferentes implementações da parede refletora podem levar a diferentes correções devido à natureza discreta das simulações, quando $x$ e $\sigma$ são da mesma ordem.

A figura 5.8 mostra a densidade de probabilidade estacionária obtida através das simulações no caso de ruído descorrelacionado $(\alpha=1)$ para as duas implementações da parede refletora (5.12) e (5.13). A linha sólida mostra a solução (5.3) da equação de difusão com a parede refletora no limite $t \rightarrow \infty$. Neste gráfico, podemos ver que a densidade de probabilidade resultante das simulações usando a implementação da parede refletora (5.12) apresenta desvios significativos da solução analítica quando $|v|$ é grande e a densidade de probabilidade não se expande. No entanto, a diferença entre as simulações com a equação (5.12) e a solução analítica diminuem quando nos aproximamos do ponto crítico $v=0$, ou quando a densidade de probabilidade torna-se mais larga. De fato, a diferença entre as simulações e a solução analítica está dentro da incerteza para $v=-0.02$. Por outro lado, vemos que as simulações usando a implementação da parede refletora (5.13) apresentam melhor concordância com a solução analítica, logo usaremos a implementação (5.13) para as simulações no regime localizado. 


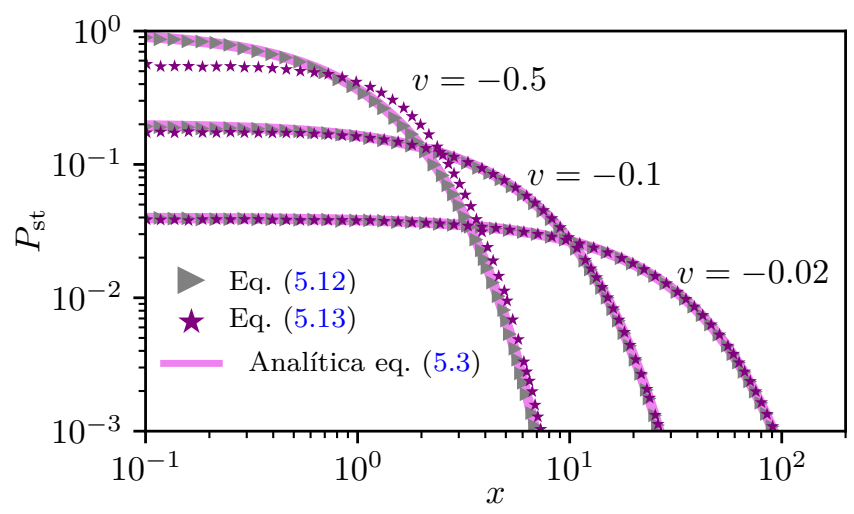

Figura 5.8: Densidade de probabilidade no regime localizado para $\alpha=1$ (ruído descorrelacionado) e diferentes implementações da parede refletora.

Agora que definimos a implementação da parede refletora, verificaremos o comportamento qualitativo de $\langle x\rangle$ nos regimes localizado e balístico. Conforme a figura 5.9(a), vemos que no regime balístico $\langle x\rangle$ tende a $v t$ no limite de tempos longos e que $\langle x\rangle$ tende a um valor finito no regime localizado, conforme esperado.

Verificado o comportamento qualitativo dos diferentes regimes, passamos para a análise quantitativa dos dados. A posição estacionária $x_{\text {st }}$ pode ser estimada ajustando uma constante no comportamento assintótico de $\langle x\rangle$, conforme a figura 5.9(a). Os valores de $x_{\text {st }}$ obtidos para diversos valores do expoente de difusão anômala $\alpha$ estão plotados na figura 5.10(a). Nesses dados, vemos que $x_{\text {st }}$ segue leis de potência em determinados intervalos de $|v|$ para todos os valores de $\alpha$, em concordância com $x_{\mathrm{st}} \sim|v|^{\beta_{x}}$ (5.20). Os desvios do comportamento em lei de potência para $|v|$ pequeno e grande se devem ao longo crossover para o comportamento estacionário próximo ao ponto crítico, e às discrepâncias devido à natureza discreta das simulações quando a densidade de probabilidade é estreita. Os valores estimados do expoente $\beta_{x}$ estão plotados na figura $5.9(\mathrm{c})$, no qual vemos uma boa concordância com a teoria.

O comprimento de correlação temporal pode ser estimado como o tempo $t_{x}$ em que as curvas no regime balístico intersectam a metade da curva crítica $x_{\text {crit }} / 2$, conforme ilustrado na figura 5.9(b). Os valores de $t_{x}$ estão plotados na figura 5.10(b), onde podemos ver que $t_{x}$ segue leis de potência para vários valores de $\alpha$. Os valores dos expoentes ajustados estão na figura 5.10(c), onde vemos boa concordância com o valor de $\nu_{\|}$esperado.

Após analisar o comportamento de $x_{\mathrm{st}}$ e $\xi_{t}$, voltamos a atenção para a densidade de probabilidade. Iniciaremos discutindo como a densidade de probabilidade se apro- 

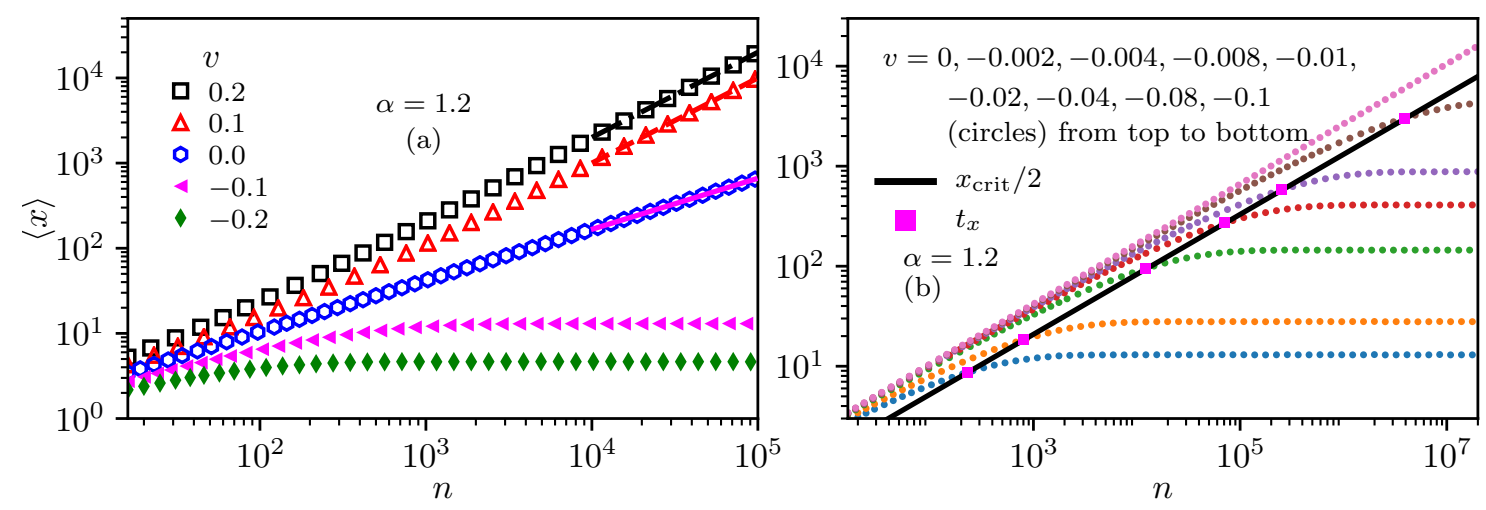

Figura 5.9: (a) Posição média $\langle x\rangle$ em função do tempo no regime balístico $(v>0)$, na transição de regime $(v=0)$ e no regime localizado $(v<0)$. No regime balístico $\langle x\rangle$ tende assintoticamente ao movimento balístico $v t$, representado pelas linhas tracejadas. $\mathrm{Na}$ transição de regime observamos o comportamento de difusão anômala $\langle x\rangle \sim t^{\alpha / 2}$. No regime localizado $\langle x\rangle$ atinge um valor finito. (b) Simulações mais detalhadas no regime localizado. As incertezas são menores que o tamanho dos símbolos.
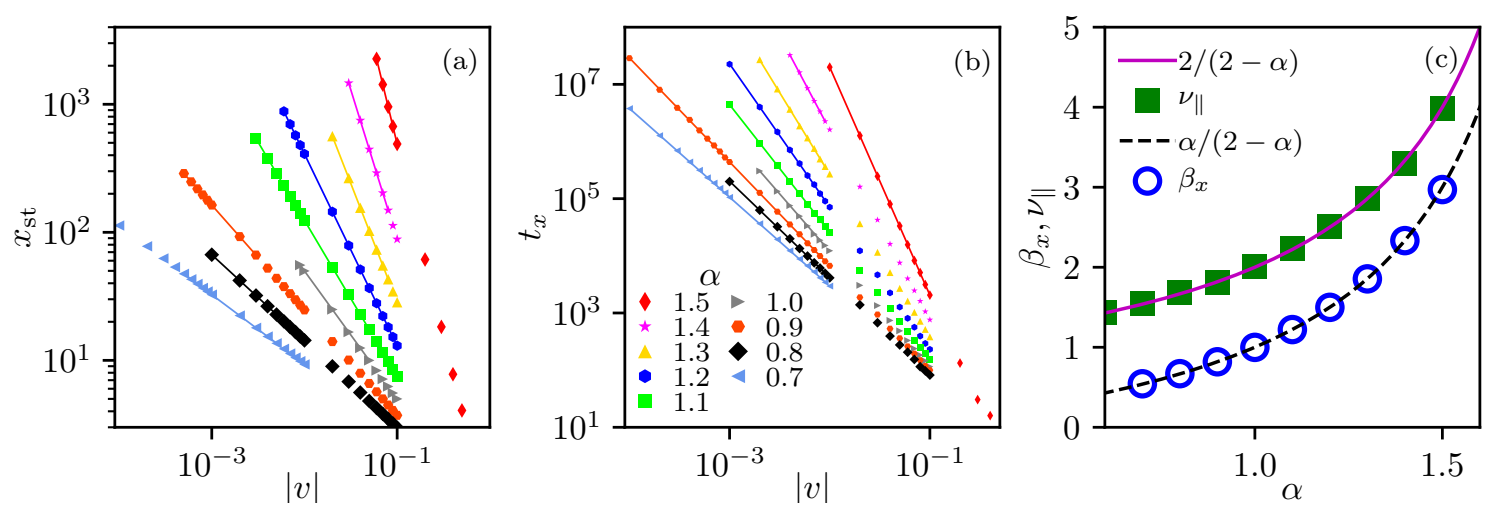

Figura 5.10: (a) Posição estacionária $x_{\text {st }}$ em função da distância ao ponto crítico $|v|$. (b) Tempo de correlação $t_{x}$ vs. $|v|$. (c) Expoentes extraídos dos dados dos painéis (a) e (b). As linhas sólidas indicam os expoentes preditos $\nu_{\|}=2 /(2-\alpha)$ e $\beta_{x}=\alpha /(2-\alpha)$ de acordo com as equações (5.18) e (5.20). As incertezas são da ordem do tamanho dos pontos. 

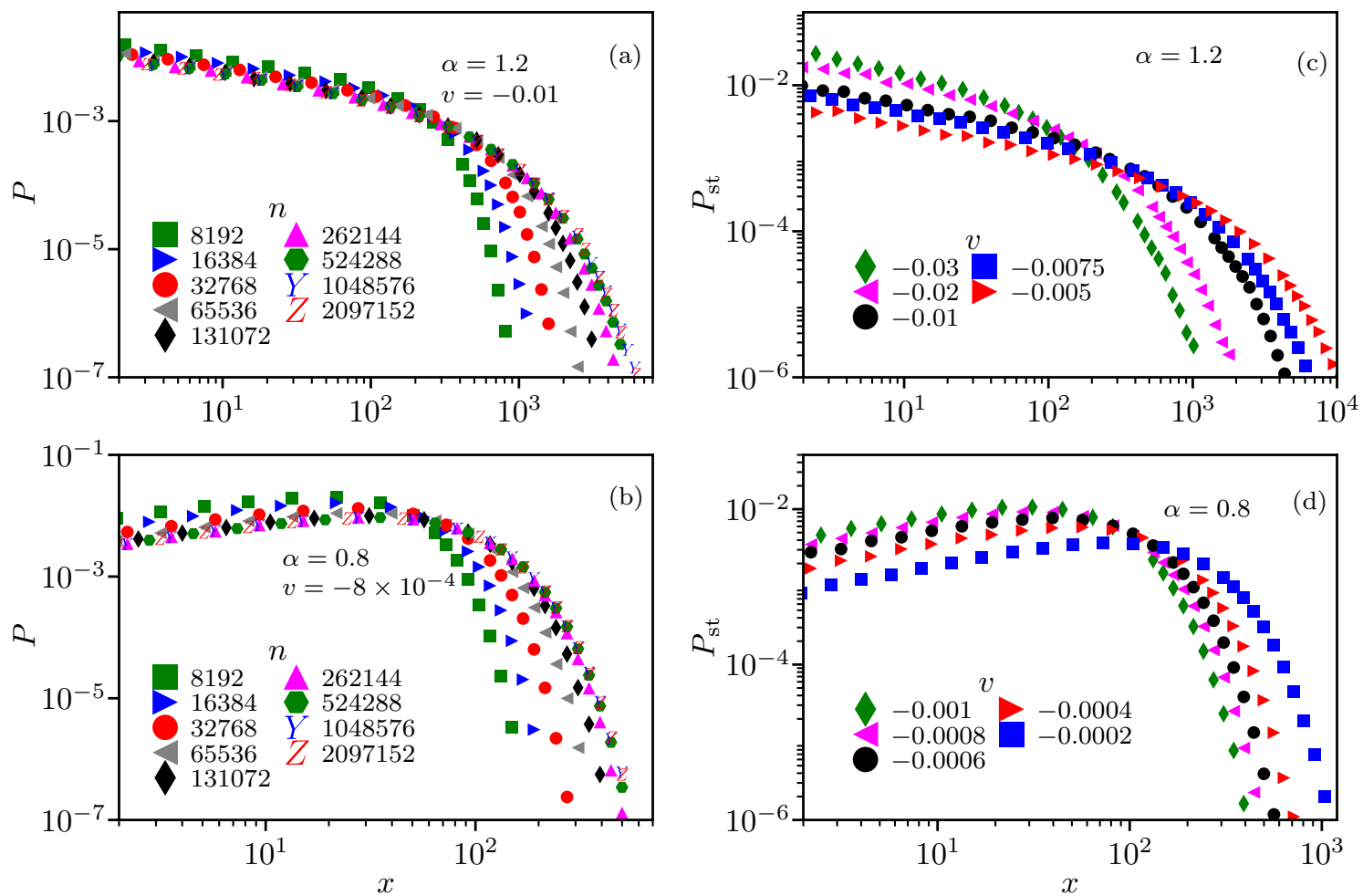

Figura 5.11: (a,b) Densidade de probabilidade em função do tempo no regime localizado, para os regimes super e sub difusivos e $v$ fixo. Em ambos os casos, as densidades de probabilidade para tempos superiores a 524288 concordam dentro das incertezas, portanto podem ser consideradas estacionárias. (c, d) Densidade de probabilidade estacionária $P_{\text {st }}$ para diversos valores de $v$. As incertezas são da ordem do tamanho dos símbolos.

xima do estado estacionário $P_{\mathrm{st}}$. As figuras 5.11(a) e 5.11(b) mostram a densidade de probabilidade nos casos super e sub difusivos para um valor fixo de $v$ em vários instantes de tempo $n$. Em ambos os casos, as densidades de probabilidade podem ser consideradas estacionárias em tempos superiores a 524288. No entanto, perceba que precisamos de uma velocidade de drift de maior magnitude no caso superdifusivo para superar os efeitos das correlações positivas em tempos similares. As figuras 5.11(c) e 5.11(d) mostram as estimativas da densidade de probabilidade estacionárias $P_{\mathrm{st}}$ para vários valores de $v$. Nesses gráficos, em escala log-log, o comportamento visualmente linear da densidade de probabilidade quando $x$ é pequeno é uma evidência de que há uma singularidade na forma de uma lei de potência no regime localizado.

Após avaliar a densidade de probabilidade no estado estacionário, verificaremos se 
$P_{\text {st }}$ segue a função de escala (5.22). As figuras 5.12(a) e 5.12(b) mostram os gráficos de $|v|^{-\beta_{x}} P_{\text {st }}$ vs. $x|v|^{\beta_{x}}$, onde verificamos um colapso quase perfeito dos dados, em concordância com (5.22). Além disso, os gráficos de $|v|^{-1} P_{\text {st }}$ vs. $x$, nos painéis (c) e (d), mostram que a densidade de probabilidade estacionária difere apenas de uma constante $|v|$ para $x$ pequeno, em concordância com a equação (5.23), que é uma consequência da existência da mesma singularidade em lei de potência (5.16) verificada na transição de regime. De fato, o comportamento visualmente linear no gráfico log-log da densidade de probabilidade para valores pequenos de $x$ no regime localizado é paralelo ao observado na transição de fase $(v=0)$, sugerindo que o expoente da singularidade é o mesmo. Ajustes de leis de potência $x^{\kappa}$ (linhas cinza) no comportamento de $x$ pequeno no regime localizado retornam os expoentes $\kappa=-0.33(2)$ e $0.496(9)$ para $\alpha=1.2$ e 0.8 , em concordância com o valor conjecturado $\kappa=2 / \alpha-2$.

Agora que verificamos o comportamento singular de $P_{\text {st }}$ para valores pequenos de $x$, realizaremos simulações a fim de averiguar se comportamento de $P_{\text {st }}$ para $x$ grande segue a exponencial esticada (5.27). A figura 5.11 mostra $P_{\text {st }}$ vs. $x^{2-\alpha}$, para $\alpha=1.2 \mathrm{e}$ 0.8 , de forma que o comportamento predito por (5.27) seja visualmente linear. Como o comportamento de $P_{\mathrm{st}}$ se mostra compatível com a predição para vários valores de $|v|$ e diversas décadas em $P_{\mathrm{st}}$, efetuamos ajustes na forma $e^{-b x^{2-\alpha}}$ para verificar se $b \sim|v|^{\alpha}$. A figura 5.14(a) mostra a constante $b$ ajustada para diferentes valores de $\alpha$, onde podemos ver que $b$ varia conforme uma lei de potência em $|v|$. Em 5.14(b) vemos que os expoentes resultantes dos ajustes em lei de potência nos dados da figura 5.14(a) seguem muito bem o esperado.

Para terminar o estudo do regime localizado, faremos a análise do tempo $\tau$ que o caminhante leva para chegar na posição $x$ pela primeira vez. De forma semelhante à análise no caso do comportamento de $P_{\text {st }}$ para $x$ grande feito no parágrafo anterior, os painéis (a) e (b) da figura 5.15 mostram $\tau$ vs. $x^{2-\alpha}$ de forma que o comportamento descrito por (5.28) seja uma linha reta. Neste caso vemos que $\tau$ segue o comportamento descrito por (5.28) por aproximadamente três décadas quando $\alpha=1.2$, e duas quando $\alpha=0.8$. Sendo assim, realizamos ajustes de exponenciais $e^{b x^{2-\alpha}}$ para verificar se $b \sim|v|^{\alpha}$. O painel (c) mostra o fator $b$ vs. $|v|$ onde podemos ver uma boa compatibilidade com o comportamento em lei de potência se $|v|$ for pequeno. Os desvios quando $|v|$ é grande ocorrem pois não conseguimos alcançar o limite de $x$ suficientemente grande ${ }^{3}$.

\footnotetext{
${ }^{3}$ Note que, conforme $|v|$ aumenta (ou $v$ diminui), os caminhantes tendem a ficar mais confinados perto da parede refletora aumentado o tempo típico necessário para que a partícula leva para chegar na posição $x$.
} 

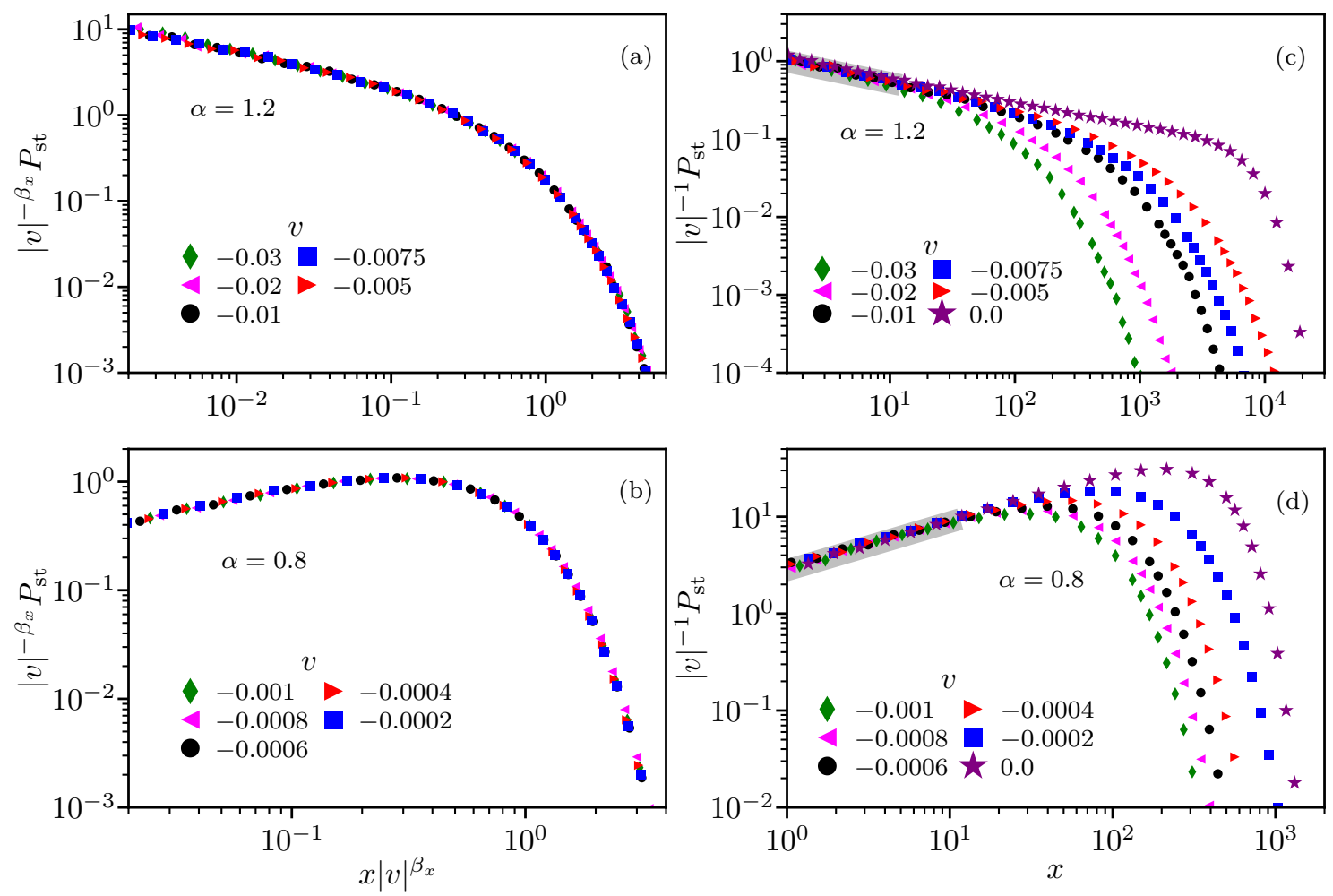

Figura 5.12: (a,b) Densidade de probabilidade em função do tempo no regime localizado, para os regimes super e sub difusivos e $v$ fixo. Em ambos os casos as densidades de probabilidade para tempos superiores a 524288 concordam dentro das incertezas, portanto podem ser consideradas estacionárias. (c, d) Densidade de probabilidade estacionária $P_{\text {st }}$ para diversos valores de $v$. As incertezas são da ordem do tamanho dos símbolos.
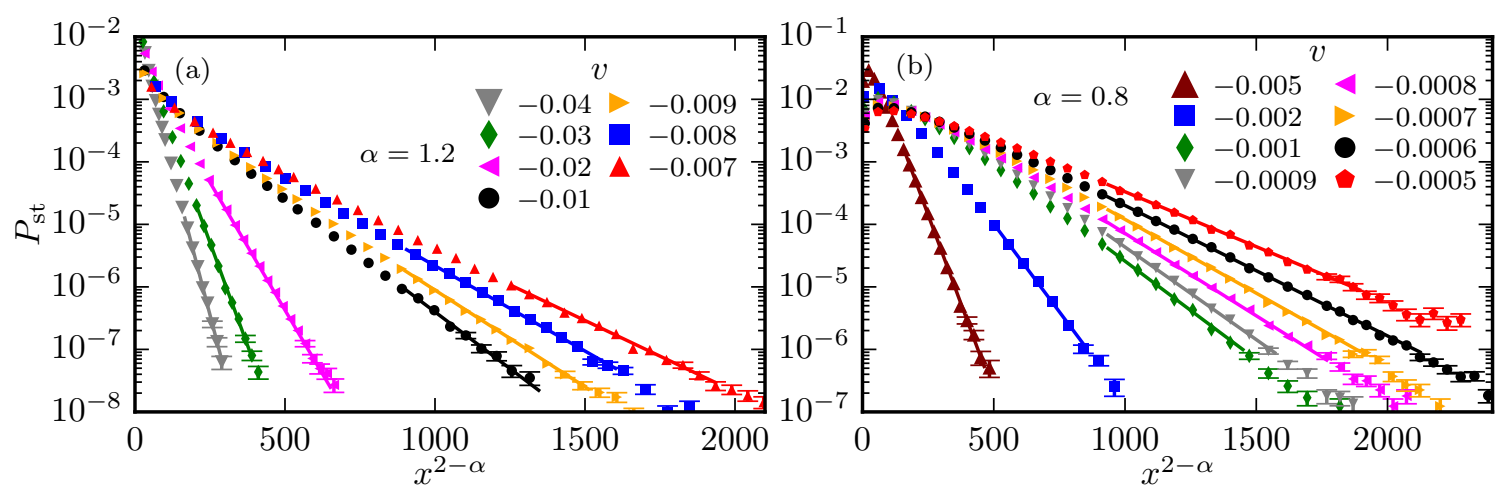

Figura 5.13: Densidade de probabilidade estacionária $P_{\mathrm{st}}$ vs. $x^{2-\alpha}$ para diversos valores de $|v|$ e $\alpha=1.2$ e 0.8 . As linhas sólidas são ajustes de exponenciais $e^{-b x^{2-\alpha}}$. As incertezas, se não mostradas, são menores que $10 \%$. 

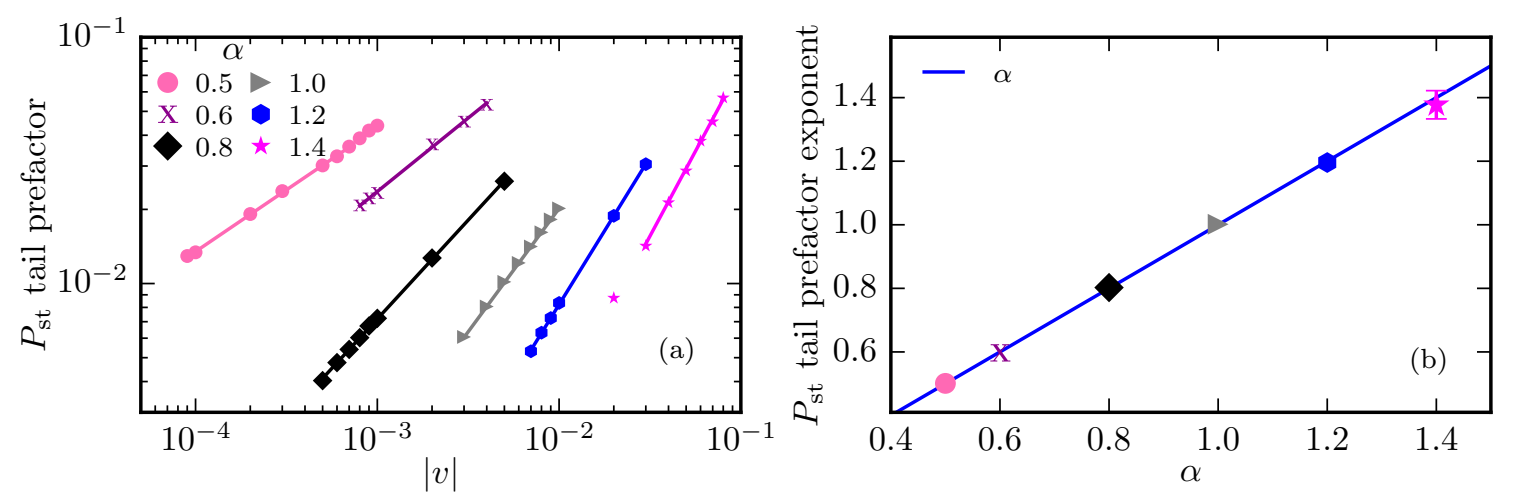

Figura 5.14: (a) Expoente $b$ do ajuste $P_{\mathrm{st}} \sim e^{-b x^{2-\alpha}}$ vs. $|v|$. As linhas sólidas são ajustes de leis de potência. (b) Expoentes extraídos a partir de ajustes de leis de potência dos dados do painel (a) em função de $|v|$. Os expoentes concordam com a predição $b \sim|v|^{\alpha}$. As incertezas são da ordem do tamanho dos símbolos.
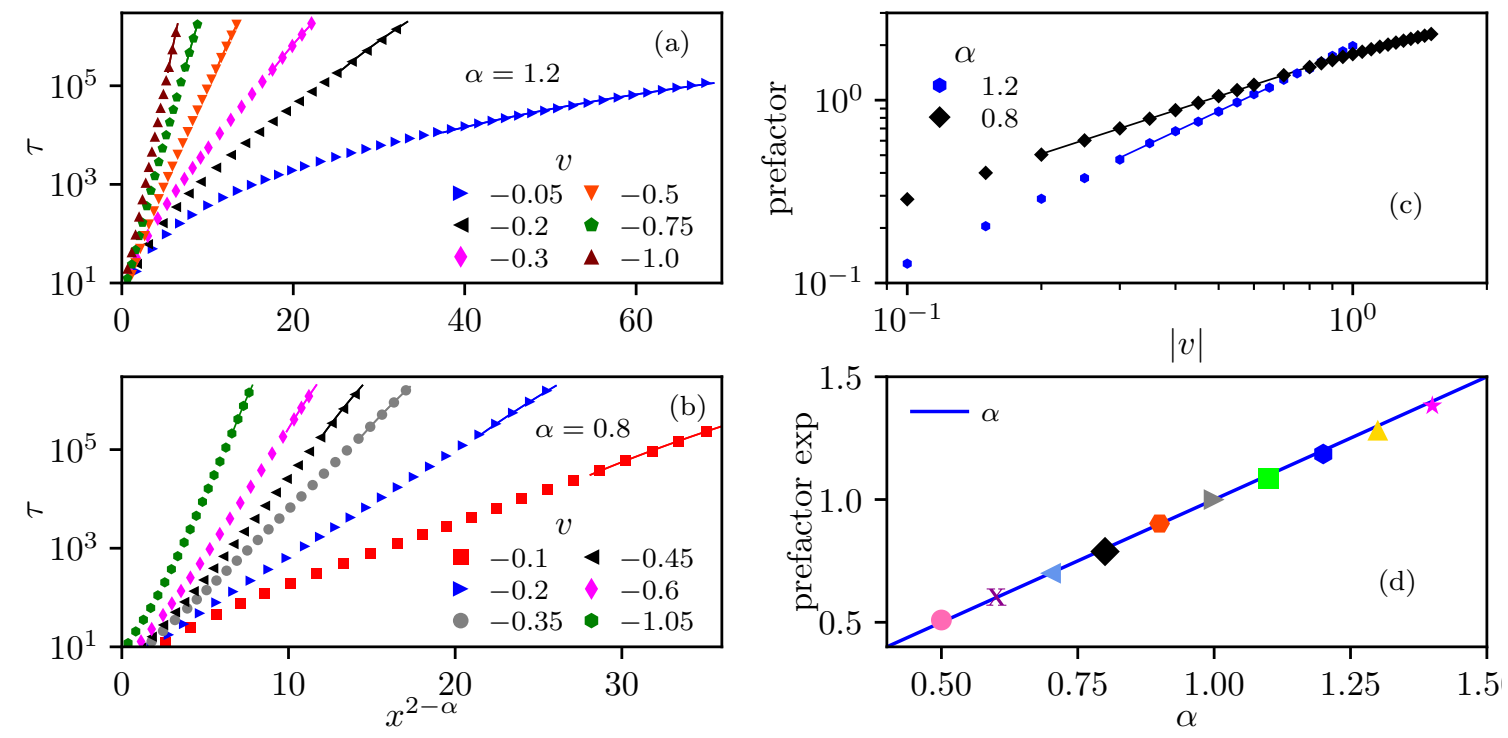

Figura 5.15: (a) Expoente $b$ do ajuste $P_{\mathrm{st}} \sim e^{-b x^{2-\alpha}}$ vs. $|v|$. As linhas sólidas são ajustes de leis de potência. (b) Expoentes extraídos a partir dos ajustes de leis de potência nos dados do painel (a) em função de $|v|$. Os expoentes concordam com a predição $b \sim|v|^{\alpha}$. As incertezas são da ordem do tamanho dos símbolos. 

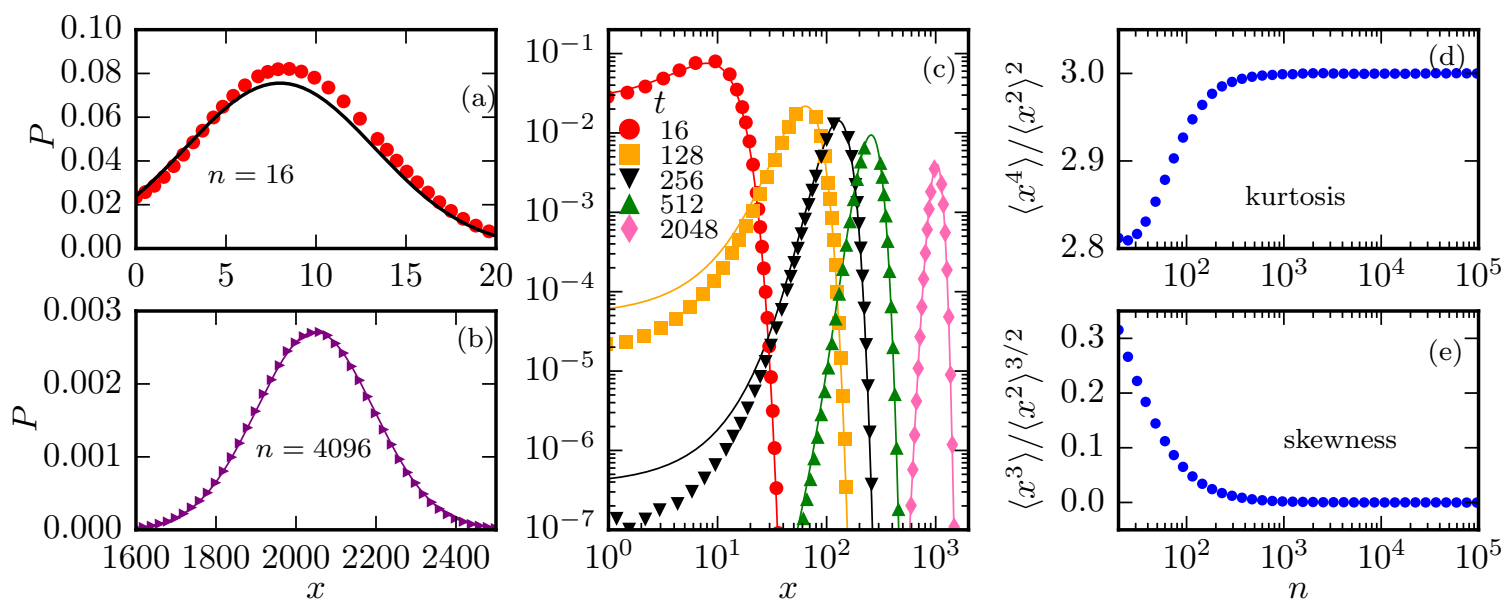

Figura 5.16: (a, b, c) Densidade de probabilidade em diversos instantes de tempo para $v=0.5$. As linhas sólidas representam a densidade de probilidade (5.11) para o caso não confinado. (d, e) Curtose e assimetria da densidade de probabilidade no caso refletido.

\subsubsection{Regime balístico $v>0$}

No regime balístico a posição média dos caminhantes se propaga com velocidade $v$, no limite de tempos longos, para longe da parede refletora. Sendo assim, esperamos que os efeitos devido à parede refletora desapareçam e que a densidade de probabilidade se comporte como no caso não confinado (5.11).

A figura 5.16 mostra a densidade de probabilidade em diversos instantes de tempo para $v=0.5$ e $\alpha=1.2$. Nesta figura as linhas sólidas representam a densidade de probabilidade gaussiana do movimento Browniano fracionário não confinado (5.11), e os pontos são as simulações para o caso refletido. No painel (a) vemos que quando $n=16$ a densidade de probabilidade no caso refletido difere significativamente do caso não confinado, no entanto, conforme o painel (c), a diferença entre estes dois casos diminui rapidamente com o tempo. No painel (b) podemos ver que a diferença entre os casos refletido e o não confinado é muito pequena. Os painéis (d) e (e) mostram que a curtose e a assimetria (skewness) da densidade de probabilidade no caso refletido tendem a o valor que se espera de uma gaussiana no limite de tempos longos. 



\section{Capítulo 6}

\section{Equação logística com desordem temporal correlacionada}

\subsection{Definição}

A equação logística [40]

$$
\frac{d \rho}{d t}=-\mu \rho+\lambda \rho(1-\rho)
$$

descreve a evolução da densidade de indivíduos $\rho$ com taxa de mortalidade e crescimento dadas por $\mu$ e $\lambda$. Se as taxas $\mu$ e $\lambda$ forem constantes, a solução de (6.1) é dada por

$$
\rho^{-1}=\rho_{0}^{-1} e^{(\mu-\lambda) t}+\lambda \frac{e^{(\mu-\lambda) t}-1}{\mu-\lambda}
$$

onde $\rho_{0}$ é a densidade de indivíduos no tempo $t=0$. Nesta solução podemos ver que a população atinge um valor estacionário (sobrevive) se $\lambda>\mu$ e decai exponencialmente se $\mu>\lambda$. Quando $\lambda=\mu$, observamos a transição de fase onde a densidade decai como uma lei de potência $\rho \sim t^{-1}$. A figura 6.1 ilustra a evolução de $\rho$ nos três casos descritos.

A equação logística, da maneira como foi introduzida, é desprovida de flutuações na densidade $\rho$. No entanto, flutuações são importantes na descrição da sobrevivência e extinção de populações biológicas, pois reduzem significativamente seu tempo de sobrevivência. Introduziremos flutuações temporais na equação logística (conforme [87]) permitindo que as taxas $\mu$ e $\lambda$ variem aleatoriamente no tempo, mas fixando-as em intervalos de tempo $\Delta t$, conforme a figura 6.1. As taxas em cada intervalo de tempo $\Delta t$ podem ser escritas nas formas $\mu_{n}=\Delta \mu_{n}+\mu$ e $\lambda_{n}=\Delta \lambda+\lambda$, sendo $n$ o número de intervalos de tamanho $\Delta t$ até o tempo $t$, e $\Delta \mu_{n}$ e $\Delta \lambda_{n}$ ruídos de média nula. 

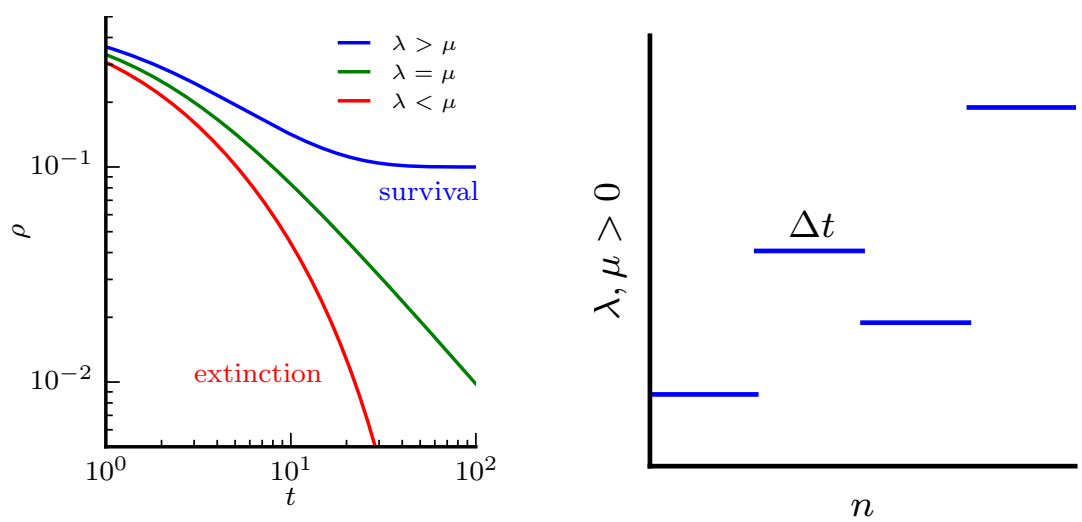

Figura 6.1: Esquerda: evolução logística da densidade de indivíduos $\rho$ dada pela equação (6.2). Se $\lambda>\mu$ a população sobrevive atingindo um valor estacionário de $\rho$. Se $\mu>\lambda$ a população decai exponencialmente. Entre a extinção e sobrevivência da população, em $\mu=\lambda$, temos a transição de fase onde $\rho \sim t^{-1}$. Direita: exemplo da desordem temporal utilizada. O eixo $y$ representa as taxas $\mu_{n}$ ou $\lambda_{n}$, e o eixo $x$ o tempo.

\subsection{Mapeamento no movimento Browniano fracionário re- fletido}

Como podemos resolver a equação logística com esse tipo de desordem temporal? Dado que as taxas são constantes em intervalos de tempo $\Delta t$, podemos usar a solução da equação logística (6.2) em cada um destes intervalos. Dessa forma, a evolução da densidade de indivíduos no início de cada intervalo de tamanho $\Delta t$ será dada por

$$
\rho_{n+1}^{-1}=\rho_{n}^{-1} a_{n}+c_{n},
$$

sendo $a_{n}=\exp \left\{\left(\mu_{n}-\lambda_{n}\right) \Delta t_{n}\right\}$ e $c_{n}=\left(a_{n}-1\right) \lambda_{n} /\left(\mu_{n}-\lambda_{n}\right)$.

Ignorando $c_{n}$, por um momento, e definindo $x_{n}=-\ln \rho_{n}$, podemos ver que a evolução de $\rho_{n}$ (6.3) pode ser mapeada num passeio aleatório $x_{x+1}=x_{n}+\xi_{n}$, com ruído

$$
\xi_{n}=\ln a_{n}=\left(\mu_{n}-\lambda_{n}\right) \Delta t .
$$

Logo, podemos definir a velocidade de drift como

$$
v=\frac{1}{\Delta t}\left\langle\xi_{n}\right\rangle=\mu-\lambda .
$$

Por simplicidade, neste trabalho usaremos $\Delta \lambda=0$ e apenas $\Delta \mu$ será responsável pela introdução de desordem. $c_{n}$ só é importante quando $\rho_{n} \approx 1$ e tem o efeito de impedir 
que $\rho_{n}$ cresça acima de 1 . Veremos que, devido ao mapeamento no passeio aleatório $x=\ln \rho, c_{n}$ pode aproximada por uma parede refletora na origem [87].

Devido ao mapeamento da equação logística com desordem temporal no passeio aleatório refletido, é conveniente usar um ruído $\xi_{n}$ com as mesmas propriedades do ruído gaussiano fracionário, isto é, $\xi_{n}$ será um ruído de densidade de probabilidade gaussiana com média $v$, variância $\sigma^{2}$, e função de correlação

$$
G(n)=\left\langle\xi_{m} \xi_{m+n}\right\rangle-\left\langle\xi_{m}\right\rangle\left\langle\xi_{m+n}\right\rangle=\frac{\sigma^{2}}{2}\left(|n+1|^{\alpha}-2|n|^{\alpha}+|n-1|^{\alpha}\right),
$$

com $\alpha$, o expoente de difusão anômala, variando entre 0 e 2. Lembramos que (6.6) decai como uma lei de potência no limite de tempos longos $n \rightarrow \infty$

$$
G \sim \alpha(\alpha-1) n^{-\gamma}
$$

sendo $\gamma=2-\alpha$ ( $\gamma$ varia entre 0 e 2$)$ o expoente de decaimento.

Nas próximas seções discutiremos as predições relativas ao comportamento crítico da equação logística com desordem temporal correlacionada a partir mapeamento no movimento Browniano fracionário refletido. Iniciaremos discutindo o caso descorrelacionado, onde é possível obter a solução exata da densidade de probabilidade de $x=-\ln \rho$ a partir da equação de difusão, e em seguida faremos a análise do caso correlacionado, onde veremos como a singularidade em lei de potência na densidade de probabilidade (5.16) afeta as propriedades críticas da equação logística.

\subsection{Caso descorrelacionado $\gamma=\alpha=1$}

Nesta seção resumiremos os resultados da referência [87] a respeito da equação logística com desordem temporal descorrelacionada, a partir do mapeamento no passeio aleatório.

No capítulo 5 vimos que o movimento Browniano refletido apresenta três regimes de acordo com a velocidade de drift $v$ : (i) o regime localizado $(v<0)$, onde a posição média dos caminhantes tende a um valor finito $x_{\mathrm{st}}$, (ii) o regime balístico (se $v>0$ ), em que os caminhantes viajam com velocidade balística e $\langle x\rangle \rightarrow \infty$ se o tempo $t \rightarrow \infty$, (iii) a transição entre os dois regimes $(v=0)$, onde observamos difusão anômala $\langle x\rangle \sim t^{\alpha / 2}$. Como estes três regimes se relacionam com a equação logística? Usando o mapeamento $x=-\ln \rho$, podemos concluir que: (i) o regime localizado é equivalente à fase de densidade $\rho>0$ (sobrevivência ou fase ativa), (ii) o regime balístico corresponde à fase onde a população decai exponencialmente (extinção ou fase inativa), e (iii) a transição 
de regimes (ponto crítico) entre a extinção e a sobrevivência da população ocorre no regime de difusão anômala.

Feito a análise qualitativa dos diferentes regimes, estudaremos a densidade de probabilidade da posição $x$ de um passeio aleatório refletido (5.3) a fim de extrair as propriedades críticas da equação logística com desordem temporal. Na fase ativa (fase localizada), a densidade de probabilidade (5.3) tende ao estado estacionário

$$
P_{\text {st }}(x)=\frac{1}{2|v| \sigma^{2}} \exp \left\{\frac{-2|v| x}{\sigma^{2}}\right\} .
$$

Usando a equação acima, podemos calcular a densidade estacionária de indivíduos

$$
\langle\rho\rangle=\int_{0}^{\infty} e^{-x} P(x) d x \sim|v|,
$$

e a densidade típica de indivíduos

$$
\ln \rho_{\text {typ }}=-\langle x\rangle \sim|v|^{-1} .
$$

A diferença drástica entre o comportamento da densidade média e típica se deve ao comportamento singular da densidade de probabilidade, que pode ser melhor visualizada se escrita em função de $\rho$

$$
P(\rho) \sim|v| \rho^{-1+2|v| / \sigma^{2}} .
$$

No ponto crítico $(v=0)$ a densidade de probabilidade é uma meia gaussiana

$$
\frac{2}{\sqrt{2 \pi \sigma^{2} t}} \exp \left\{\frac{-x^{2}}{2 \sigma^{2} t}\right\},
$$

e a densidade média decai como uma lei de potência

$$
\langle\rho\rangle \sim t^{-1 / 2},
$$

enquanto que a densidade típica decai exponencialmente

$$
\ln \rho_{\text {typ }} \sim t^{-1 / 2} .
$$

$\mathrm{Na}$ fase inativa $(v>0)$ a densidade de probabilidade se move para valores grandes de $x$ e as duas densidades decaem exponencialmente com o tempo perto to ponto crítico

$$
\langle\rho\rangle \sim \exp \left\{-v^{2} t / 2 \sigma^{2}\right\}
$$


$\mathrm{e}$

$$
\rho_{\text {typ }} \sim \exp \{-v t\}
$$

O tempo de correlação temporal $\xi_{t}$ pode ser estimado como o tempo em que a crítica densidade média leva para desviar significativamente da densidade fora do ponto crítico. Comparando as equações (6.9) e (6.13), concluímos que

$$
\xi_{t} \sim|v|^{-2} .
$$

Se $|v|$ é a distância ao ponto crítico, podemos comparar as equações (6.9), (6.13) e (6.17) com o comportamento esperado próximo ao ponto crítico, isto é, $\rho \sim|v|^{\beta^{\text {unc }}}$, $\rho \sim t^{-\delta^{\text {unc }}}$, e $\xi_{t} \sim|v|^{-\nu_{\|}^{\text {unc }}}$. Dessa comparação obtemos os expoentes críticos

$$
\beta^{\mathrm{unc}}=1, \quad \delta^{\mathrm{unc}}=1 / 2 \quad \text { e } \quad \nu_{\|}^{\mathrm{unc}}=2 .
$$

Os expoentes acima diferem dos da equação logística sem desordem temporal ( $\beta^{\text {clean }}=1$, $\delta^{\text {clean }}=1$ e $\left.\nu_{\|}^{\text {clean }}=1[88]\right)$.

A equação logística não contém noção de um tamanho da população. No entanto, podemos introduzir um tamanho do sistema $N$ e definir que a população se torna extinta se $\rho_{n}<1 / N$. Sendo assim, podemos definir o tempo de vida $\tau$ da população como o tempo típico que a população leva para chegar em $\rho_{n}=1 / N$

$$
\tau^{-1} \sim \int_{0}^{1 / N} d \rho P(\rho)
$$

Logo, na fase ativa, temos

$$
\tau \sim N^{2|v| / \sigma^{2}}
$$

no ponto crítico

$$
\tau \sim \ln ^{2}(N)
$$

e na fase de extinção

$$
\tau \sim \ln N .
$$

O comportamento da tempo de sobrevivência está em concordância com a noção de fase de Griffiths temporal [89].

Além da solução através do mapeamento no passeio aleatório, Vojta e Hoyos também desenvolveram um grupo de renormalização para a equação logística com desordem temporal. A solução segue o grupo de renormalização de desordem forte [32, 33, 34, 35], e foi demonstrado que o comportamento crítico da equação logística com desordem 
temporal é idêntico ao do processo de contato com desordem espacial congelada, porém pela troca do espaço pelo tempo. De forma análoga ao ponto crítico de desordem infinita observado processo de contato com desordem congelada, o ponto crítico da equação logística com desordem temporal foi denominado ponto crítico de ruído infinito [87] (veja o apêndice B para um cálculo resumido da renormalização da equação logística com desordem temporal).

Assim como o ponto crítico de desordem infinita é caracterizado pelo crescimento sem limites da largura densidade de probabilidade das taxas, o ponto crítico de ruído infinito é também caracterizado pelo crescimento da largura da densidade de probabilidade de $\rho$, conforme (6.12). Além disso, o ponto crítico de desordem infinita apresenta uma relação logarítmica entre o comprimento de correlação espacial e temporal $\xi \sim\left(\ln \xi_{t}\right)^{1 / \psi}$ (activated scaling). Podemos encontrar uma relação análoga no ponto crítico de ruído infinito? Partindo do tempo de sobrevivência no ponto crítico (6.21) e definindo um comprimento de correlação espacial $\xi=N^{1 / d}$, obtemos

$$
\xi_{t} \sim(\ln \xi)^{1 / \omega}
$$

$\operatorname{com} \omega=1 / 2$.

\subsection{Teoria da equação logística com desordem temporal correlacionada}

Na seção anterior analisamos o comportamento crítico da equação logística com desordem temporal descorrelacionada através do mapeamento no passeio aleatório. Na presença de um ruído correlacionado, a equação logística pode ser mapeada no movimento Browniano fracionário refletido. A seguir apresentaremos a teoria do comportamento crítico da equação logística com desordem temporal correlacionada [90], com base nos resultados obtidos para o movimento Browniano fracionário refletido.

\subsubsection{Critério de Harris}

Primeiramente é interessante saber se a desordem é relevante no ponto crítico. Para isso, aplicaremos a generalização espaço-temporal do critério de Harris [91] para desordem temporal com correlações que decaem conforme uma lei de potência. Este critério afirma que se

$$
\gamma \nu_{\|}>2
$$


então o comportamento crítico é estável na presença da desordem temporal correlacionada (veja a seção 2.4).

Aplicando este critério na equação logística sem desordem $\left(\nu_{\|}^{\text {clean }}=1\right)$, vemos que todos os valores possíveis de $\gamma(0<\gamma<2)$ desestabilizam o comportamento da equação logística sem desordem. Portanto, esperamos que a desordem correlacionada mude o comportamento crítico da equação logística.

\subsubsection{Comportamento crítico}

Nesta subseção deduziremos as propriedades críticas da equação logística com desordem temporal correlacionada. Os resultados serão deduzidos a partir do estudo do movimento Browniano fracionário refletido 5 , com destaque para a singularidade em forma de lei de potência (5.16) observada na densidade de probabilidade da posição $x=-\ln \rho$ do caminhante.

Vimos anteriormente que a densidade de probabilidade da posição do caminhante segue a forma (5.15), e que no ponto crítico há uma singularidade em lei de potência $P \sim x^{\kappa}$ (5.16). Como $\rho=e^{-x}$, a principal contribuição para a média da densidade de indivíduos será devida à singularidade na densidade de probabilidade. Usando as equações (5.15) e (5.16), a densidade média de indivíduos no ponto crítico será dada por

$$
\langle\rho\rangle=\int e^{-x} P(x, t) d x \sim \int e^{-x} \frac{d x}{t^{\alpha / 2}}\left(\frac{x}{t^{\alpha / 2}}\right)^{\kappa} \sim t^{-\gamma / 2},
$$

onde $\gamma=2-\alpha$. Em contraste, a densidade típica $\rho_{\text {typ }}=e^{-\langle x\rangle}$ decai muito mais rapidamente

$$
\rho_{\text {typ }} \sim \exp \left\{-D t^{\phi}\right\}
$$

onde $D$ é uma constante e

$$
\phi=(2-\gamma) / 2
$$

O tempo de sobrevivência de uma população é o tempo que a densidade típica leva para cair abaixo de $1 / N$. Usando a equação (6.26) encontramos

$$
\tau \sim(\ln N)^{\eta}
$$

com

$$
\eta=\frac{2}{2-\gamma} .
$$

O tempo de correlação temporal $\xi_{t}$ pode ser deduzido através do mesmo procedi- 
mento usado no movimento Browniano fracionário refletido. $\xi_{t}$ é o tempo em que a posição do caminhante no regime balístico $(v t)$ desvia significativamente do comportamento observado no ponto crítico $\left(t^{(2-\gamma) / 2}\right)$

$$
\xi_{t} \sim|v|^{-2 / \gamma}
$$

A densidade estacionária na fase ativa pode ser estimada como a densidade no tempo $\xi_{t}$. Usando (6.25) e (6.30), encontramos

$$
\langle\rho\rangle \sim|v|^{1}
$$

A partir das equações (6.25), (6.30) e (6.31), podemos encontrar os expoentes críticos

$$
\delta=\gamma / 2, \quad \nu_{\|}=2 / \gamma \quad \text { e } \quad \beta=1 .
$$

Se $\gamma=1$ (ruído descorrelacionado), os expoentes acima concordam com os expoentes críticos do caso descorrelacionado (6.18). Além disso, os expoentes seguem a relação de escala $\delta=\beta / \nu_{\|}(2.7)$.

Apesar da equação logística não ter noção de comprimento, podemos introduzir o comprimento do sistema através da relação $\xi^{d} \sim N$. A partir desta noção de comprimento e da equação (6.28), obtemos

$$
\xi_{t}^{(2-\gamma) / 2} \sim \ln \xi
$$

Assim como no caso de ruído descorrelacionado, essa é a versão temporal do escalamento ativo observada no caso do processo de contato com desordem espacial congelada. O expoente $\omega=(2-\gamma) / 2$ é a versão temporal do expoente de tunelamento $\psi$.

\subsubsection{Fase de Griffiths temporal}

A fase de Griffiths temporal é a versão temporal da fase de Griffiths (espacial). Enquanto que a fase de Griffiths (espacial) ocorre na fase inativa [92, 93], a fase de Griffiths temporal é observada quando o sistema está na fase ativa [89]. Especificamente, a fase de Griffiths temporal ocorre devido à intervalos de tempo raros que fazem com que o sistema aparente estar na fase inativa, enquanto que o bulk to sistema está na fase ativa.

Seguindo este raciocínio, podemos investigar a fase Griffiths temporal de forma similar ao estudo da cauda da densidade de probabilidade e do tempo de primeira 
passagem no movimento Browniano fracionário 5.3.3. Considerando o ruído apenas na taxa $\mu(\Delta \lambda=0)$, a probabilidade de encontrar um intervalo de tempo de tamanho $T_{\mathrm{RR}}$ e ruído efetivo

$$
\mu_{\mathrm{RR}}=\frac{1}{T_{\mathrm{RR}}} \sum_{i \in \mathrm{RR}} \mu_{i}
$$

é dada por

$$
P \sim \exp \left\{-A T_{\mathrm{RR}}^{\gamma}\right\},
$$

sendo que $A$ é uma constante. Para que haja a extinção da população no tempo $T_{\mathrm{R} R}$, o ruído efetivo deve ser forte o suficiente para fazer a densidade de indivíduos cair abaixo de $1 / N$, ou seja

$$
a_{i+1} a_{i+2} \ldots a_{i+T_{R R}}=e^{\left(\lambda-\mu_{R R}\right) T_{R R}} \sim 1 / N .
$$

Sendo assim, concluímos que $T_{\mathrm{RR}}=(\ln N) /\left(\mu_{\mathrm{RR}}-\lambda\right)$. Substituindo a expressão para $T_{\mathrm{RR}}$ em (6.35), e levando em conta que o tempo de sobrevivência da população é proporcional ao inverso de $P$, obtemos

$$
\tau \sim \exp \left\{C(\ln N)^{\gamma}\right\}
$$

Enquanto que a equação (6.37) nos dá a dependência de $\tau$ com $N$, esta derivação não é suficiente para entender a dependência do prefator $C$ com $v$. No entanto, a dependência de $C$ com $v$ pode ser achada usando argumentos de escalamento. Devido à simplicidade da equação logística, as únicas variáveis relevantes são o tamanho da população e a distância ao ponto crítico $|v|$. Combinando as equações (6.28) e (6.30), encontramos

$$
(\ln N)^{-\gamma} \sim|v|^{2-\gamma} .
$$

Usando a equação acima e o tempo de sobrevivência no ponto crítico (6.28) podemos inferir

$$
\tau=(\ln N)^{2 /(2-\gamma)} \Phi\left(|v|^{(2-\gamma)}(\ln N)^{\gamma}\right) .
$$

Comparando as equações (6.37) e (6.39), concluímos que

$$
\tau \sim \exp \left\{C^{\prime}|v|^{2-\gamma}(\ln N)^{\gamma}\right\} .
$$

\subsection{Simulações}

Nesta seção apresentaremos as simulações feitas para confirmar a teoria. As simulações consistem em iterar a equação (6.3) a fim de obter a evolução da densidade de indivíduos. 


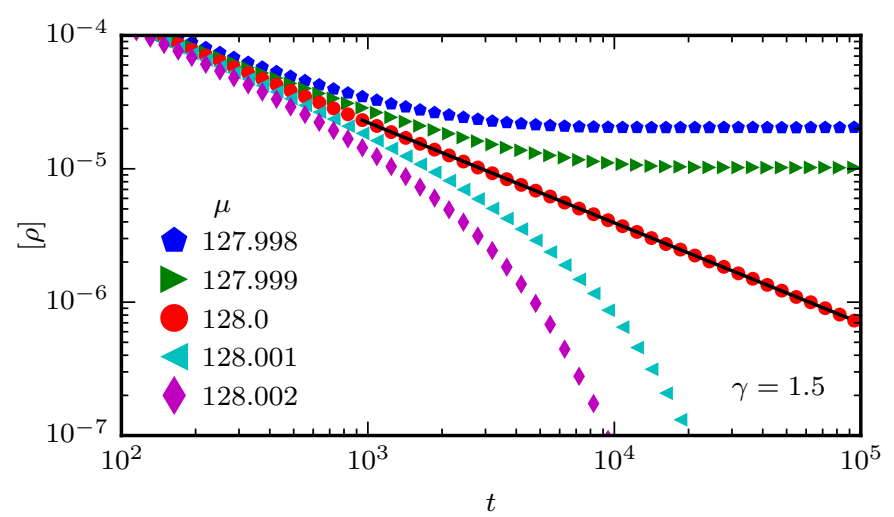

Figura 6.2: Comportamento qualitativo da densidade média $\langle\rho\rangle$ perto to ponto crítico $\mu_{c}=\lambda=128$ para $\gamma=1.5$. As incertezas são da ordem do tamanho dos pontos.

Os parâmetros relevantes são as taxas médias $\mu$ e $\lambda$, os ruídos $\Delta \mu_{n}$ e $\Delta \lambda_{n}$, e intervalo de tempo $\Delta t$.

Note que a evolução da densidade (6.3) é invariante pelas transformações $\lambda_{n} \rightarrow M \lambda_{n}$, $\mu_{n} \rightarrow M \mu_{n}$ e $\Delta t \rightarrow M^{-1} \Delta t$. Logo podemos usar esta transformação para escolher parâmetros convenientes.

Por simplicidade, a desordem temporal será inclusa apenas na variável $\Delta \mu_{n}$. Especificamente, $\Delta \mu_{n}$ será uma variável aleatória Gaussiana com variância $\sigma^{2}$, média nula e correlações de longo alcance, enquanto que $\Delta \lambda=0$. Além disso, fixaremos a taxa média de crescimento em $\lambda=128$, e usaremos $\mu$ para controlar a distância ao ponto crítico $|v|=\mu-\lambda$. Isso implica que a transição de fase ocorrerá em $\mu_{c}=\lambda=128$, a fase ativa (sobrevivência) ocorre se $v=\mu-\mu_{c}<0$, e temos a extinção da população se $v=\mu-\mu_{c}>0$.

Todas as simulações começaram de $\rho_{0}=1$ (ou $x=0$ ) e chegam a um tempo máximo de $2^{26}\left(6.7 \times 10^{7}\right)$ iterações. A variância do ruído $\Delta \mu_{n}$ será $\sigma^{2}=1$ e $\Delta t=2$, a menos outro valor seja explicitamente mencionado.

\subsubsection{Comportamento crítico}

Primeiramente verificaremos o comportamento qualitativo quando $\mu<\mu_{c}, \mu=\mu_{c}$ e $\mu>\mu_{c}$. A figura 6.2 mostra a densidade média $\langle\rho\rangle$ em função do tempo $t$ para $\gamma=1.5$ (correlações negativas) e diversos valores de $v$. Conforme esperado, $\langle\rho\rangle$ apresenta o seguinte comportamento: se $\mu<\mu_{c}$ a população sobrevive e $\langle\rho\rangle$ alcança um valor estacionário, se $\mu=\mu_{c}$ temos o decaimento da densidade conforme uma lei de potência, e se $\mu>\mu_{c}$ a densidade média decai mais rapidamente que uma lei de potência. 
Após verificar o comportamento qualitativo da densidade média, é importante verificar se a singularidade em lei de potência também existe na equação logística. Na figura 6.3 podemos ver a densidade de probabilidade de $x=-\ln \rho$ para os ruído com correlações positivas, descorrelacionado e negativas, nos painéis (a), (c) e (e), respectivamente. Se $\gamma=0.5$ ou 1.5, podemos ver que $P$ diverge e tende a zero, respectivamente, conforme esperado da singularidade. Além disso, os painéis (b) e (e) mostram que esta singularidade é compatível com uma lei de potência. Se o ruído for descorrelacionado, $P$ é compatível com a meia gaussiana (linha sólida) $P(x, t)=2 \exp \left\{-x^{2} /\left(2 \sigma^{2} \Delta t^{2} t\right)\right\} / \sqrt{2 \pi \sigma^{2} \Delta t^{2} t}$, que é solução da equação de difusão com condição de fluxo nulo em $x=0$.

Agora analisaremos a densidade típica e média no ponto crítico. Na figura 6.4 vemos o logaritmo da densidade típica $\langle x\rangle=-\ln \rho_{\text {typ }}$ e a densidade média $\langle\rho\rangle$ em função do tempo, para vários valores do expoente de correlação $\gamma$ em escala log-log. Nestes gráficos podemos ver que ambas as grandezas seguem o comportamento em lei de potência $\langle x\rangle \sim t^{\phi}(6.26)$ e $\langle\rho\rangle \sim t^{-\delta}(6.25)$. Para verificar se $\phi=(2-\gamma) / 2$ e $\delta=2 / \gamma$, ajustamos leis de potências nos dados (linhas sólidas) e plotamos os expoentes ajustados na figura 6.5. Nesta figura podemos ver que os expoentes ajustados concordam bem com as expectativas. Perceba, no entanto, que há desvios sistemáticos no expoente $\phi$ para valores de $\gamma>1$. Estes desvios também são observados no movimento Browniano fracionário e estão relacionados com o longo crossover no regime subdifusivo, pois a densidade de probabilidade demora muito mais para se alargar. Por fim, a figura 6.4(c) mostra que as densidades típica e média se escalam de forma bastante diferente com o tempo.

Para finalizar o estudo do ponto crítico, mostramos os resultados das simulações para o tempo de sobrevivência da população $\tau$. Na figura 6.6(a) plotamos $\tau$ vs. $\ln N$, para diversos valores de $\gamma$ e fixando a largura do ruído $\Delta \mu_{n}$ em $\sigma=2$, de forma que o comportamento predito $\tau \sim(\ln N)^{\eta}$ (6.28) seja visualmente linear. Neste gráfico podemos ver que os resultados das simulações concordam muito bem com (6.28).

Antes de efetuar ajustes da função $\tau \sim(\ln N)^{\eta}$, a fim de verificar se os dados são compatíveis com o expoente $\eta=2 /(2-\gamma)$, percebemos que o crossover para o comportamento assintótico muda significativamente se mudarmos $\sigma$, segundo a figura 6.6(b). Para valores de $\sigma$ pequenos, o sistema leva muito tempo para desviar do comportamento da equação logística sem desordem, pois $\sigma=0$ corresponde ao caso de ruído nulo. Por outro lado, quando $\sigma$ é muito grande, a população tende à extinção em uma única iteração, fazendo com que o comportamento assintótico seja arrastado para valores maiores de $\ln N$.

Para avaliar o melhor valor de $\sigma$, levamos em consideração apenas os ajustes cujo 

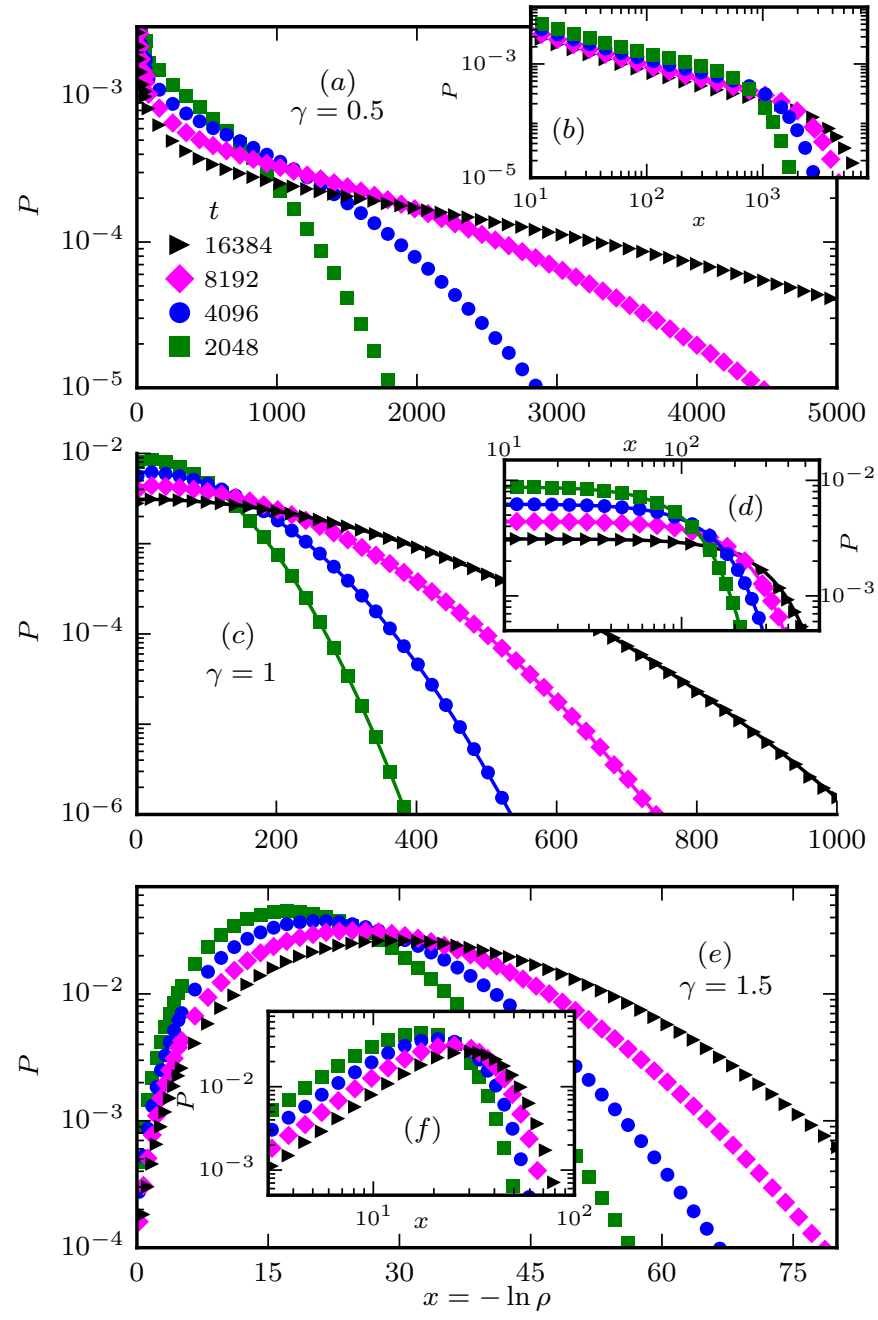

Figura 6.3: Densidade de probabilidade $P$ em função da posição $x=-\ln \rho$. Os painéis $(\mathrm{a}, \mathrm{b}),(\mathrm{c}, \mathrm{d})$ e $(\mathrm{e}, \mathrm{f})$ mostram os casos de correlações positivas $(\gamma<1)$, descorrelacionado $(\gamma=1)$ e negativas $(\gamma>1)$. As incertezas são da ordem do tamanho dos pontos. 


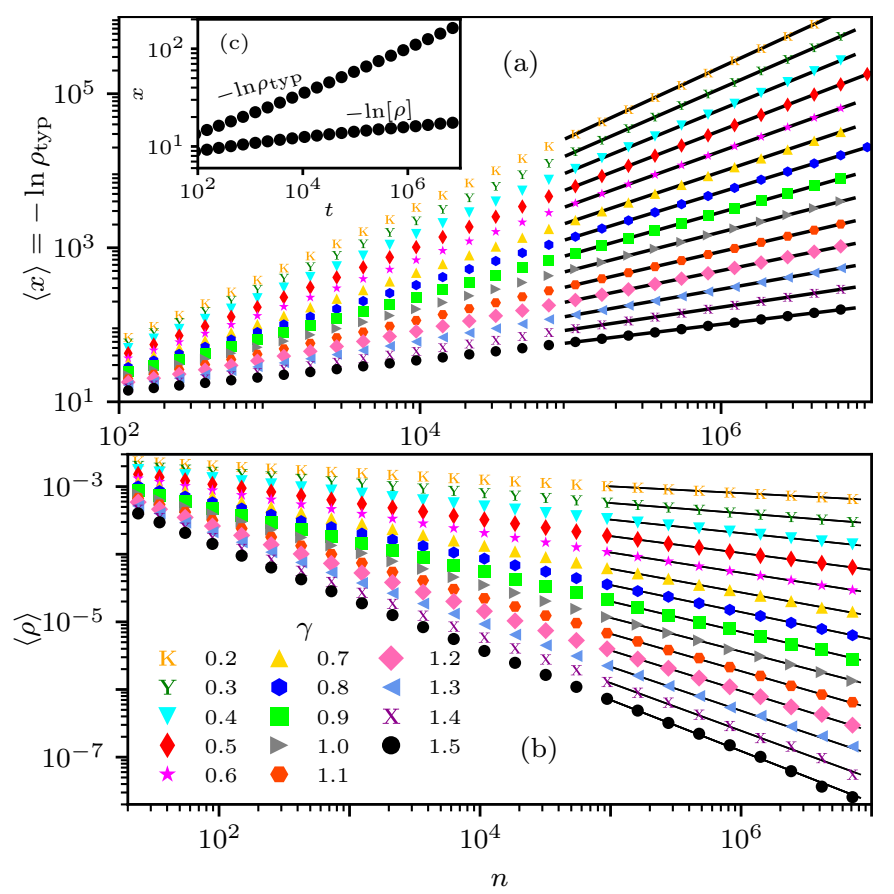

Figura 6.4: (a) Logaritmo da densidade típica $\langle x\rangle=-\ln \rho_{\text {typ }}$ e (b) densidade média $\langle\rho\rangle$ vs. tempo $t$ no ponto crítico $\mu=\mu_{c}=\lambda=128$ para diversos valores de $\gamma$. As linhas sólidas são ajustes das leis de potência $\langle x\rangle \sim t^{\phi}(6.26)$ e $\langle\rho\rangle \sim t^{-\delta}$ (6.25). As incertezas são menores que o tamanho dos pontos.

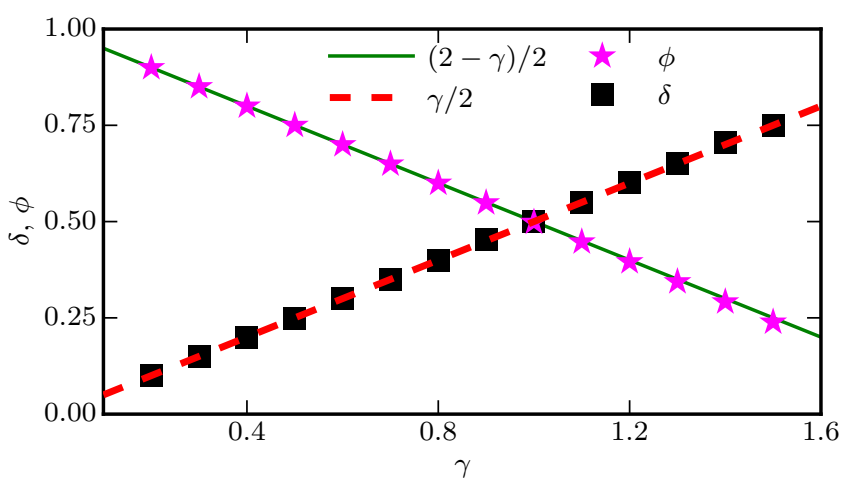

Figura 6.5: Expoentes críticos $\phi$ e $\delta$ extraídos dos dados da figura 6.4. A linha sólida e tracejada representam as predições $\phi=(2-\gamma) / 2(6.27)$ e $\delta=\gamma / 2(6.32)$. As incertezas são menores que o tamanho dos pontos. 

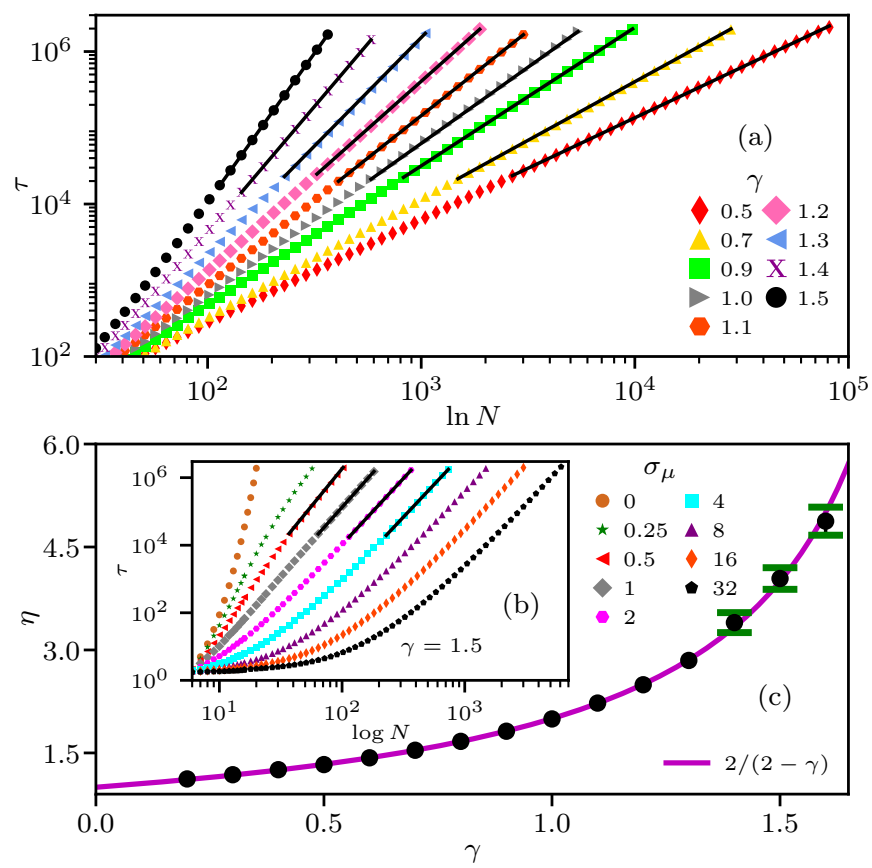

Figura 6.6: (a) Tempo de sobrevivência $\tau$ vs. logaritmo do tamanho da população $\ln N$ no ponto crítico para $\sigma=2$ e vários valores de $\gamma$. (b) $\tau$ vs. $\ln N$ para $\gamma=1.5$ e diversos valores de $\sigma$. As linhas sólidas são ajustes da forma $\tau \sim[\ln N]^{\eta}$ (6.28). (c) Expoentes $\tau$ ajustados vs. $\gamma$. A linha sólida mostra a predição $\eta=2 /(2-\gamma)$ (6.29). As incertezas, se não explicitamente plotadas, são menores que o tamanho dos pontos.

chi-quadrado reduzido $\chi^{2}<1.5$. Apesar dos valores ideias de $\sigma$ mudarem um pouco com $\gamma$, valores de $\sigma$ entre 0.5 e 4 geram bons resultados.

Seguindo este procedimento para diversos valores de $\gamma$, obtemos os expoentes plotados em 6.6(c). Neste gráfico podemos ver que os expoentes concordam muito bem com $\eta=2 /(2-\gamma)$. As incertezas são oriundas da variação dos expoentes com $\sigma$, e são muito menores que o tamanho do símbolo se não plotadas.

\subsubsection{Fora do ponto crítico}

A figura 6.7(a) mostra as simulações para a densidade média na fase ativa para $\gamma=1.5$. A densidade estacionária $\rho_{\text {st }}$ é estimada ajustando uma constante no comportamento assintótico de $\langle\rho\rangle$. O painel (b) mostra a densidade estacionária vs. $|v|=\left|\mu-\mu_{c}\right|$ para diversos valores de $\gamma$. Como a densidade estacionária é compatível com leis de potência para todos os valores de $\gamma$ plotados, realizamos ajustes de leis de potência para extrair o expoente crítico $\beta$. Os expoentes ajustados estão plotados na figura 6.8 , onde podemos 

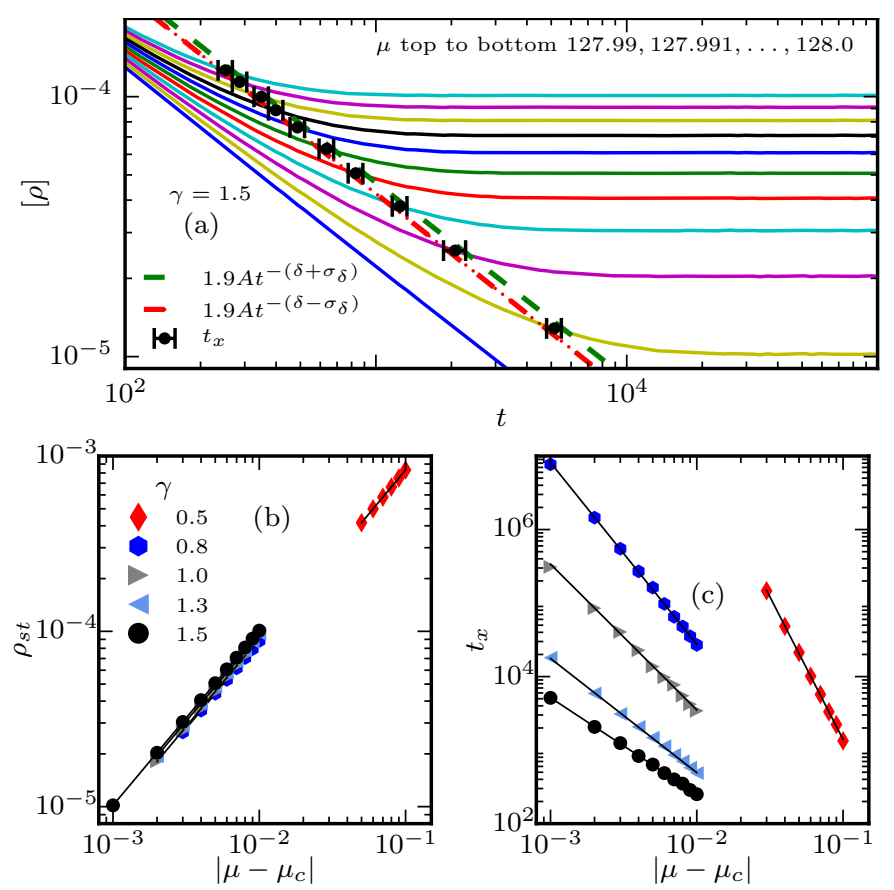

Figura 6.7: (a) Densidade média $\langle\rho\rangle$ vs. tempo $t$ na fase ativa $\mu<\mu_{c}$ para $\gamma=1.5$ e diversos valores de $\mu$ (linhas sólidas). As linhas tracejadas representam $1.9 \times \rho_{\text {crit }}$, onde $\rho_{\text {crit }}=A t^{-\delta}$ é um ajuste da curva no ponto crítico $\mu=\mu_{c}$. Os círculos pretos são a estimativa do tempo de correlação $t_{x}$. (b) Densidade estacionária vs. distância ao ponto crítico $\left|\mu-\mu_{c}\right|$ para diversos valores de $\gamma$. (c) Tempo de correlação $t_{x}$ vs. $\left|\mu-\mu_{c}\right|$ para os mesmos valores de $\gamma$ encontrados no painel (b). As incertezas são da ordem da espessura das linhas ou do tamanho dos pontos.

observar uma boa concordância com o valor teórico $\beta=1$.

O tempo de correlação $\xi_{t}$ pode ser estimado como o tempo $t_{x}$ em que a densidade média desvia de $1.9 \times \rho_{\text {crit }}$ (linha sólida na figura 6.7 ), onde $\rho_{\text {crit }}$ se escala com $t^{-\delta}$ e delta é o expoente ajustado no ponto crítico. Os valores estimados de $t_{x}$ estão na figura 6.7(c). Assim como no caso de $\rho_{\mathrm{st}}, t_{x}$ seguem leis de potência em $|v|=\left|\mu-\mu_{c}\right|$. Os valores dos expoentes ajustados estão plotados na figura 6.8 , onde podemos ver uma ótima concordância com a predição $t_{x} \sim|v|^{-\nu_{\|}}$(6.30), com $\nu_{\|}=2 / \gamma$.

\subsubsection{Fase de Griffiths temporal}

A fase de Griffiths temporal ocorre na fase ativa $(\mu-\lambda>0)$ devido a intervalos de tempo raros onde o ruído $\left(\Delta \mu_{n}\right)$ faz com que com que o processo esteja localmente na fase inativa (inativa). Portanto, para simular a fase de Griffiths temporal, a desordem 


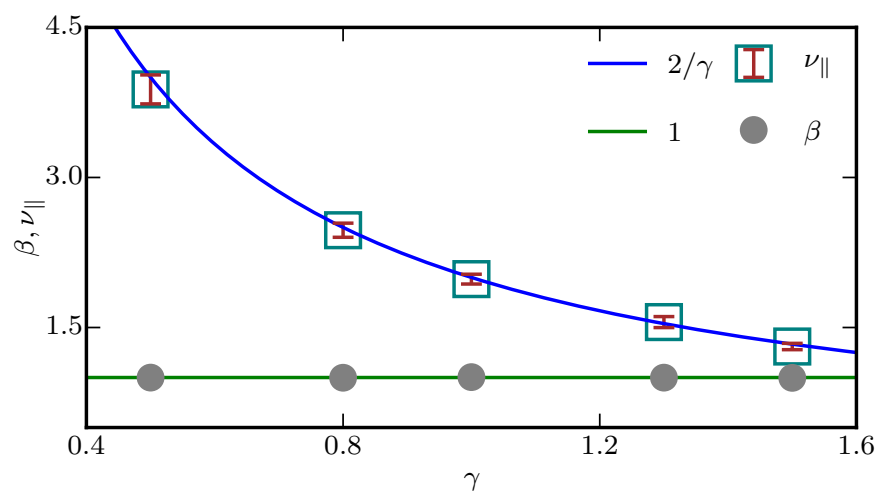

Figura 6.8: Expoentes críticos $\beta$ e $\nu_{\|}$extraídos dos dados plotados nos painéis (b) e (c) da figura 6.7. As linhas sólidas indicam as predições $\beta=1$ e $\nu_{\|}=2 / \gamma(6.32)$. A incerteza em $\beta$ é da ordem de $10^{-2}$.

temporal deve se capaz de produzir taxas $\mu_{n}$ na fase inativa mesmo que o sistema esteja na fase ativa. Dessa forma, as simulações feitas nesta seção serão feitas com uma distância máxima de $2 \sigma$ do ponto crítico, implicando uma probabilidade mínima de aproximadamente $2.5 \%$ de encontrar $\mu_{n}$ na fase inativa.

A figura 6.9 mostra $\ln \tau$ vs. $(\ln N)^{\gamma}$ para $\gamma=1.5$ e 0.5 e vários valores de $\mu$. Nesta figura podemos ver que $\tau$ segue o comportamento assintótico esperado $\ln \tau=C(\ln N)^{\gamma}$ (6.37) por três décadas, se $\gamma=1.5$, e uma década se $\gamma=0.5$, portanto efetuaremos ajustes para verificar se $C$ se escala com $\left|\mu-\mu_{c}\right|^{2-\gamma}$.

As figuras 6.10(a) e 6.10(b) mostram os valores ajustados de $C$ vs. $\left|\mu-\mu_{c}\right|$. Os dados são compatíveis com leis de potência, porém vemos desvios desse comportamento. Os desvios podem ser entendidos da seguinte forma: longe do ponto crítico as regiões raras ocorrem com menor frequência, logo precisamos de um tempo maior de simulação para alcançar o regime assintótico, perto do ponto crítico a velocidade de bias (6.5) é menor e leva mais tempo para que a densidade média se diferencie da densidade crítica.

Descartando valores de $C$ perto e longe do ponto crítico e ajustando leis de potência nos dados restantes, encontramos os valores plotados em 6.10(c). Neste gráfico podemos ver que os expoentes ajustados concordam bem com a predição.

É possível colapsar $\tau$ de acordo com (6.39)? Apesar de $\tau$ seguir o comportamento predito, não conseguimos colapsar $\tau$ com usando $\sigma=1$ e $\Delta t=2$. No entanto, usando os parâmetros $\sigma=32, \Delta t=10^{32}$ e $\gamma=1.5^{1}$, obtemos o colapso quase perfeito plotado na figura 6.11. No eixo $y, \tau_{0}(\mu)$ age como uma correlação de tempos finitos e foi obtida

\footnotetext{
${ }^{1}$ Perceba que estes parâmetros correspondem a uma desordem extremamente forte e não tem sentido prático, pois causam a mudanças na densidade de indivíduos da ordem de $e^{ \pm 32 \times 10^{32}}$
} 

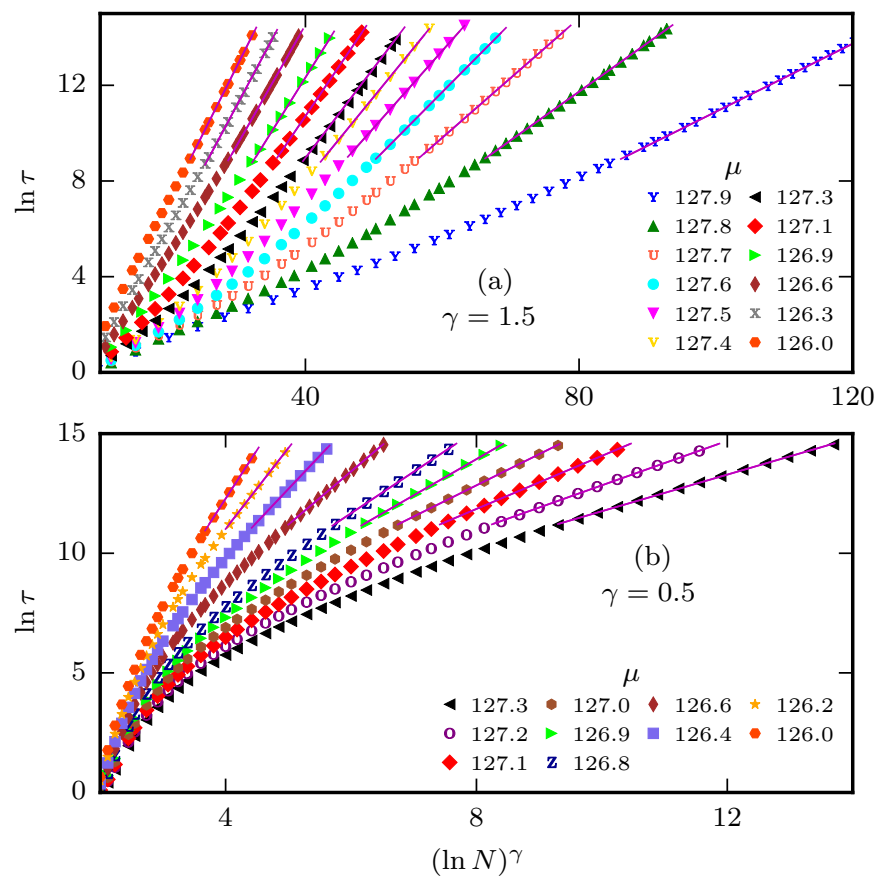

Figura 6.9: Logaritmo do tempo de sobrevivência vs. $(\ln N)^{\gamma}$ de forma que o comportamento predito (6.37) seja visualmente linear. As linhas retas representam ajustes de $\ln \tau=C(\ln \tau)^{\gamma}+B$. O prefator $C$ está plotado na figura 6.10. As incertezas são menores do que o tamanho dos pontos. 

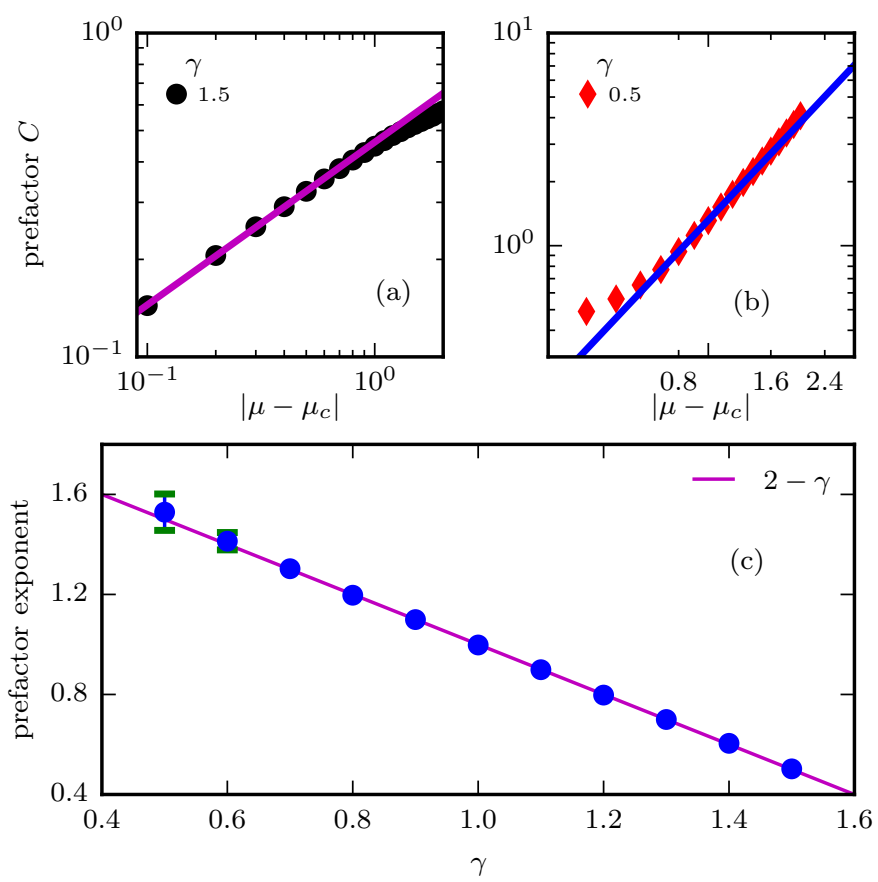

Figura 6.10: (a,b) Prefator $C$ ajustado nos dados da figura 6.9 de acordo com a predição (6.37). A linha sólida representa um ajuste da lei de potência $C \operatorname{sim}|v|^{2-\gamma}$. (c) Expoente do prefator para diversos valores de $\gamma$. A linha sólida mostra a predição $2-\gamma(6.40)$. As incertezas são menores que o tamanho dos pontos se não plotadas. 


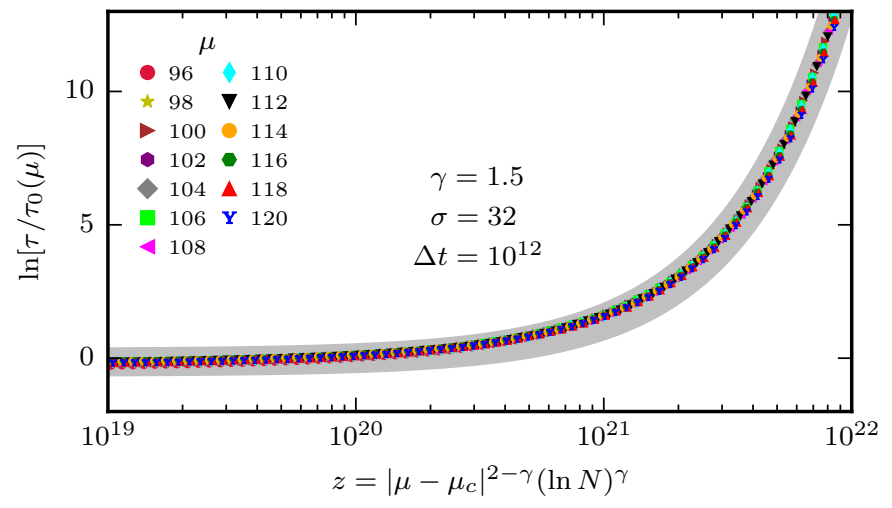

Figura 6.11: Colapso dos tempo de sobrevivência de acordo com (6.39) para $\gamma=1.5$. Neste plote usamos uma desordem extremamente forte com parâmetros $\sigma=32$ e $\Delta t=$ $10^{12}$. A correlação à escala $\tau_{0}(\mu)$ foi obtida ajustando a função $\tau \sim \tau_{0}(\mu) e^{C\left|\mu-\mu_{c}\right|^{2-\gamma}[\ln N]^{\gamma}}$ em cada uma das curvas. A tira cinza representa $\tau=D_{1} e^{D_{2} z}$, onde $D_{1}$ e $D_{2}$ são constantes com os valores apropriados.

através ajustando a função $\tau_{0}(\mu) e^{C^{\prime}\left(\mu-\mu_{c}\right)^{2-\gamma}[\ln N]^{\gamma}}$. 



\section{Capítulo 7}

\section{Conclusão}

Neste trabalho estudamos os efeitos de desordem espacial e temporal em modelos prototípicos para a dinâmica de populações biológicas, por meio de simulações de Monte Carlo e argumentos de escala. Enquanto que o caso de desordem espacial se manifestou indiretamente no modelo epidemiológico de quatro estados (modelo EQE), introduzimos desordem temporal na equação logística permitindo que as taxas de morte variem no tempo.

O modelo EQE possui quatro estados: suscetível, imune, exposto e infectado. Através de uma escolha particular dos parâmetros, vimos que o modelo EQE pode ser mapeado exatamente no processo de contato com diluição. Neste mapeamento os sítios imunes, expostos e infectados correspondem a sítios vazios, ocupados e infectados, respectivamente. Este mapeamento ressalta a importância do estudo de sistemas com desordem, pois mostra que a desordem pode surgir naturalmente em modelos mais complexos.

Para confirmar esta hipótese, realizamos simulações do modelo EQE em duas dimensões, onde os expoentes críticos do processo de contato com diluição já foram calculados numericamente com boa precisão. Como esperado, nossas simulações confirmaram que o modelo EQE tem os mesmos expoentes críticos do processo de contato com diluição. Além disso, devido ao mapeamento dos sítios imunes e expostos nos sítios vazios e ocupados da percolação, o modelo EQE também apresenta uma transição de fase na classe de universalidade da percolação dinâmica. Esta última transição foi verificada nas simulações em três dimensões, onde calculamos os expoentes críticos dinâmicos com maior precisão em relação aos valores encontrados na literatura.

Em seguida estudamos o movimento Browniano fracionário refletido, por meio de simulações de Monte Carlo. Vimos que o movimento Browniano fracionário refletido apresenta três regimes: o regime localizado, o regime balístico e a transição entre os 
regimes.

$\mathrm{Na}$ transição de fase vimos que, apesar da variância do caminhante ter o mesmo comportamento do caso não refletido (difusão anômala), o comportamento da densidade de probabilidade nos casos não confinado e refletido difere drasticamente. No caso não confinado, a densidade de probabilidade $P$ é gaussiana, ao passo que $P$ apresenta uma singularidade perto da parede refletora quando confinada. Esta singularidade se mostrou compatível com uma lei de potência $P \sim x^{\kappa}$. Através das simulações, pudemos conjecturar $\kappa=2 / \alpha-2$.

No regime localizado os caminhantes ficam próximos da parede refletora e a posição média alcança um valor finito no limite de tempos longos. Perto da parede refletora a densidade de probabilidade apresenta a mesma singularidade em lei de potência encontrada no ponto crítico. Longe da parede refletora a densidade de probabilidade se anula como uma exponencial esticada.

No regime balístico os caminhantes movem-se balisticamente para longe da parede refletora. No limite de tempos longos, a densidade de probabilidade tende à mesma gaussiana encontrada no caso não confinado.

Por fim, estudamos a equação logística com desordem temporal com correlações de longo alcance. Elaboramos a teoria do comportamento crítico deste problema através do mapeamento no movimento Browniano fracionário refletido. Neste teoria vimos como as correlações de longo alcance afetam o comportamento crítico do sistema. No estudo das propriedades críticas destacamos que o tempo de sobrevivência $\tau$ da população está em concordância com a noção de fase de Griffiths temporal [89] Todos os resultados foram verificados através de simulações de Monte Carlo. 


\section{Apêndice A}

\section{Grupo de renormalização de desordem forte}

O grupo de renormalização de desordem forte (Strong Disorder Renormalization Group

- SDRG), é um grupo de renormalização no espaço real (Real Space Renormalization Group), foi introduzido por Ma, Dasgupta e Hu [32, 33] e destacou-se após Fisher [34, 35] mostrar que esse grupo de renormalização é assintoticamente exato para o modelo de Ising no campo aleatório transverso (RTFI). Com essa técnica, reproduziremos a solução de Hooyberghs [94, 36, 37] para o RTFI e o processo de contato com desordem congelada, para mostrar um comportamento crítico temporal diferente de leis de potência.

O algoritmo consiste em selecionar iterativamente o termo mais forte da Hamiltoniana, digamos $\Omega$, e dizimá-lo através de um tratamento perturbativo. Neste processo eliminamos um grau de liberdade e introduzimos novas interações, supostamente mais fracas que as anteriores, abaixando a escala de energia. Se após muitas iterações a distribuição dos parâmetros alargar sem limites, o algoritmo torna-se assintoticamente exato, pois os resultados da teoria de perturbação ficam cada vez melhores dado que a larga distribuição torna cada vez mais improvável encontrar valores próximos a $\Omega$ na vizinhança do mesmo. 


\section{A.1 Solução do modelo de Ising aleatório no campo trans- verso unidimensional}

Considere o RTFI em uma dimensão, ilustrado na figura (A.1), cuja Hamiltoniana é dada por

$$
H=-\sum_{k=1}^{N} J_{k} \sigma_{k}^{x} \sigma_{k+1}^{x}-\sum_{k=1}^{N} h_{k} \sigma_{k}^{z},
$$

sendo que os acoplamentos e campos $\left(J_{k}\right.$ e $\left.h_{k}\right)$ seguem distribuições de probabilidades $R(J)$ e $P(h)$, e $\sigma$ são as matrizes de Pauli atuando no sítio $k$. Nas próximas seções, analisaremos o comportamento crítico desse sistema conforme [35, 94, 37]. Começaremos deduzindo as regras de dizimação que eliminará o termo mais forte da Hamiltoniana e depois escreveremos as equações de fluxo que nos permitirá resolver o problema perto do ponto fixo. Porém antes é importante comentarmos a repeito da dualidade deste modelo, pois esta propriedade facilitará a identificação do ponto crítico.

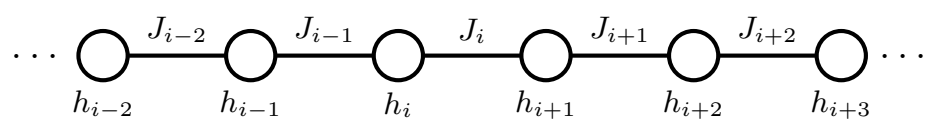

Figura A.1: Exemplo de cadeia unidimensional com acoplamentos $J_{i}$ e campos $h_{i}$ associados a cada sítio $i$.

Para observar a dualidade, basta aplicar as transformações

$$
\tau_{i+1 / 2}^{z}=\sigma_{i}^{x} \sigma_{i+1}^{x} \quad \text { e } \quad \tau_{i+1 / 2}^{x}=\prod_{i<j} \sigma_{j}^{z}
$$

em (A.1), obtendo uma equação idêntica

$$
H=-\sum_{k=1}^{N} J_{k} \tau_{i+1 / 2}^{z}-\sum_{k=1}^{N} h_{k} \tau_{i-1 / 2}^{x} \tau_{i+1 / 2}^{x}
$$

porém com o papel dos acoplamentos e campos trocados. Esta simetria permite observar que não há preferência entre campos e acoplamentos, logo é possível inferir que o ponto crítico está situado exatamente quando as distribuições de $J_{k}$ e $h_{k}, R(J)$ e $P(h)$, forem idênticas. 


\section{A.1.1 Dizimação do campo $h_{i}$}

Se a temperatura for baixa e

$$
H_{0}=-h_{i} \sigma_{i}^{z}
$$

for mais forte na Hamiltoniana, há uma grande probabilidade do sítio $i$ estar alinhado com o campo $h_{i}$. Portanto é razoável tratar o termo $H_{0}$ exatamente e os termos na vizinhança de $i$,

$$
V=J_{i-1} \sigma_{i-1}^{x} \sigma_{i}^{x}+J_{i} \sigma_{i}^{x} \sigma_{i+1}^{x},
$$

como uma perturbação. Sendo assim é conveniente reescrever (A.1)

$$
H=H_{0}-V-\sum_{\substack{k=1 \\ k \neq i-1, i}}^{N} J_{k} \sigma_{k}^{x} \sigma_{k+1}^{x}-\sum_{\substack{k=1 \\ k \neq i}}^{N} h_{k} \sigma_{k}^{z} .
$$

Para fazer usar a teoria de perturbação, precisamos dos autovalores e autovetores de (A.4), que são

$$
\lambda_{i}^{+}=-h_{1} \quad \text { e } \quad|+\rangle_{i}=\left(\begin{array}{l}
1 \\
0
\end{array}\right)
$$

e

$$
\lambda_{i}^{-}=h_{1} \quad \text { e } \quad|-\rangle_{i}=\left(\begin{array}{l}
0 \\
1
\end{array}\right),
$$

de forma que podemos representar o autovalor e estado mais provável por

$$
\lambda_{i}=-\left|h_{i}\right| \quad \text { e } \quad\left|\operatorname{sign}\left(h_{i}\right)\right\rangle_{i} .
$$

Definido o estado do sítio $i$ conforme (A.9), podemos usar a teoria de perturbação em $H_{0}-V$ até a segunda ordem

$$
H_{0}-V \approx-\left|h_{i}\right|-{ }_{i}\left\langle\operatorname{sign}\left(h_{i}\right)|V| \operatorname{sign}\left(h_{i}\right)\right\rangle_{i}-\frac{\left|{ }_{i}\left\langle\operatorname{sign}\left(h_{i}\right)|V|-\operatorname{sign}\left(h_{i}\right)\right\rangle_{i}\right|^{2}}{2\left|h_{i}\right|} .
$$

Escrevendo o operador $\sigma_{i}^{x}$ em função dos operadores de levantamento e abaixamento

$$
\sigma_{i}^{x}=S_{i}^{z+}+S_{i}^{z-}
$$

é possível mostrar que para spin $1 / 2$

$$
\sigma_{i}^{x}\left|\operatorname{sign}\left(h_{i}\right)\right\rangle_{i}=\left|-\operatorname{sign}\left(h_{i}\right)\right\rangle_{i},
$$


como qual podemos obter

$$
{ }_{i}\left\langle\operatorname{sign}\left(h_{i}\right)|V| \operatorname{sign}\left(h_{i}\right)\right\rangle_{i}=0
$$

e

$$
\begin{aligned}
\frac{\left.\left.\right|_{i}\left\langle\operatorname{sign}\left(h_{i}\right)|V|-\operatorname{sign}\left(h_{i}\right)\right\rangle_{i}\right|^{2}}{2\left|h_{i}\right|} & =\frac{\left(J_{i-1} \sigma_{i-1}^{x}+J_{i} \sigma_{i+1}^{x}\right)^{2}}{2\left|h_{i}\right|} \\
& =\frac{J_{i-1}^{2}+J_{i}^{2}}{2\left|h_{i}\right|}+\frac{J_{i-1} J_{i}}{\left|h_{i}\right|} \sigma_{i-1}^{x} \sigma_{i+1}^{x} .
\end{aligned}
$$

Substituindo estes resultados na Hamiltoniana (A.6) obtemos

$$
H \approx E_{0}-\frac{J_{i-1} J_{i}}{\left|h_{i}\right|} \sigma_{i-1}^{x} \sigma_{i+1}^{x}-\sum_{\substack{k=1 \\ k \neq i-1, i}}^{N} J_{k} \sigma_{k}^{x} \sigma_{k+1}^{x}-\sum_{\substack{k=1 \\ k \neq i}}^{N} h_{k} \sigma_{k}^{z}
$$

com

$$
E_{0}=-\left|h_{i}\right|-\frac{J_{i-1}^{2}+J_{i}^{2}}{2\left|h_{i}\right|} .
$$

Vemos que esse tratamento reduz um grau de liberdade do sistema dizimando o sítio $i$ e introduzindo uma interação com valor

$$
\tilde{J}=\frac{J_{i-1} J_{i}}{\left|h_{i}\right|}
$$

entre os sítios $i-1$ e $i+1$ conforme (A.2). É interessante notar que neste processo o novo acoplamento $\tilde{J}$ é menor que o acoplamento $J_{i-1, i}$, portanto, ao dizimar o sítio $i$ devido ao campo $h_{i}$, abaixamos o acoplamento efetivo entre os sítios.

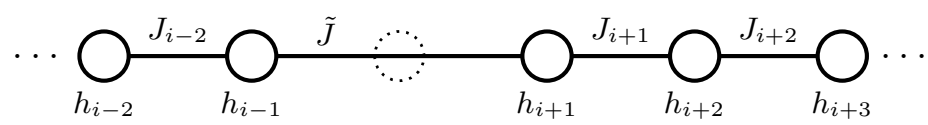

Figura A.2: Cadeia unidimensional após o tratamento perturbativo quando $h_{i}$ é muito maior que os outros termos na vizinhança de $i$.

\section{A.1.2 Dizimação do acoplamento $J_{i}$}

Na subseção anterior tratamos a Hamiltoniana (A.1) perturbativamente quando $h_{i}$ for muito maior que os acoplamentos e campos na vizinhança do sítio $i$. Agora analisaremos 
o caso em que $J_{i}$ é o parâmetro relevante, ou seja, trataremos

$$
H_{0}=-J_{i} \sigma_{i}^{x} \sigma_{i+1}^{x}
$$

exatamente, enquanto que

$$
V=-h_{i} \sigma_{i}^{z}-h_{i+1} \sigma_{i+1}^{z}
$$

será a perturbação.

Usando os autovetores e autovalores na base $\sigma^{x}$

$$
\lambda^{\uparrow}=1 \quad \text { e } \quad|\uparrow\rangle=\frac{1}{\sqrt{2}}\left(\begin{array}{l}
1 \\
1
\end{array}\right),
$$

$\mathrm{e}$

$$
\lambda^{\downarrow}=-1 \quad \text { e } \quad|\downarrow\rangle=\frac{1}{\sqrt{2}}\left(\begin{array}{c}
1 \\
-1
\end{array}\right),
$$

observamos que $H_{0}$ tem dois autovetores de spins paralelos com autovalor $-J_{i}$

$$
|a\rangle=\frac{1}{\sqrt{2}}(|\uparrow \uparrow\rangle+|\downarrow \downarrow\rangle)
$$

$$
|-a\rangle=\frac{1}{\sqrt{2}}(|\uparrow \uparrow\rangle-|\downarrow \downarrow\rangle),
$$

mais dois outros autovetores de spins antiparalelos com autovalor $J_{i}$

$$
|b\rangle=\frac{1}{\sqrt{2}}(|\uparrow \downarrow\rangle+|\downarrow \uparrow\rangle)
$$

e

$$
|-b\rangle=\frac{1}{\sqrt{2}}(|\uparrow \downarrow\rangle-|\downarrow \uparrow\rangle) .
$$

Quando $J_{i}$ for o termo de maior relevância na Hamiltoniana local, $H_{0}-V$, o estado fundamental será definido pelo sinal de $J_{i}$. Caso $J_{i}>0$, o estado fundamental serão aqueles com spins alinhados dados por $|a\rangle$ e $|-a\rangle$, ao passo que $J_{i}<0$ favorecerá spins antiparalelos $|b\rangle,|-b\rangle$.

\section{A.1.2.1 $J_{i}>0$}

Usando as relações presentes na tabela A.1 podemos verificar que a primeira correção na energia é nula (mesmo considerando a degenerescência), enquanto que em segunda 
Tabela A.1: Operadores atuando nos autovetores de $H_{0}$

\begin{tabular}{rlll}
\hline$\sigma_{i, i+1}^{x}$ & $| \pm a\rangle$ & $=$ & $|\mp a\rangle$ \\
$\sigma_{i}^{z}$ & $| \pm a\rangle$ & $= \pm$ & $| \pm b\rangle$ \\
$\sigma_{i+1}^{z}$ & $| \pm a\rangle$ & $=$ & $| \pm b\rangle$ \\
\hline$\sigma_{i}^{x}$ & $| \pm b\rangle$ & $=$ & $|\mp b\rangle$ \\
$\sigma_{i+1}^{x}$ & $| \pm b\rangle$ & $=-$ & $|\mp b\rangle$ \\
$\sigma_{i}^{z}$ & $| \pm b\rangle$ & $= \pm$ & $| \pm a\rangle$ \\
$\sigma_{i+1}^{z}$ & $| \pm b\rangle$ & $=$ & $| \pm a\rangle$ \\
\hline
\end{tabular}

Tabela A.2: Operadores atuando nos autovetores de $H_{0}$

\begin{aligned} & \hline$\langle a|V| b\rangle=h_{i}+h_{i+1} \\ &\langle a|V|-b\rangle=0 \\ &\langle-a|V| b\rangle=0 \\ &\langle-a|V|-b\rangle=-h_{i}+h_{i+1} \\ &$\hline\end{aligned}

ordem e de acordo com a tabela A.2

$$
E^{(2)}=-\frac{h_{i}^{2}+h_{i+1}^{2}}{2\left|J_{i}\right|}-\frac{h_{i} h_{i+1}}{\left|J_{i}\right|}
$$

para o estado $|a\rangle$. Para o estado $|-a\rangle$

$$
E^{(2)}=-\frac{h_{i}^{2}+h_{i+1}^{2}}{2\left|J_{i}\right|}+\frac{h_{i} h_{i+1}}{\left|J_{i}\right|} .
$$

Como o estado $|a\rangle$ e $|-a\rangle$ correspondem a dois spins alinhados nas direções $\hat{z}$ e $-\hat{z}$, podemos interpretar as correções de segunda ordem acima como uma Hamiltoniana

$$
H^{\prime}=-\frac{h_{i}^{2}+h_{i+1}^{2}}{2\left|J_{i}\right|}-\tilde{h} \tilde{\sigma}^{z}
$$

atuando num spin efetivo de "momento magnético 1 e spin $1 / 2$ "com campo

$$
\tilde{h}=\frac{J_{i} J_{i+1}}{\left|h_{i}\right|}
$$

conforme a figura (A.3). É importante notar que o operador $\tilde{\sigma}^{z}$ não é igual a $\sigma^{z}$, contudo a diferença é pequena se $J_{i}$ for suficientemente grande. 


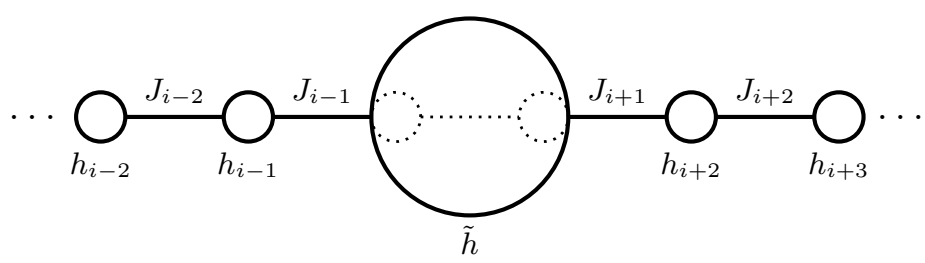

Figura A.3: Renormalização do campo $J_{i}$.

Sendo assim, esse tratamento perturbativo nos leva a Hamiltoniana

$$
H \approx-\left|J_{i}\right|-\frac{h_{i}^{2}+h_{i+1}^{2}}{2\left|J_{i}\right|}-\tilde{h} \tilde{\sigma}^{z}-\sum_{\substack{k=1 \\ k \neq i}}^{N} J_{k} \sigma_{k}^{x} \sigma_{k+1}^{x}-\sum_{\substack{k=1 \\ k \neq i, i+1}}^{N} h_{k} \sigma_{k}^{z}
$$

eliminando um grau de liberdade do sistema.

\section{A.1.2.2 Caso $J_{i}<0$}

Quando $J_{i}<0$ os estados de mais baixa energia são combinações de spins antiparalelos $|b\rangle$ e $|-b\rangle$. A partir da tabela A.2, obtemos as mesmas correções encontradas nas equações (A.26) e (A.27) e, como os estados $|b\rangle$ e $|-b\rangle$ também correspondem a estados de spins paralelos e alinhados com o eixo $z$, podemos concluir que se $J_{i}<0$ a aproximação dada por (A.30) também é válida.

Assim como no caso da dizimação do campo magnético, o tratamento perturbativo do maior acoplamento diminui a escala de energia introduzindo um campo mais fraco $\tilde{h}$. Além do mais, podemos ver a dualidade nas relações (A.17) e (A.29), já que estas equações são idênticas pela troca de $h$ por $J$.

\section{A.1.3 Equações de fluxo}

Nas seções anteriores consideramos o caso em que o acoplamento $J_{i}$ ou o campo $h_{i}$ são muito mais importantes que as outras interações. Em ambos os casos a teoria de perturbação até a segunda ordem elimina um grau de liberdade da Hamiltoniana introduzindo novos campos ou acoplamentos. Este procedimento introduz campos e acoplamentos mais fracos que os anteriores, aproximando o sistema do estado fundamental a temperatura nula. A ideia do algoritmo é fazer sucessivas eliminações dos campos e acoplamentos mais fortes a fim de reduzir o número de graus de liberdade do sistema.

O algoritmo consiste em 
1. Escolher $\Omega=\max \left\{\left|J_{i}\right|,\left|h_{i}\right|\right\}$

2. - Caso $\Omega=\left|J_{i}\right|$, os spins estão sempre alinhados. Dizimamos o acoplamento $J_{i}$ trocando os spins nos sítios $i$ e $i+1$ por um outro spin de momento magnético $\mu_{i}+\mu_{i+1}$ com campo $\tilde{h}=h_{i} h_{i+1} / J_{i}$.

- Caso $\Omega=\left|h_{i}\right|$, o spin sempre estará alinhado com o campo. Dizimamos o spin $i$ e adicionamos um acoplamento entre os sítios $i-1$ e $i+$ com valor $\tilde{J}=J_{i-1} J_{i} / h_{i}$.

Dada a escala de energia $\Omega$, a cada iteração as distribuições de $h$ e $J, P(h, \Omega)$ e $R(J, \Omega)$, têm a escala de energia reduzida em $d \Omega$. Dizimando a maior escala de energia, podemos eliminar $h_{i}=\Omega$ com probabilidade $d \Omega P(\Omega, \Omega)$, ganhando um acoplamento $J_{i} J_{i+1} / \Omega$ ao custo de outros dois acoplamentos $J_{i}$ e $J_{i+1}$. Por outro lado, se $J_{i}=$ $\Omega$, selecionamos o maior acoplamento com probabilidade $d \Omega R(\Omega, \Omega)$ e dizimamos $J_{i}$, perdendo dois campos $h_{i}$ e $h_{i+1}$ e ganhando outro de valor $h_{i} h_{i+1} / \Omega$. Desde de que $P$ seja uma função par, podemos supor $h, J>0$ sem perda de generalidade, obtendo

$$
\begin{aligned}
P(h, \Omega-d \Omega)= & P(h, \Omega)-d \Omega P(\Omega, \Omega) \delta(h-\Omega)+ \\
& +d \Omega R(\Omega, \Omega)\left[-\int_{0}^{\Omega} d h_{i} P\left(h_{i}, \Omega\right) \delta\left(h-h_{i}\right)-\right. \\
& -\int_{0}^{\Omega} d h_{i+1} P\left(h_{i+1}, \Omega\right) \delta\left(h-h_{i+1}\right)+ \\
& \left.\left.+\int_{0}^{\Omega} d h_{i} d h_{i+1} P\left(h_{i}, \Omega\right) P\left(h_{i+1}, \Omega\right) \delta\left(h-\frac{h_{i} h_{i+1}}{\Omega}\right)\right]\right\} \\
& \times[1-d \Omega[P(\Omega, \Omega)+R(\Omega, \Omega)]]^{-1},
\end{aligned}
$$

sendo que o fator $1-d \Omega[P(\Omega, \Omega)+R(\Omega, \Omega)]$ garante a normalização, pois a cada iteração perdemos um número de spins igual a $d \Omega[P(\Omega, \Omega)+R(\Omega, \Omega)]$. Simplificando a equação acima, obtemos quando $h<\Omega$

$$
\begin{aligned}
P(h, \Omega-d \Omega)= & \left\{P(h, \Omega)+d \Omega R(\Omega, \Omega)\left[\int_{h}^{\Omega} d h_{i} \frac{\Omega}{h_{i}} P\left(h_{i}, \Omega\right) P\left(\frac{\Omega h}{h_{i}}, \Omega\right)-2 P(h, \Omega)\right]\right\} \\
& \times[1-d \Omega[P(\Omega, \Omega)+R(\Omega, \Omega)]]^{-1},
\end{aligned}
$$

onde os limites de integração mudam pois a renormalização reduz a energia implicando $h<\min \left\{h_{i}, h_{i+1}\right\}$. Expandindo o lado esquerdo em série de potências em $-d \Omega$, temos 
em primeira ordem

$$
\frac{d P(h, \Omega)}{d \Omega}=P(h, \Omega)[R(\Omega, \Omega)-P(\Omega, \Omega)]-R(\Omega, \Omega) \int_{h}^{\Omega} d h_{i} \frac{\Omega}{h_{i}} P\left(h_{i}, \Omega\right) P\left(\frac{\Omega h}{h_{i}}, \Omega\right) .
$$

A equação para $R(J, \Omega)$ é mesma equação acima pela troca $P \leftrightarrow R$ e $h \leftrightarrow J$ já que as regras de renormalização são simétricas

$$
\frac{d R(J, \Omega)}{d \Omega}=R(J, \Omega)[P(\Omega, \Omega)-R(\Omega, \Omega)]-P(\Omega, \Omega) \int_{J}^{\Omega} d J_{i} \frac{\Omega}{J_{i}} R\left(J_{i}, \Omega\right) R\left(\frac{\Omega J}{J_{i}}, \Omega\right) .
$$

Agora buscaremos por soluções na forma

$$
P(h, \Omega)=\frac{p_{0}(\Omega)}{\Omega}\left(\frac{\Omega}{h}\right)^{1-p_{0}(\Omega)}
$$

$\mathrm{e}$

$$
R(J, \Omega)=\frac{r_{0}(\Omega)}{\Omega}\left(\frac{\Omega}{J}\right)^{1-r_{0}(\Omega)} .
$$

Substituindo estes resultados na equação diferencial de $R$

$$
\begin{gathered}
-\frac{r_{0}^{2}}{\Omega^{2}}+\left(\frac{1}{\Omega}-\frac{r_{0}}{\Omega} \ln \left(\frac{\Omega}{J}\right)\right) \frac{d r_{0}}{d \Omega}=\frac{r_{0}}{\Omega}\left[\frac{p_{0}-r_{0}}{\Omega}\right]-\frac{p_{0} r_{0}^{2}}{\Omega^{2}} \ln \left(\frac{\Omega}{J}\right) \\
\left(\frac{1}{\Omega}-\frac{r_{0}}{\Omega} \ln \left(\frac{\Omega}{J}\right)\right) \frac{d r_{0}}{d \Omega}=\frac{r_{0} p_{0}}{\Omega}\left(\frac{1}{\Omega}-\frac{r_{0}}{\Omega} \ln \left(\frac{\Omega}{J}\right)\right),
\end{gathered}
$$

$\log 0$

$$
\left[p_{0} r_{0}-\Omega \frac{d r_{0}}{d \Omega}\right]\left[\frac{1}{\Omega}-\frac{r_{0}}{\Omega} \ln \left(\frac{\Omega}{J}\right)\right]=0,
$$

portanto obtemos a equação diferencial

$$
\frac{d p_{0}}{d \Gamma}=-p_{0} r_{0}
$$

em termos de

$$
\Gamma=-\ln \Omega
$$

Invocando novamente a simetria da renormalização, é imediato obter a equação para $r_{0}$ pela substituição $r_{0} \leftrightarrow p_{0}$

$$
\frac{d r_{0}}{d \Gamma}=-p_{0} r_{0}
$$


Subtraindo (A.38) de (A.40), notamos que

$$
p_{0}-r_{0}=\text { constante }=2 \Delta,
$$

tornando conveniente introduzir a variável

$$
y_{0}=p_{0}-\Delta=r_{0}+\Delta,
$$

que nos leva a uma única equação diferencial

$$
\frac{d y_{0}}{d \Gamma}=\Delta^{2}-y_{0}^{2}
$$

Estamos particularmente interessados na solução próximo ao ponto crítico, ou seja, quando as distribuição de $h$ e $J$ são simétricas e portanto quando $\Delta \rightarrow 0$. Neste caso a solução é dada por

$$
y_{0}=\frac{1}{\ln \left(\Omega_{0} / \Omega\right)}=\frac{1}{\Gamma-\Gamma_{0}} .
$$

A fim de testar a validade desta solução no ponto fixo do sistema, consideremos a probabilidade de encontrar um acoplamento $J$ no intervalo $(\alpha \Omega, \Omega)$

$$
\int_{\alpha \Omega}^{\Omega} R(J, \Omega) d J=\int_{\alpha \Omega}^{\Omega} \frac{r_{0}}{\Omega}\left(\frac{\Omega}{J}\right)^{1-r_{0}} d J=1-\alpha^{r_{0}}
$$

porém no ponto fixo $y_{0}=r_{0}=1 / \ln \left(\Omega_{0} / \Omega\right) \rightarrow 0$, pois $\Omega \rightarrow 0$, e $1-\alpha^{r_{0}} \rightarrow 0$. Sendo assim todos os acoplamentos são dizimados e a solução proposta deve ser a solução no ponto fixo. Além disso, a distribuição de probabilidades próxima ao ponto fixo

$$
P(h, \Omega)=\frac{1}{h\left(\Gamma-\Gamma_{0}\right)} e^{(\Gamma+\ln h) /\left(\Gamma-\Gamma_{0}\right)} \approx \frac{1}{h \Gamma} e^{(\Gamma+\ln h) / \Gamma} \approx \frac{1}{h \Gamma}
$$

torna-se extremamente larga a medida que $\Gamma \rightarrow \infty(\Omega \rightarrow 0)$, fazendo com que o tratamento perturbativo desse grupo de renormalização fique cada vez melhor.

Para obter uma intuição a respeito do parâmetro $\Delta$, vamos calcular

$$
\begin{aligned}
\frac{d}{d \Omega}\left\langle\left(\frac{J}{h}\right)^{\nu}\right\rangle & =\frac{d}{d \Omega} \int_{0}^{\Omega} d h d J R(J, \Omega) P(h, \Omega)\left(\frac{J}{h}\right)^{\nu} \\
& =P(\Omega, \Omega) \Omega^{-\nu}<J^{\nu}>+R(\Omega, \Omega) \Omega^{\nu}<h^{-\nu}>+ \\
& +\underbrace{\int_{0}^{\Omega} d h d J \frac{d R(J, \Omega)}{d \Omega} P(h, \Omega)\left(\frac{J}{h}\right)^{\nu}}_{A}+\underbrace{\int_{0}^{\Omega} d h d J \frac{d P(h, \Omega)}{d \Omega} R(J, \Omega)\left(\frac{J}{h}\right)^{\nu}}_{B}
\end{aligned}
$$


Usando a equação (A.152) podemos calcular $A$

$$
\begin{aligned}
A & =\left\langle\left(\frac{J}{h}\right)^{\nu}\right\rangle[P(\Omega, \Omega)-R(\Omega, \Omega)]- \\
& -P(\Omega, \Omega) \int_{0}^{\Omega} d J d h\left(\frac{J}{h}\right)^{\nu} P(h, \Omega) \int_{J}^{\Omega} d J_{i} \frac{\Omega}{J_{i}} R\left(J_{i}, \Omega\right) R\left(\frac{\Omega J}{J_{i}}, \Omega\right) .
\end{aligned}
$$

Invertendo a integral em $J$ e $J_{i}$

$$
\begin{aligned}
& A=\left\langle\left(\frac{J}{h}\right)^{\nu}\right\rangle[P(\Omega, \Omega)-R(\Omega, \Omega)]- \\
&-P(\Omega, \Omega) \int_{0}^{\Omega} d J_{i} d h h^{-\nu} P(h, \Omega) \frac{\Omega}{J_{i}} R\left(J_{i}, \Omega\right) \int_{0}^{J_{i}} \frac{\Omega}{J_{i}} d J J^{\nu} R\left(\frac{\Omega J}{J_{i}}, \Omega\right) \\
& A=\left\langle\left(\frac{J}{h}\right)^{\nu}\right\rangle[P(\Omega, \Omega)-R(\Omega, \Omega)]-P(\Omega, \Omega)<J^{\nu}>\Omega^{-\nu}\left\langle\left(\frac{J}{h}\right)^{\nu}\right\rangle .
\end{aligned}
$$

Fazendo cálculos análogos para $B$ obtemos

$$
B=\left\langle\left(\frac{J}{h}\right)^{\nu}\right\rangle[R(\Omega, \Omega)-P(\Omega, \Omega)]-R(\Omega, \Omega)<h^{-\nu}>\Omega^{\nu}\left\langle\left(\frac{J}{h}\right)^{\nu}\right\rangle,
$$

de onde concluímos que

$$
\frac{d}{d \Omega}\left\langle\left(\frac{J}{h}\right)^{\nu}\right\rangle=\left\{1-\left\langle\left(\frac{J}{h}\right)^{\nu}\right\rangle\right\}\left[P(\Omega, \Omega)<J^{\nu}>\Omega^{-\nu}+R(\Omega, \Omega)<h^{-\nu}>\Omega^{\nu}\right] .
$$

É interessante notar que, dado $\nu=\tilde{\nu}$, tal que

$$
\left\langle\left(\frac{J}{h}\right)^{\tilde{\nu}}\right\rangle=1,
$$

$\tilde{\nu}$ permanece invariante durante as iterações do grupo de renormalização. No ponto fixo e para o caso particular em que $\nu=2 \Delta$, obtemos

$$
\left\langle\left(\frac{J}{h}\right)^{2 \Delta}\right\rangle=1 .
$$

Portanto $\tilde{\nu}=2 \Delta$.

Quando $\Delta$ for muito pequeno, podemos fazer uma expansão até a segundar ordem para obter

$$
\Delta \sim<\ln h>-<\ln J>
$$


De acordo com a dualidade do modelo de Ising que nos permite trocar $h$ por $J$, o ponto crítico deve ser simétrico em $h$ e $J$, logo $\Delta$ pode ser usado como o parâmetro de controle ou distância ao ponto crítico. Além do mais, (A.52) nos mostra como quantificar $\Delta$ próximo ao ponto crítico.

\section{A.1.4 Renormalização dos comprimentos}

Na seção anterior consideramos como a distribuição de $h$ e $J$ mudam a cada iteração de renormalização, nesta seção associaremos a cada acoplamento e spin comprimentos $l^{b}$ e $l^{s}$ cuja renormalização nos levará ao comportamento crítico do comprimento de correlação.

Conforme ilustrado na figura (A.2), na renormalização de um campo $h_{i}$ dizimamos um spin e adicionamos um acoplamento mais fraco entre os spins $i-1$ e $i+1$. Dessa forma o novo acoplamento tem comprimento

$$
\tilde{l}^{b}=l_{i-1}^{b}+l_{i}^{s}+l_{i}^{b}
$$

De maneira análoga, quando renormalizamos o acoplamento $J_{i}$, formamos um spin maior cujo comprimento será

$$
\tilde{l}^{s}=l_{i}^{s}+l_{i}^{b}+l_{i+1}^{s}
$$

Com estas regras para a renormalização dos comprimentos, vamos generalizar a equação (A.32) para incluir os valores de $\tilde{l}^{s}$ e $\tilde{l}^{b}$. Começamos considerando distribuições de probabilidade $P(h, l, \Omega)$ e $R(J, l, \Omega)$, relacionadas com as distribuições anteriores através das equações

$$
P(h, \Omega)=\int_{0}^{\infty} d l P(h, l, \Omega) \quad \text { e } \quad R(J, \Omega)=\int_{0}^{\infty} d l R(J, l, \Omega) .
$$

Uma dizimação de acoplamento ocorre com probabilidade $d \Omega R\left(\Omega, l^{b}, \Omega\right)$ e neste processo ganhamos campos $h_{i} h_{i+1} / \Omega$ e comprimentos $l_{i}^{s}+l_{i}^{b}+l_{i+1}^{s}$ ao custo de outros dois campos 
e comprimentos quaisquer. Portanto

$$
\begin{aligned}
P(h, l, \Omega-d \Omega)= & P(h, l, \Omega)-d \Omega P(\Omega, l, \Omega) \delta(h-\Omega) \\
+ & d \Omega \int_{0}^{\infty} d l^{b} d l_{i}^{s} d l_{i+1}^{s} \int_{0}^{\Omega} d h_{i} d h_{i+1} R\left(\Omega, l^{b}, \Omega\right) P\left(h_{i}, l_{i}^{s}, \Omega\right) \times \\
\times & P\left(h_{i+1}, l_{i+1}^{s}, \Omega\right) \delta\left(h-\frac{h_{i} h_{i+1}}{\Omega}\right) \delta\left(l-l^{b}-l_{i}^{s}-l_{i+1}^{s}\right)- \\
& -d \Omega \int_{0}^{\infty} d l^{b} d l_{i} \int_{0}^{\Omega} d h_{i} R\left(\Omega, l^{b}, \Omega\right) P\left(h_{i}, l_{i}^{s}, \Omega\right) \delta\left(h-h_{i}\right) \delta\left(l-l_{i}\right)- \\
- & \left.d \Omega \int_{0}^{\infty} d l^{b} d l_{i+1} \int_{0}^{\Omega} d h_{i+1} R\left(\Omega, l^{b}, \Omega\right) P\left(h_{i}, l_{i+1}^{s}, \Omega\right) \delta\left(h-h_{i+1}\right) \delta\left(l-l_{i+1}\right)\right\} / \\
/[ & -d \Omega[P(\Omega, \Omega)+R(\Omega, \Omega)]] . \\
P(h, l, \Omega- & d \Omega)=\{P(h, l, \Omega)-d \Omega P(\Omega, l, \Omega) \delta(h-\Omega)-2 d \Omega R(\Omega, \Omega) P(h, l, \Omega)+ \\
& +d \Omega \int_{0}^{l} d l_{i}^{s} \int_{0}^{l-l_{i}^{s}} d l_{i+1}^{s} \int_{h}^{\Omega} d h_{i} \frac{\Omega}{h_{i}} \times \\
& \left.\times R\left(\Omega, l-l_{i}^{s}-l_{i+1}^{s}, \Omega\right) P\left(h_{i}, l_{i}^{s}, \Omega\right) P\left(\frac{\Omega h}{h_{i}}, l_{i+1}^{s}, \Omega\right)\right\} / \\
& /[1-d \Omega[P(\Omega, \Omega)+R(\Omega, \Omega)]] .
\end{aligned}
$$

Realizando a expansão em $-d \Omega$ obtemos a generalização da equação (A.32) quando $h<\Omega$

$$
\begin{aligned}
\frac{d}{d \Omega} P(h, l, \Omega) & =P(h, l, \Omega)[R(\Omega, \Omega)-P(\Omega, \Omega)]- \\
& -\int_{0}^{l} d l_{i}^{s} \int_{0}^{l-l_{i}^{s}} d l_{i+1}^{s} \int_{h}^{\Omega} d h_{i} \frac{\Omega}{h_{i}} \times \\
& \times R\left(\Omega, l-l_{i}^{s}-l_{i+1}^{s}, \Omega\right) P\left(h_{i}, l_{i}^{s}, \Omega\right) P\left(\frac{\Omega h}{h_{i}}, l_{i+1}^{s}, \Omega\right) .
\end{aligned}
$$

Para simplificar essa equação fazemos a mudança de variável

$$
l^{\prime}=l-l_{i+1}^{s}
$$

e a transformada de Laplace

$$
\tilde{P}(h, \lambda, \Omega)=\int_{0}^{\infty} d l e^{-l \lambda} P(h, l, \Omega), \quad \tilde{R}(J, \lambda, \Omega)=\int_{0}^{\infty} d l e^{-l \lambda} R(J, l, \Omega),
$$


obtendo

$$
\begin{aligned}
\frac{d}{d \Omega} \tilde{P}(h, \lambda, \Omega) & =\tilde{P}(h, \lambda, \Omega)[R(\Omega, \Omega)-P(\Omega, \Omega)]- \\
& -\tilde{R}(\Omega, \lambda, \Omega) \int_{h}^{\Omega} d h_{i} \frac{\Omega}{h_{i}} \tilde{P}\left(h_{i}, \lambda, \Omega\right) \tilde{P}\left(\frac{\Omega h}{h_{i}}, \lambda, \Omega\right)
\end{aligned}
$$

$\mathrm{e}$

$$
\begin{aligned}
\frac{d}{d \Omega} \tilde{R}(J, \lambda, \Omega) & =\tilde{R}(J, \lambda, \Omega)[P(\Omega, \Omega)-R(\Omega, \Omega)]- \\
& -\tilde{P}(\Omega, \lambda, \Omega) \int_{J}^{\Omega} d J_{i} \frac{\Omega}{J_{i}} \tilde{R}\left(J_{i}, \lambda, \Omega\right) \tilde{R}\left(\frac{\Omega J}{J_{i}}, \lambda, \Omega\right)
\end{aligned}
$$

pela permutação $P \leftrightarrow R, J \leftrightarrow h$.

Para resolver essas equações usamos soluções parecidas com as equações (A.35) e (A.36)

$$
\tilde{P}=\frac{\pi(\lambda, \Omega)}{\Omega}\left(\frac{\Omega}{h}\right)^{1-p_{l}(\lambda, \Omega)}
$$

$\mathrm{e}$

$$
\tilde{R}=\frac{\rho(\lambda, \Omega)}{\Omega}\left(\frac{\Omega}{J}\right)^{1-r_{l}(\lambda, \Omega)},
$$

relacionadas com $P$ e $R$ através de $\pi(0, \Omega)=p_{l}(0, \Omega)=p_{0}(\Omega)$ e $\rho(0, \Omega)=r_{l}(0, \Omega)=$ $r_{0}(\Omega)$. Substituindo na equação (A.59) obtemos

$$
\frac{\pi}{\Omega} \ln \left(\frac{\Omega}{h}\right)\left\{\frac{d p_{l}}{d \Omega}-\frac{\rho \pi}{\Omega}\right\}+\left\{\frac{1}{\Omega} \frac{d \pi}{d \Omega}-\frac{\pi}{\Omega^{2}}\left[p_{l}-p_{0}+r_{0}\right]\right\}=0,
$$

que pode ser escrito em termos de $\Gamma=-\ln \Omega$

$$
\pi \ln \left(\frac{\Omega}{h}\right)\left\{\frac{d p_{l}}{d \Gamma}+\rho \pi\right\}-\left\{\frac{d \pi}{d \Gamma}+\pi\left[p_{l}-p_{0}+r_{0}\right]\right\}=0
$$

e satisfeita somente se

$$
\frac{d p_{l}}{d \Gamma}=-\pi \rho \quad \text { e } \quad \frac{d \pi}{d \Gamma}=-\pi\left[p_{l}-p_{0}+r_{0}\right]^{1} .
$$

Devido à simetria do modelo, as equações diferenciais para $r_{l}$ e $\rho$ podem ser escritas

\footnotetext{
${ }^{1}$ Esta equação é diferente da encontrada em [94]
} 
101 A.1. Solução do modelo de Ising aleatório no campo transverso unidimensional

pelas permutações $p_{l} \leftrightarrow r_{l}$ e $\pi \leftrightarrow \rho$

$$
\frac{d r_{l}}{d \Gamma}=-\pi \rho \quad \text { e } \quad \frac{d \rho}{d \Gamma}=-\rho\left[r_{l}-r_{0}+p_{0}\right]^{1} .
$$

Para resolver as equações (A.64) e (A.65) devemos notar que

$$
\frac{d}{d \Gamma}\left(p_{l}-r_{l}\right)=0 \Rightarrow p_{l}-r_{l}=\text { constante }=2 \epsilon(\lambda)
$$

e

$$
\frac{d}{d \Gamma}\left(p_{l} r_{l}-\rho \pi\right)=0 \Rightarrow p_{l} r_{l}-\rho \pi=\text { constante }=\zeta^{2},
$$

que implicam

$$
\frac{d}{d \Gamma}(\ln \pi-\ln \rho)=2(2 \Delta-\epsilon) \Rightarrow \pi=A(\lambda) \rho e^{2(2 \Delta-\epsilon)\left(\Gamma-\Gamma_{0}\right)} .
$$

Se $\lambda=0$, isto é, quando $\rho=r_{0}$ e $\pi=p_{0}$ obtemos $A=p_{0} e^{-2 \Delta\left(\Gamma-\Gamma_{0}\right)} / r_{0}$. Sendo assim definindo

$$
y_{l}=p_{l}+\epsilon(\lambda)=r_{l}-\epsilon(\lambda)
$$

as equações diferenciais para $p_{l}$ e $r_{l}$ são resumidas a

$$
\frac{d y_{l}}{d \Gamma}=-A \rho^{2} e^{2(2 \Delta-\epsilon) \Gamma},
$$

cujo segundo membro pode ser escrito apenas em termos de $y_{l}$ através da conservação (A.67)

$$
y_{l}^{2}-A \rho^{2} e^{2(2 \Delta-\epsilon)}=\zeta^{2}+\epsilon^{2}=\theta^{2}
$$

na forma

$$
\frac{d y_{l}}{d \Gamma}=\left(\theta-y_{l}\right)\left(\theta+y_{l}\right)
$$

cuja solução é

$$
y_{l}=\frac{\theta \tilde{y}_{0}(\lambda)+\theta^{2} \tanh \left[\theta\left(\Gamma-\Gamma_{0}\right)\right]}{\theta+\tilde{y}_{0}(\lambda) \tanh \left[\theta\left(\Gamma-\Gamma_{0}\right)\right]} .
$$

Para finalmente calcular comprimento médio dos clusters de spins, devemos notar que, através da transformada de Laplace (A.58), o comprimento médio dos clusters de spins pode ser calculado através de

$$
\bar{l}^{s}=\lim _{\lambda \rightarrow 0} \frac{1}{\lambda}\left[1-\frac{\rho(\lambda, \Omega)}{r_{l}(\lambda, \Omega)}\right] .
$$

\footnotetext{
${ }^{1}$ Idem nota acima.
} 
Perto do ponto crítico e quando $\lambda \rightarrow 0$, manipulando a equação (A.71) podemos obter

$$
1-\frac{\rho(\lambda, \Omega)}{r_{l}(\lambda, \Omega)} \sim \frac{\zeta^{2}(\lambda, \Omega)}{r_{l}^{2}(\lambda, \Omega)},
$$

que pode ser inserido na equação (A.74) para calcular o comportamento crítico do comprimento de correlação supondo que

$$
\lim _{\lambda \rightarrow 0} \frac{\zeta^{2}}{\lambda}=\text { constante. }
$$

Se $\lambda=0, r_{l}=r_{0}$, e no ponto crítico,$r_{0}=y_{0}$, portanto através da solução obtida na seção anterior (A.44) encontramos

$$
\bar{l}^{s} \sim\left[\ln \left(\frac{\Omega_{0}}{\Omega}\right)\right]^{2}
$$

obtendo o expoente crítico de tunelamento

$$
\psi=\frac{1}{2} .
$$

Por outro lado, no ponto fixo $y_{0}^{2} \sim \Delta^{2}$ (A.43), logo

$$
\bar{l}^{s} \sim \Delta^{-2}
$$

de onde concluímos que

$$
\nu_{\perp}=2
$$

\section{A.1.5 Renomalização do momento magnético}

Nesta seção indicaremos apenas os principais pontos do cálculo da renormalização do momento magnético, pois é praticamente idêntico ao anterior.

Ao renormalizar um acoplamento trocanos os sítios acoplados por um sítio cujo momento magnético total do cluster de spins é somado (A.3), logo

$$
\tilde{\mu}=\mu_{i}+\mu_{i+1} .
$$

A partir dessa relação obtemos a equação diferencial para a densidade de probabilidade 
103 A.1. Solução do modelo de Ising aleatório no campo transverso unidimensional

se $h<\Omega$

$$
\begin{aligned}
\frac{d P(h, \mu, \Omega)}{d \Omega} & =P(h, \mu, \Omega)[R(\Omega, \Omega)-P(\Omega, \Omega)]- \\
& -R(\Omega, \Omega) \int_{0}^{\mu} d \mu_{i} \int_{h}^{\Omega} d h_{i} \frac{\Omega}{h_{i}} P\left(h_{i}, \mu_{i}, \Omega\right) P\left(\frac{\Omega h}{h_{i}}, \mu-\mu_{i}, \Omega\right),
\end{aligned}
$$

onde aplicamos a transformada de Laplace

$$
\tilde{P}(h, s, \Omega)=\int_{0}^{\infty} d \mu e^{-s \mu} P(h, \mu, \Omega)
$$

a fim de obter uma equação simplificada

$$
\begin{aligned}
\frac{d \tilde{P}(h, s, \Omega)}{d \Omega} & =\tilde{P}(h, s, \Omega)[R(\Omega, \Omega)-P(\Omega, \Omega)]- \\
& -R(\Omega, \Omega) \int_{h}^{\Omega} d h_{i} \frac{\Omega}{h_{i}} \tilde{P}\left(h_{i}, s, \Omega\right) \tilde{P}\left(\frac{\Omega h}{h_{i}}, s, \Omega\right) .
\end{aligned}
$$

Substituindo o ansatz

$$
\tilde{P}(h, s, \Omega)=\frac{\pi_{\mu}(s, \Omega)}{\Omega}\left(\frac{\Omega}{h}\right)^{1-p_{\mu}(s, \Omega)}
$$

obtemos as equações diferenciais

$$
\frac{d p_{\mu}}{d \Gamma}=-\pi_{\mu} r_{0} \quad \text { e } \quad \frac{d \pi_{\mu}}{d \Gamma}=-\pi_{\mu}\left(p_{\mu}+r_{0}-p_{0}\right)
$$

Quando $s$ for pequeno, podemos substituir as expansões

$$
p_{\mu}=p_{0}+s p_{1}(\Omega) \quad \text { e } \quad \pi_{\mu}=p_{0}+s \pi_{1}(\Omega)
$$

obtendo em primeira ordem em $s$

$$
\frac{d p_{1}}{d \Gamma}=-r_{0} \pi_{1} \quad \text { e } \quad \frac{d \pi_{1}}{d \Gamma}=-p_{0} p_{1}-r_{0} \pi_{1}
$$

Dividindo por (A.38) podemos manipular as duas equações para obter

$$
\left(y_{0}^{2}-\Delta^{2}\right) \frac{d^{2} p_{1}}{d y_{0}^{2}}=p_{1}
$$

No ponto crítico, a equação acima é uma equação de Euler simplificada admitindo 
soluções $y_{0}^{(1 \pm \sqrt{5}) / 2}$. Portanto a solução do sistema de equações é dada por

$$
p_{1}=D y_{0}^{(1+\sqrt{5}) / 2}+E y_{0}^{(1+\sqrt{5}) / 2}
$$

$\mathrm{e}$

$$
\pi_{1}=\frac{1}{2}\left((1+\sqrt{5}) D y_{0}^{(1+\sqrt{5}) / 2}+(1-\sqrt{5}) E y_{0}^{(1-\sqrt{5}) / 2}\right) .
$$

Analogamente ao comprimento médio dos spins calculado na seção anterior, o momento magnético é dado por

$$
\bar{\mu}=\lim _{s \rightarrow 0}\left[1-\frac{\pi_{\mu}}{p_{\mu}}\right]=\left.\frac{p_{1}-\pi_{1}}{p_{0}}\right|_{s=0}
$$

sendo que perto do ponto fixo $\Omega \rightarrow 0$, ou seja $y_{0} \rightarrow 0$, o comportamento crítico será dominado pela menor potência em $y_{0}$. Sendo assim

$$
\bar{\mu} \sim y_{0}^{-\frac{(1+\sqrt{5})}{2}} \sim\left[\ln \left(\frac{\Omega_{0}}{\Omega}\right)\right]^{\frac{1+\sqrt{5}}{2}}
$$

com expoente crítico

$$
\phi=\frac{1+\sqrt{5}}{2} .
$$

Para calcular e expoente $\beta$ basta notar que

$$
m=\frac{\bar{\mu}}{\bar{l}},
$$

$\log 0$

$$
m \sim \frac{\Delta^{-(1+\sqrt{5}) / 2}}{\Delta^{-2}} \sim \Delta^{\frac{3-\sqrt{5}}{2}}
$$

e

$$
\beta=\frac{3-\sqrt{5}}{2}
$$

\section{A.2 Comportamento crítico do processo de contato com desordem congelada}

Faremos a solução do comportamento crítico do processo de contato com desordem congelada. A solução será feita através do mapeamento da cadeia de Markov deste modelo em uma "equação de Schrödinger com uma Hamiltoniana de spin 1/2". Devido à semelhança deste mapeamento com o modelo de Ising aleatório no campo transverso, 
usaremos a mesma técnica apresentada na seção anterior para obter a solução. Através dos resultados obtidos, concluiremos que, ao menos para desordem forte o suficiente, o processo de contato de desordem congelada está na mesma classe de universalidade do modelo de Ising aleatório no campo transverso, ambos em uma dimensão.

\section{A.2.1 Equação mestra do processo de contato com desordem conge- lada}

No processo de contato os sítios podem estar infectados ou recuperados. A cada instante um sitio infectado pode infectar um de seus vizinhos com probabilidade $p^{\lambda} / z, z$ é o número de coordenação, ou um infectado recupera-se espontaneamente com probabilidade $p^{\mu}$. Denotando a probabilidade de encontrar o sistema num estado $\sigma$ e no instante $t$ com

$$
\left|P_{t}\left(\sigma_{t}\right)\right\rangle=\left|\sigma_{1}\right\rangle \otimes\left|\sigma_{2}\right\rangle \otimes \ldots\left|\sigma_{N-1}\right\rangle \otimes\left|\sigma_{N}\right\rangle
$$

onde

$$
\sigma_{i}=\left(\begin{array}{c}
a_{i} \\
b_{i}
\end{array}\right) \quad\left(a_{i}+b_{i}=1\right)
$$

se tratarmos

$$
|\uparrow\rangle=\left(\begin{array}{l}
1 \\
0
\end{array}\right)=\text { sítio recuperado }
$$

e

$$
|\downarrow\rangle=\left(\begin{array}{l}
0 \\
1
\end{array}\right)=\text { sítio infectado, }
$$

a evolução deste modelo num estado $\sigma$ com desordem congelada uma dimensão pode ser descrito por

$$
\begin{aligned}
\left|P_{t+\Delta t}\left(\sigma_{t+\Delta t}\right)\right\rangle=\frac{1}{2}\left\{\frac{1}{2(N-1)} \sum_{k=1}^{N-1}\left[\hat{R}_{k} n_{k+1}+\hat{R}_{k+1} n_{k}+\hat{I}_{k} m_{k+1}+\hat{I}_{k+1} m_{k}\right]\right. \\
\left.+\frac{1}{N} \sum_{k=1}^{N} \hat{M}_{k}\right\}\left|P_{t}\left(\sigma_{t}\right)\right\rangle
\end{aligned}
$$

no qual

$$
\hat{R}_{k}=\left(\begin{array}{cc}
1-p_{k}^{\lambda} / 2 & 0 \\
p_{k}^{\lambda} / 2 & 1
\end{array}\right)_{k} \quad \text { e } \quad \hat{M}_{k}=\left(\begin{array}{cc}
1 & p_{k}^{\mu} \\
0 & 1-p_{k}^{\mu}
\end{array}\right)_{k}
$$


são responsáveis pela probabilidade de infecção e recuperação, enquanto que

$$
n_{k}=\left(\begin{array}{ll}
0 & 0 \\
0 & 1
\end{array}\right)_{k} \text { e } m_{k}=\left(\begin{array}{ll}
1 & 0 \\
0 & 0
\end{array}\right)_{k}
$$

verificam a existência ou ausência de um infectado no sítio $k$.

Para passar para a equação a tempo contínuo, notamos que em um intervalo de infinitesimal de tempo $\Delta t$ a probabilidade de mudança de estado é pequena. Assim, através da transformação

$$
\hat{X}=I-\tilde{X} \Delta t
$$

em $\hat{R}_{k}, \hat{I}_{k}$ e $\hat{M}_{k}$, é possível encontrar

$$
\begin{aligned}
\left|P_{t+\Delta t}\left(\sigma_{t+\Delta t}\right)\right\rangle & =\left|P_{t}\left(\sigma_{t}\right)\right\rangle-\frac{\Delta t}{2}\left\{\frac{1}{2(N-1)} \sum_{k=1}^{N-1}\left[\tilde{R}_{k} n_{k+1}+\tilde{R}_{k+1} n_{k}\right]\right. \\
& \left.+\frac{1}{N} \sum_{n=1}^{N} \tilde{M}_{k}\right\}\left|P_{t}\left(\sigma_{t}\right)\right\rangle .
\end{aligned}
$$

Tomando $\Delta t \rightarrow 0$ escrevemos uma equação diferencial

$$
\frac{d}{d t}|P(\sigma)\rangle=-\frac{1}{2}\left\{\frac{1}{2(N-1)} \sum_{k=1}^{N-1}\left[\tilde{R}_{k} n_{k+1}+\tilde{R}_{k+1} n_{k}\right]+\frac{1}{N} \sum_{n=1}^{N} \tilde{M}_{k}\right\}\left|P_{t}\left(\sigma_{t}\right)\right\rangle .
$$

Se o número de partículas for muito grande $(N-1 \approx N)$, podemos renormalizar o tempo $t \rightarrow t / 2 N$

$$
\frac{d}{d t}|P(\sigma)\rangle=-\left\{\frac{1}{2} \sum_{k=1}^{N-1}\left[\tilde{R}_{k} n_{k+1}+\tilde{R}_{k+1} n_{k}\right]+\sum_{n=1}^{N} \tilde{M}_{k}\right\}\left|P_{t}\left(\sigma_{t}\right)\right\rangle
$$

ou ainda,

$$
\frac{d}{d t}|P(\sigma)\rangle=-\left\{\sum_{k=1}^{N-1} \frac{\lambda_{k}}{2}\left[R_{k} n_{k+1}+R_{k+1} n_{k}\right]+\sum_{n=1}^{N} \mu_{k} M_{k}\right\}\left|P_{t}\left(\sigma_{t}\right)\right\rangle,
$$

com

$$
R_{k}=\left(\begin{array}{cc}
1 & 0 \\
-1 & 0
\end{array}\right)_{k}, M_{k}=\left(\begin{array}{cc}
0 & -1 \\
0 & 1
\end{array}\right)_{k}, \lambda_{k}=\lim _{\Delta t \rightarrow 0} \frac{p_{k}^{\lambda}}{2 \Delta t} \quad \text { e } \quad \mu_{k}=\lim _{\Delta t \rightarrow 0} \frac{p_{k}^{\mu}}{\Delta t} .
$$

A equação mestra (A.108) define um "formalismo Hamiltoniano", pois é semelhante 
a uma equação de Schrödinger

$$
\frac{d}{d t}|P(\sigma, t)\rangle=-H|P(\sigma, t)\rangle
$$

sendo

$$
H=\sum_{k=1}^{N-1} \frac{\lambda_{k}}{2}\left[R_{k} n_{k+1}+R_{k+1} n_{k}\right]+\sum_{n=1}^{N} \mu_{k} M_{k}
$$

o gerador desta cadeia de Markov. Apesar desta semelhança, é importante ressaltar que este problema não é quântico. De fato $|P(t)\rangle$ e $H$ representam a probabilidade e o gerador que em geral não é hermitiano.

Deduzida a equação mestra deste problema, seguiremos o mesmo procedimento realizado para o modelo de Ising aleatório no campo transverso. Portanto trataremos o termo $H$ perturbativamente, no qual será possível obter as taxas renormalizadas para depois escrever as equações de fluxo das distribuições de probabilidade de $\lambda$ e $\mu$.

\section{A.2.2 Teoria de perturbação estocástica}

De forma semelhante à teoria de perturbação da mecânica quântica, deduziremos a teoria de perturbação estocástica. Este desenvolvimento difere do quântico pois $H$ não é hermitiano, assim se faz necessário levar em consideração não só os autovetores à direita, mas também os autovetores à esquerda.

Consideremos a equação mestra na forma (A.110) com

$$
H=H_{0}+V
$$

o gerador deste processo. Seguindo a rotina da teoria de perturbação quântica, temos a solução exata de $H_{0}$ e queremos saber como a perturbação $V$ afeta os autovalores e autovetores de $H$. Neste contexto, ter a solução de $H_{0}$ significa ter os autovalores $\Lambda_{i}^{(0)}$ e correspondentes autovetores à esquerda $\left\langle\phi_{i}^{(0)}\right|$ e à direita $\left|\psi_{i}^{(0)}\right\rangle$ que satisfazem a relação de completeza

$$
\sum_{i}\left|\psi_{i}^{(0)}\right\rangle\left\langle\phi_{i}^{(0)}\right|=I
$$

e ortonormalidade

$$
\left\langle\phi_{i}^{(0)} \mid \psi_{i}^{(0)}\right\rangle=\delta_{i j} .
$$

Além disso, é interessante lembrar que o autovetor correspondente ao estado estacionário tem autovalor nulo e que todos os outros autovalores têm parte real positiva. 
Para resolver o problema, escrevemos a equação para o $n$-ésimo autovalor e autovetor

$$
H\left|\Psi_{n}\right\rangle=\left(H_{0}+V\right)\left|\Psi_{n}\right\rangle=\Lambda_{n}\left|\Psi_{n}\right\rangle,
$$

com as expansões

$$
\begin{gathered}
\left|\Psi_{n}\right\rangle=\left|\psi_{n}^{(0)}\right\rangle+\left|\psi_{n}^{(1)}\right\rangle+\left|\psi_{n}^{(2)}\right\rangle+\ldots, \\
\left\langle\Phi_{n}\right|=\left\langle\phi_{n}^{(0)}\right|+\left\langle\phi_{n}^{(1)}\right|+\left\langle\phi_{n}^{(2)}\right|+\ldots,
\end{gathered}
$$

$\mathrm{e}$

$$
\Lambda_{n}=\Lambda_{n}^{(0)}+\Lambda_{n}^{(1)}+\Lambda_{n}^{(2)}+\ldots,
$$

no qual o índice entre parênteses indica a ordem da expansão. Em primeira ordem, obtemos

$$
H_{0}\left|\psi_{n}^{(1)}\right\rangle+V\left|\psi_{n}^{(0)}\right\rangle=\Lambda_{n}^{(0)}\left|\psi_{n}^{(1)}\right\rangle+\Lambda_{n}^{(1)}\left|\psi_{n}^{(0)}\right\rangle
$$

sendo possível isolar $\Lambda_{n}^{(1)}$ tomando o produto com $\left\langle\phi^{(0)}\right|$ à esquerda, e usando respectiva a equação de autovalor $\left\langle\phi_{n}^{(0)}\right| H_{0}=\Lambda^{(0)}\left\langle\phi_{n}^{(0)}\right|$

$$
\Lambda_{n}^{(1)}=\left\langle\phi_{n}^{(0)}|V| \psi_{n}^{(0)}\right\rangle \text {. }
$$

Para obter a primeira correção do autovetor à direita, escrevemos $\left|\psi_{n}^{(1)}\right\rangle$ como uma combinação linear autovetores à direita de ordem zero

$$
\left|\psi_{n}^{(1)}\right\rangle=\sum_{i \neq n} c_{i}\left|\psi_{n}^{(0)}\right\rangle
$$

Inserindo a equação acima em (A.119) e tomando o produto à esquerda com $\left\langle\phi_{i}^{(0)}\right|$, é possível obter os coeficientes $c_{i}$, logo

$$
\left|\psi_{n}^{(1)}\right\rangle=\sum_{i \neq n} \frac{\left\langle\phi_{i}^{(0)}|V| \psi_{n}^{(0)}\right\rangle}{\Lambda_{n}^{(0)}-\Lambda_{i}^{(0)}}\left|\psi_{i}^{(0)}\right\rangle .
$$

Passamos agora para o cálculo da segunda correção do autovalor. A equação em segunda ordem é

$$
H_{0}\left|\psi_{n}^{(2)}\right\rangle+V\left|\psi_{n}^{(1)}\right\rangle=\Lambda_{n}^{(0)}\left|\psi_{n}^{(2)}\right\rangle+\Lambda_{n}^{(1)}\left|\psi_{n}^{(1)}\right\rangle+\Lambda_{n}^{(2)}\left|\psi_{n}^{(0)}\right\rangle .
$$

Substituindo (A.122), tomando o produto à esquerda com $\left\langle\phi_{n}^{(0)}\right|$ e usando a relação de 
ortogonalidade, encontramos

$$
\Lambda_{n}^{(2)}=\sum_{i \neq n} \frac{\left\langle\phi_{n}^{(0)}|V| \psi_{i}^{(0)}\right\rangle\left\langle\phi_{i}^{(0)}|V| \psi_{n}^{(0)}\right\rangle}{\Lambda_{n}^{(0)}-\Lambda_{i}^{(0)}} .
$$

Onde a ordem dos termos foi mantida, pois, como veremos mais adiante, usaremos este cálculo com termos que não comutam.

\section{A.2.2.1 Estados degenerados}

Se temos dois estados degenerados $\left|\psi_{a}^{(0)}\right\rangle$ e $\left|\psi_{b}^{(0)}\right\rangle$, com respectivos autovetores à esquerda $\left\langle\phi_{a}^{(0)}\right|$ e $\left\langle\phi_{b}^{(0)}\right|$, o estado $\left|\psi_{n}^{(0)}\right\rangle$ será uma combinação linear deles

$$
\left|\psi_{n}^{(0)}\right\rangle=\alpha\left|\psi_{a}^{(0)}\right\rangle+\beta\left|\psi_{b}^{(0)}\right\rangle \text {. }
$$

Multiplicando a equação da primeira correção (A.119) à esquerda por $\left\langle\phi_{a}^{(0)}\right|$ e $\left\langle\phi_{b}^{(0)}\right|$, obtemos duas equações

$$
\alpha \Lambda_{n}^{(1)}=\alpha\left\langle\phi_{a}^{(0)}|V| \psi_{a}^{(0)}\right\rangle+\beta\left\langle\phi_{a}^{(0)}|V| \psi_{b}^{(0)}\right\rangle
$$

e

$$
\beta \Lambda_{n}^{(1)}=\alpha\left\langle\phi_{b}^{(0)}|V| \psi_{a}^{(0)}\right\rangle+\beta\left\langle\phi_{b}^{(0)}|V| \psi_{b}^{(0)}\right\rangle .
$$

Supondo $\alpha$ e $\beta \neq 0, \Lambda_{n}^{(1)}$ é a raiz de uma equação quadrática

$$
\Lambda_{n}^{(1)}=\frac{1}{2}\left[W_{a a}+W_{b b} \pm \sqrt{\left(W_{a a}-W_{b b}\right)^{2}+4 W_{a b} W_{b a}}\right],
$$

onde $W_{i j}(i, j \in\{a, b\})$ são os elementos de matriz $\left\langle\phi_{i}^{(0)}|V| \psi_{j}^{(0)}\right\rangle$.

\section{A.2.3 Dizimação da taxa de recuperação}

Passamos a tratar o caso em que o termo mais relevante é

$$
H_{0}=\mu_{k} M_{k}
$$

e a perturbação será dada pelo acoplamento com os sítios vizinhos

$$
V=\frac{\lambda_{k-1}}{2}\left[R_{k-1} n_{k}+R_{k} n_{k-1}\right]+\frac{\lambda_{k}}{2}\left[R_{k} n_{k+1}+R_{k+1} n_{k}\right] .
$$


Neste caso esperamos que $\mu_{k} \gg \lambda_{k, k-1} / 2$ e faremos uma expansão perturbativa quando estamos perto do estado estacionário, ou quando o autovalor mais relevante é o nulo.

Os autovalores e autovetores de $H_{0}$ são

$$
\Lambda_{0}^{(0)}=0, \quad{ }_{k}\left\langle\phi_{0}^{(0)}\right|=\left(\begin{array}{ll}
1 & 1
\end{array}\right)_{k} \quad \text { e }\left|\psi_{0}^{(0)}\right\rangle_{k}=\left(\begin{array}{l}
1 \\
0
\end{array}\right)_{k},
$$

que correspondem ao estado estacionário, e

$$
\Lambda_{1}^{(0)}=\mu_{k}, \quad{ }_{k}\left\langle\phi_{1}^{(0)}\right|=\left(\begin{array}{ll}
0 & 1
\end{array}\right)_{k} \quad \text { e }\left|\psi_{1}^{(0)}\right\rangle_{k}=\left(\begin{array}{c}
-1 \\
1
\end{array}\right)_{k} .
$$

Usando as relações ${ }_{k}\left\langle\phi_{0}^{(0)}\right| R_{k}=0$ e $n_{k}\left|\psi_{0}^{(0)}\right\rangle_{k}=0$, é possível verificar que a primeira correção é nula, isto é, $\Lambda_{0}^{(1)}=0$.

Para obter a correção do autovalor em segunda ordem, $\Lambda_{0}^{(2)}$, calculamos

$$
{ }_{k}\left\langle\phi_{1}^{(0)}|V| \psi_{0}^{(0)}\right\rangle_{k}=-\frac{\lambda_{k-1}}{2} n_{k-1}-\frac{\lambda_{k}}{2} n_{k+1}
$$

e

$$
{ }_{k}\left\langle\phi_{0}^{(0)}|V| \psi_{1}^{(0)}\right\rangle_{k}=\frac{\lambda_{k-1}}{2} R_{k-1}+\frac{\lambda_{k}}{2} R_{k+1} .
$$

Notando que $R_{i} n_{i}=0$, obtemos

$$
\Lambda_{0}^{(2)}=\frac{1}{2} \frac{\lambda_{k-1} \lambda_{k}}{2 \mu_{k}}\left[R_{k-1} n_{k+1}+R_{k+1} n_{k-1}\right] .
$$

Então o operador de evolução pode ser aproximado por

$$
\begin{aligned}
H & \approx \Lambda_{0}^{(1)}+\Lambda_{0}^{(2)}+\sum_{\substack{i=1 \\
i \neq k, k-1}}^{N-1} \frac{\lambda_{i}}{2}\left[R_{i} n_{i+1}+R_{i+1} n_{i}\right]+\sum_{\substack{n=1 \\
i \neq k}}^{N} \mu_{i} M_{i} \\
& \approx \frac{1}{2} \frac{\lambda_{k-1} \lambda_{k}}{2 \mu_{k}}\left[R_{k-1} n_{k+1}+R_{k+1} n_{k-1}\right]+\sum_{\substack{i=1 \\
i \neq k, k-1}}^{N-1} \frac{\lambda_{i}}{2}\left[R_{i} n_{i+1}+R_{i+1} n_{i}\right]+\sum_{\substack{n=1 \\
i \neq k}}^{N} \mu_{i} M_{i}
\end{aligned}
$$

De acordo com a equação acima, a interpretação da dizimação de uma taxa de recuperação é a mesma da dizimação de um campo $h$, ou seja, o tratamento perturbativo 
remove um grau de liberdade do sistema e introduz um acoplamento

$$
\tilde{\lambda}=\frac{\lambda_{k} \lambda_{k-1}}{2 \mu_{k}}
$$

Entretanto, diferente do do campo $\tilde{h}$ na equação (A.29), temos um fator $1 / 2$. Este fator pode ser justificado devido à condição $\mu_{k} \gg \lambda_{k, k-1} / 2$, que implica que a variável relevante não é $\mu_{k}$ mas $2 \mu_{k}$, portanto a equação de $\tilde{h}$ é equivalente à $\tilde{\lambda}$.

\section{A.2.4 Dizimação de um acoplamento}

Passamos a analisar o caso em que o acoplamento, com Hamiltoniana

$$
H_{0}=\frac{\lambda_{k}}{2}\left[R_{k} n_{k+1}+R_{k+1} n_{k}\right]
$$

é o termo relevante, ao passo que

$$
V=\mu_{k} M_{k}+\mu_{k+1} M_{k+1}
$$

será a perturbação. Nesse caso devemos ter $\lambda_{k} / 2 \gg \mu_{k, k+1}$.

$H_{0}$ tem dois estados estacionários correspondente ao autovalor $\Lambda_{0}^{(0)}=0$, ambos correspondem a dois sítios no mesmo estado (spins alinhados) com autovetor à esquerda

$$
{ }_{k}\left\langle\phi_{0}^{(0)}\right|=\left(\begin{array}{ll}
1 & 1
\end{array}\right)_{k}\left(\begin{array}{ll}
1 & 1
\end{array}\right)_{k+1} \quad \text { e } \quad{ }_{k}\left\langle\phi_{0^{\prime}}^{(0)}\right|=\left(\begin{array}{ll}
1 & 0
\end{array}\right)_{k}\left(\begin{array}{ll}
1 & 0
\end{array}\right)_{k+1} .
$$

O primeiro será dado por dois sítios recuperados

$$
\left|\psi_{0^{\prime}}^{(0)}\right\rangle_{k}=\left(\begin{array}{l}
1 \\
0
\end{array}\right)_{k}\left(\begin{array}{l}
1 \\
0
\end{array}\right)_{k+1},
$$

enquanto que o segundo por dois sítios infectados

$$
\left|\psi_{0}^{(0)}\right\rangle_{k}=\left(\begin{array}{l}
0 \\
1
\end{array}\right)_{k}\left(\begin{array}{l}
0 \\
1
\end{array}\right)_{k+1}
$$

(note que representamos o primeiro com o subíndice 0 e o segundo com $0^{\prime}$ ). Os dois autovetores à direita podem ser facilmente verificados associando-os às matrizes $R$ e $n . R$ diz respeito à taxa de infecção de um sítio recuperado, portanto se ambos os sítios estão infectados não pode haver infecção $\left(R_{k}\left|\psi_{0^{\prime}}^{(0)}\right\rangle_{k}=0\right)$. Por outro lado, $n$ verifica a existência de um sítio infectado, portanto aplicar $n$ num estado completamente 
recuperado nos retorna 0 .

Os estados com autovalor $\Lambda_{1}^{(0)}=\lambda_{k} / 2$ são

$$
\left|\psi_{1}^{(0)}\right\rangle_{k}=\left(\begin{array}{c}
1 \\
-1
\end{array}\right)_{k}\left(\begin{array}{l}
0 \\
1
\end{array}\right)_{k+1} \text { e }\left|\psi_{1^{\prime}}^{(0)}\right\rangle_{k}=\left(\begin{array}{l}
0 \\
1
\end{array}\right)_{k}\left(\begin{array}{c}
1 \\
-1
\end{array}\right)_{k+1}
$$

com autovetores à esquerda

$$
{ }_{k}\left\langle\phi_{1}^{(0)}\right|=\left(\begin{array}{ll}
1 & 0
\end{array}\right)_{k}\left(\begin{array}{ll}
0 & 1
\end{array}\right)_{k+1} \quad \text { e } \quad{ }_{k}\left\langle\phi_{1^{\prime}}^{(0)}\right|=\left(\begin{array}{ll}
0 & 1
\end{array}\right)_{k}\left(\begin{array}{ll}
1 & 0
\end{array}\right)_{k+1} .
$$

Para usar a teoria de perturbação, precisamos que os vetores sejam ortonormais, porém essa condição não é satisfeita para os vetores do estado estacionário dado que $\left\langle\phi_{0}^{(0)}\right|$ normaliza tanto $\left|\psi_{0}^{(0)}\right\rangle$ quanto $\left|\psi_{0^{\prime}}^{(0)}\right\rangle$. Para satisfazer essa condição, usaremos

$$
{ }_{k}\left\langle\phi_{0}^{(0)}\right|=\left(\begin{array}{ll}
1 & 1
\end{array}\right)_{k}\left(\begin{array}{ll}
1 & 1
\end{array}\right)_{k+1}-{ }_{k}\left\langle\phi_{0^{\prime}}^{(0)}\right|
$$

como autovetor à esquerda.

Pode ser verificado que a primeira correção é nula. Para o termo de segunda ordem basta apenas computar a correção em relação ao estado $\left|\psi_{0}^{(0)}\right\rangle$, pois a perturbação tem efeito nulo no outro. Calculando

$$
\left\langle\phi_{0}^{(0)}|V| \psi_{1}^{(0)}\right\rangle\left\langle\phi_{1}^{(0)}|V| \psi_{0}^{(0)}\right\rangle=-\mu_{k} \mu_{k+1}
$$

e

$$
\left\langle\phi_{0}^{(0)}|V| \psi_{1^{\prime}}^{(0)}\right\rangle\left\langle\phi_{1^{\prime}}^{(0)}|V| \psi_{0}^{(0)}\right\rangle=\mu_{k} \mu_{k+1},
$$

a correção em segunda ordem é dada por

$$
\Lambda_{0}^{(2)}=2 \frac{2 \mu_{k} \mu_{k+1}}{\lambda_{k}} .
$$

Em suma, a correção é nula caso os dois sítios estejam recuperados, ou $\Lambda_{0}^{(2)}$ se os dois sítios estiverem infectados. Da mesma forma que interpretamos a dizimação de um acoplamento no modelo de Ising, podemos introduzir um sítio com dois indivíduos com taxa de recuperação

$$
\tilde{\mu}=2 \frac{2 \mu_{k} \mu_{k+1}}{\lambda_{k}} .
$$


Portanto o gerador deste processo será

$$
H \approx \tilde{\mu} M+\sum_{\substack{i=1 \\ i \neq k}}^{N-1} \frac{\lambda_{i}}{2}\left[R_{i} n_{i+1}+R_{i+1} n_{i}\right]+\sum_{\substack{n=1 \\ i \neq k, k+1}}^{N} \mu_{i} M_{i},
$$

onde eliminamos um grau de liberdade.

\section{A.2.5 Equações de fluxo e comportamento crítico}

Antes de escrever as equações de fluxo, é conveniente reescrever as taxas $\tilde{\lambda}$ e $\tilde{\mu}$ em termos das variáveis $h=\kappa \mu$ e $J=\lambda / \kappa$ para obter as equações simétricas

$$
\tilde{J}=\tilde{\lambda}=\kappa \frac{J_{k} J_{k+1}}{h_{k}}
$$

$\mathrm{e}$

$$
\tilde{h}=\tilde{\mu}=\kappa \frac{h_{k} h_{k+1}}{J_{k}},
$$

com $\kappa=\sqrt{2}$. Comparando estas equações com (A.17) e (A.29), para o RTFI, podemos ver que elas diferem apenas pelo fator $\kappa$.

Seguindo o procedimento do SDRG, devemos escolher a maior taxa $\Omega=\max \{J, h\}$, dizimar este grau de liberdade e introduzir outro de acordo com (A.149) ou (A.150). Como consequência de dizimar a maior taxa, abaixávamos a taxa de energia em $d \Omega$. Todavia, como $\kappa>1$ no caso do CP, nem sempre abaixamos a escala de energia, isto é, se $\Omega$ não superar os outros valores de $J$ e $h$ por um fator $\kappa$, a escala de energia pode aumentar. Portanto nas equações a seguir vamos supor que a desordem é forte o suficiente para que (A.149) e (A.150) sempre abaixem a escala de energia.

As equações de fluxo são dadas por

$$
\frac{d P(h, \Omega)}{d \Omega}=P(h, \Omega)[R(\Omega, \Omega)-P(\Omega, \Omega)]-R(\Omega, \Omega) \int_{\frac{h}{\kappa}}^{\Omega} d h_{i} \frac{\Omega}{\kappa h_{i}} P\left(h_{i}, \Omega\right) P\left(\kappa \frac{\Omega h}{h_{i}}, \Omega\right)
$$

e

$$
\frac{d R(J, \Omega)}{d \Omega}=R(J, \Omega)[P(\Omega, \Omega)-R(\Omega, \Omega)]-P(\Omega, \Omega) \int_{\frac{J}{\kappa}}^{\Omega} d J_{i} \frac{\Omega}{\kappa J_{i}} R\left(J_{i}, \Omega\right) R\left(\kappa \frac{\Omega J}{J_{i}}, \Omega\right) .
$$

Usando os ansatz

$$
P(h, \Omega)=\frac{p_{0}(\Omega)}{\Omega}\left[\frac{\Omega}{h}\right]^{1-p_{0}} \quad \text { e } \quad R(J, \Omega)=\frac{r_{0}(\Omega)}{\Omega}\left[\frac{\Omega}{J}\right]^{1-r_{0}},
$$


obtemos para a $p_{0}$

$$
\frac{d}{d \Omega} \ln p_{0}-\ln \left(\frac{\Omega}{h}\right) \frac{d}{d \Omega} p_{0}=\frac{p_{0}}{\Omega}-\frac{r_{0} p_{0}}{\Omega} \kappa^{-p_{0}} \ln \left(\frac{\Omega \kappa}{h}\right),
$$

e para $r_{0}$

$$
\frac{d}{d \Omega} \ln r_{0}-\ln \left(\frac{\Omega}{J}\right) \frac{d}{d \Omega} r_{0}=\frac{r_{0}}{\Omega}-\frac{r_{0} p_{0}}{\Omega} \kappa^{-r_{0}} \ln \left(\frac{\Omega \kappa}{J}\right) .
$$

Devido ao fator $\kappa$, as equações acima são diferentes das apresentadas no modelo aleatório de Ising no campo transverso.

A medida que nos aproximamos do ponto fixo $(\Omega=0), p_{0}$ e $r_{0}$ tendem a zero, logo a solução assintótica pode ser obtida desprezando $r_{0} p_{0}$ (termo de segunda ordem) e a derivada em $p_{0}$ (a derivada em $\ln p_{0}$ varia mais rapidamente quando $p_{0} \rightarrow 0$ ), isso nos leva às equações

$$
\frac{d}{d \Gamma} \ln p_{0}=-p_{0} \quad \text { e } \quad \frac{d}{d \Gamma} \ln r_{0}=-r_{0},
$$

em termos de $\Gamma=-\ln \Omega$, cuja solução é

$$
p_{0}=r_{0}=\frac{1}{\Gamma-\Gamma_{0}}
$$

conforme no modelo de Ising. O mesmo argumento vale para $r_{0}$, pois as equações são simétricas.

Quando $p_{0}, r_{0} \rightarrow 0$, mas suficientemente longe do ponto fixo de forma que a derivada logarítmica possa ser desprezada e $\kappa^{-p_{0}}=\kappa^{-r_{0}} \approx 1$, obtemos

$$
[\Gamma-\ln h] \frac{d p_{0}}{d \Gamma}=p_{0}-r_{0} p_{0}[\ln (\Omega \kappa)-\ln h]
$$

$\mathrm{e}$

$$
[\Gamma-\ln J] \frac{d r_{0}}{d \Gamma}=r_{0}-r_{0} p_{0}[\ln (\Omega \kappa)-\ln J]
$$

Subtraindo uma equação da outra, podemos isolar os termos com $\ln J$ e $\ln h$ encontrando

$$
\left[\Gamma \frac{d}{d \Gamma}\left(p_{0}-r_{0}\right)+r_{0}-p_{0}\right]-\ln h\left[\frac{d p_{0}}{d \Gamma}+r_{0} p_{0}\right]-\ln J\left[\frac{d r_{0}}{d \Gamma}+r_{0} p_{0}\right]=0
$$


que só poderá ser satisfeita se

$$
\begin{aligned}
& \frac{d p_{0}}{d \Gamma}=-p_{0} r_{0}, \\
& \frac{d r_{0}}{d \Gamma}=-p_{0} r_{0},
\end{aligned}
$$

e

$$
\frac{d}{d \Gamma}\left(p_{0}-r_{0}\right)=\frac{p_{0}-r_{0}}{\Gamma} .
$$

Visto que as equações (A.156) e (A.157) são idênticas às obtidas no RTFI, a solução para o CP com desordem congelada é a mesma. Contudo, a última equação (A.158) afirma que esta solução é tão válida quanto mais próximos estamos do ponto fixo, isto é, quando $\Gamma \rightarrow \infty$, pois caso contrário (A.158) não será satisfeita.

Partindo da equação de fluxo (A.59), a equação para CP será

$$
\begin{aligned}
\frac{d}{d \Omega} \tilde{P}(h, \lambda, \Omega) & =\tilde{P}(h, \lambda, \Omega)[R(\Omega, \Omega)-P(\Omega, \Omega)]- \\
& -\tilde{R}(\Omega, \lambda, \Omega) \int_{\frac{h}{\kappa}}^{\Omega} d h_{i} \frac{\Omega}{\kappa h_{i}} \tilde{P}\left(h_{i}, \lambda, \Omega\right) \tilde{P}\left(\frac{\Omega h}{\kappa h_{i}}, \lambda, \Omega\right),
\end{aligned}
$$

e, usando o ansatz (A.61), chegamos em

$$
\pi \ln \left(\frac{\Omega}{h}\right)\left[\frac{d p_{l}}{d \Gamma}+\pi \rho \kappa^{-p_{l}}\right]-\left[\frac{d \pi}{d \Gamma}+\pi\left(p_{l}+p_{0}-r_{0}\right)\right]=\rho \pi^{2} \kappa^{-r_{l}} \ln \kappa
$$

que admite as mesmas soluções que o RTFI no regime assintótico $(\pi \rightarrow 0, \rho \rightarrow 0$ e $\Gamma \rightarrow$ $\infty)$. Devido à simetria, o mesmo argumento vale para a distribuição $\tilde{R}$. O procedimento é análogo para justificar a renormalização do número de infectados (momento magnético). 



\section{Apêndice B}

\section{Renormalização da equação logística com desordem temporal}

Neste apêndice realizaremos parte do cálculo do grupo de renormalização da equação logística com desordem temporal conforme a referência [87].,

\section{B.1 Equação logística com desordem temporal}

A equação logística é dada por

$$
\frac{d \rho(t)}{d t}=[\lambda-\mu] \rho-\lambda \rho^{2}
$$

cuja solução para valores constantes de $\lambda$ e $\mu$ é

$$
\rho(t)^{-1}=a(t) \rho_{0}^{-1}+c(t)
$$

$\operatorname{com} a=\exp ^{(\mu-\lambda) \Delta t}$ e $c=\lambda\left(e^{(\mu-\lambda) \Delta t}\right) /(\mu-\lambda)$.

Considere que $\mu$ e $\lambda$ mudem aleatoriamente no tempo, mas permaneçam constantes em intervalos de tempo $\left[t_{i+1}, t_{i}\right]$ de comprimento $\Delta t$. As variáveis aleatórias $\mu$ e $\lambda$ têm densidade de probabilidade $P(\lambda)$ and $R(\mu)$. Desta forma, a solução apresentada na seção anterior é válida em cada um dos intervalos de tempo $\Delta t$, e a densidade $\rho$ evolui de acordo com

$$
\rho_{i+1}^{-1}=a_{i} \rho_{i}^{-1}+c_{i}
$$

$\mathrm{Na}$ equação (B.3) o termo $a_{i}$ controla a variação exponencial da densidade em cada intervalo de tempo $\Delta t$, isto é, se $\lambda>\mu(\lambda<\mu)$ então $a_{i}$ é responsável pelo 
aumento (decaimento) exponencial de $\rho_{i}$. Portanto, a evolução densidade $\rho$ pode ser caracterizada pela variação exponencial da densidade em segmentos, ou intervalos de tempo, de tamanho $\Delta t . c_{i}$ só é importante quando a densidade $\rho_{i} \approx 1$ e impede que a densidade seja maior que 1 .

\section{B.2 Renormalização}

Primeiramente considere uma evolução de $\rho$ conforme (B.3), mas de forma que a densidade sempre aumente (diminua) após diminuir (aumentar). O processo de renormalização consiste em escolher o intervalo de tempo com menor variação na densidade, ou seja, o intervalo com $a_{i}$ mais próximo de 1 , e dizimá-lo. O processo de dizimação consiste em remover não só o intervalo $i$, mas também os seus vizinhos $i-1$ e $i+1$, e interpolar os pontos restantes usando (B.3). A interpolação será feita procurando por valores renormalizados $\bar{a}$ e $\bar{c}$ tais que

$$
\rho_{i+2}=\bar{a} \rho_{i-1}+\bar{c} .
$$

Utilizando (B.3) recursivamente é possível obter

$$
\begin{aligned}
\rho_{i+2} & =a_{i+1} \rho_{i+1}+c_{i+1} \\
& =a_{i+1}\left[a_{i} \rho_{i}+c_{i}\right]+c_{i+1} \\
& =a_{i+1}\left[a_{i}\left\{a_{i-1} \rho_{i-1}+c_{i-1}\right\}+c_{i}\right]+c_{i+1} \\
& =a_{i+1} a_{i} a_{i-1} \rho_{i-1}+a_{i+1}\left[a_{i} c_{i-1}+c_{i}\right]+c_{i+1} .
\end{aligned}
$$

Logo os valores renormalizados serão

$$
\bar{a}=a_{i+1} a_{i} a_{i-1} \quad \text { and } \quad \bar{c}=a_{i+1}\left[a_{i} c_{i-1}+c_{i}\right]+c_{i+1} .
$$

Após calcular a renormalização de $a_{i}$ e $c_{i}$, analisaremos a densidade de probabilidade de $a^{u p}$ and $a^{d n}$, que são definidas por

$$
a^{u p}=a_{i}, \quad \text { when } \quad \mu>\lambda
$$

e

$$
a^{d n}=a_{i}, \quad \text { if } \quad \mu<\lambda .
$$


O índice up e dn significa que a variável

$$
x=-\ln \rho
$$

aumenta ou diminui em cada iteração de (B.3).

No processo de dizimação também é conveniente definir

$$
\Omega=\min \left(a^{u p}-1,1 / a^{d n}-1\right) .
$$

Se $\Omega=a^{u p}$, a renormalização de $a_{i}$ será dada por

$$
\frac{1}{\bar{a}^{d n}}=\frac{\frac{1}{a_{i-1}^{d n}} \frac{1}{a_{i+1}^{d n}}}{\Omega} .
$$

Por outro lado, se $\Omega=1 / a^{d n}$,

$$
\bar{a}^{u p}=\frac{a_{i-1} a_{i+1}}{\Omega} .
$$

Devido à natureza multiplicativa de $\bar{a}$ é conveniente utilizar variáveis as logarítmicas

$$
\Gamma=\ln \Omega, \quad \zeta=-\ln a^{d n}-\Gamma \quad \text { and } \quad \beta=\ln a^{u p}-\Gamma .
$$

A partir destas definições, escrevemos as equações de fluxo das densidades de probabilidade $\mathcal{P}(\zeta, \Gamma)$ e $\mathcal{R}(\beta, \Gamma)$ de encontrar $\zeta$ ou $\beta$ dado o valor mínimo de variação de $\rho, \Omega$. A cada iteração do grupo de renormalização nós dizimamos o valor mínimo de $\Omega$ removendo $a_{i-1}, a_{i}$ e $a_{i+1}$, e consequentemente aumentamos $\Omega$ por $d \Omega(\Gamma, d \Gamma=d \Omega / \Omega)$. Logo as equações de fluxo são dadas por

$$
\begin{aligned}
D \mathcal{P}(\zeta-d \Gamma, \Gamma+d \Gamma) & =\mathcal{P}-\mathcal{P}(\zeta, \Gamma) \delta(\zeta) \\
& -2 d \Gamma \mathcal{R}_{0} \int_{0}^{\infty} d \zeta_{1} \mathcal{P}\left(\zeta_{1}, \Gamma\right) P(\zeta, \Gamma) \\
& +d \Gamma \mathcal{R}_{0} \int_{0}^{\infty} d \zeta_{1} \int_{0}^{\infty} d \zeta_{2} \mathcal{P}\left(\zeta_{1}, \Gamma\right) \mathcal{P}\left(\zeta_{2}, \Gamma\right) \delta\left(\zeta-\zeta_{1}-\zeta_{2}\right) \\
D \mathcal{P}(\zeta-d \Gamma, \Gamma+d \Gamma) & =\mathcal{P}-\mathcal{P}(\zeta, \Gamma) \delta(\zeta) \\
& -2 d \Gamma \mathcal{R}_{0} P(\zeta, \Gamma) \\
& +d \Gamma \mathcal{R}_{0} \int_{0}^{\zeta} d \zeta_{1} \mathcal{P}\left(\zeta_{1}, \Gamma\right) \mathcal{P}\left(\zeta-\zeta_{1}, \Gamma\right)
\end{aligned}
$$

A constante de renormalização $D$ pode ser obtida percebendo que, a cada iteração, o 
Apêndice B. Renormalização da equação logística com desordem temporal

número de segmentos up e dn diminui de $d \Gamma\left[\mathcal{R}_{0}+\mathcal{P}_{0}\right]$

$$
D=1-d \Gamma\left[\mathcal{R}_{0}+\mathcal{P}_{0}\right]
$$

Expandindo até a primeira ordem em $d \Gamma$ e longe de $\zeta=0$ obtemos

$$
\frac{\partial \mathcal{P}}{\partial \Gamma}-\frac{\partial \mathcal{P}}{\partial \zeta}=\mathcal{P}\left[\mathcal{R}_{0}+\mathcal{P}_{0}\right]-2 \mathcal{R}_{0} \mathcal{P}+\mathcal{R}_{0} \int_{0}^{\zeta} d \zeta_{1} \mathcal{P}\left(\zeta_{1}, \Gamma\right) \mathcal{P}\left(\zeta-\zeta_{1}, \Gamma\right)
$$

$\mathrm{Ou}$

$$
\frac{\partial \mathcal{P}}{\partial \Gamma}=\frac{\partial \mathcal{P}}{\partial \zeta}+\left[\mathcal{P}_{0}-\mathcal{R}_{0}\right] \mathcal{P}+\mathcal{R}_{0} \int_{0}^{\zeta} d \zeta_{1} \mathcal{P}\left(\zeta_{1}, \Gamma\right) \mathcal{P}\left(\zeta-\zeta_{1}, \Gamma\right)
$$

A equação para $\mathcal{R}$ pode ser obtida de forma análoga

$$
\frac{\partial \mathcal{R}}{\partial \Gamma}=\frac{\partial \mathcal{R}}{\partial \beta}+\left[\mathcal{R}_{0}-\mathcal{P}_{0}\right] \mathcal{R}+\mathcal{P}_{0} \int_{0}^{\beta} d \beta_{1} \mathcal{R}\left(\beta_{1}, \Gamma\right) \mathcal{R}\left(\beta-\beta_{1}, \Gamma\right) .
$$

A solução de interesse pode ser obtida através do ansatz

$$
\mathcal{R}=\mathcal{R}_{0} e^{-\mathcal{R}_{0} \beta}, \quad \mathcal{P}=\mathcal{P}_{0} e^{-\mathcal{P}_{0} \zeta} .
$$

Inserindo este ansatz na equação de fluxo de $\mathcal{P}$ encontramos

$$
\begin{gathered}
\frac{\partial \mathcal{P}_{0}}{\partial \Gamma}\left[e^{-\mathcal{P}_{0} \zeta}-\zeta \mathcal{P}\right]=-\mathcal{P}_{0} \mathcal{P}+\left[\mathcal{P}_{0}-\mathcal{R}_{0}\right] \mathcal{P}+\mathcal{R}_{0} \int_{0}^{\zeta} d \zeta_{1} \mathcal{P}_{0}^{2} e^{-\mathcal{P}_{0}} \\
\frac{\partial \mathcal{P}_{0}}{\partial \Gamma}\left[1-\zeta \mathcal{P}_{0}\right]=-\mathcal{P}_{0}^{2}+\left[\mathcal{P}_{0}-\mathcal{R}_{0}\right] \mathcal{P}_{0}+\mathcal{R}_{0} \mathcal{P}_{0}^{2} \zeta \\
\frac{\partial \mathcal{P}_{0}}{\partial \Gamma}\left[1-\zeta \mathcal{P}_{0}\right]=-\mathcal{R}_{0} \mathcal{P}_{0}\left[1-\mathcal{P}_{0} \zeta\right] \\
\frac{\partial \mathcal{P}_{0}}{\partial \Gamma}=-\mathcal{R}_{0} \mathcal{P}_{0} \quad \text { and } \quad \frac{\partial \mathcal{R}_{0}}{\partial \Gamma}=-\mathcal{R}_{0} \mathcal{P}_{0}
\end{gathered}
$$

No ponto crítico $\Gamma \rightarrow \infty$, esperamos que as duas densidades de probabilidade sejam simétricas $\mathcal{R}_{0}=\mathcal{P}_{0}, \log \mathrm{O}$

$$
\frac{\partial \mathcal{P}_{0}}{\partial \Gamma}=-\mathcal{P}_{0}^{2} \rightarrow \mathcal{P}_{0}=\frac{1}{\Gamma-\Gamma_{0}}=\frac{1}{\ln \Omega-\ln \Omega_{0}} .
$$


A densidade de probabilidade no ponto crítico e dada por

$$
\mathcal{P} \sim \frac{1}{\Gamma} e^{\frac{-\zeta}{\Gamma}}, \quad \Gamma \rightarrow \infty .
$$

Note que a densidade de probabilidade da distribuição de torna-se cada vez mais larga a cada interação do grupo de renormalização, justificando o termo ponto crítico de ruído infinito.

O número de intervalos de tempo diminui de acordo com

$$
\frac{d n}{d \Gamma}=-\left[\mathcal{P}_{0}+\mathcal{R}_{0}\right] n
$$

No ponto crítico, a solução desta equação é dada por

$$
n \sim \frac{1}{\left(\Gamma-\Gamma_{0}\right)^{2}} .
$$

Como o intervalo de tempo renormalizado é proporcional a $1 / n$, podemos encontrar

$$
<\bar{\Delta} t>\sim\left[\ln \frac{\Omega}{\Omega_{0}}\right]^{2} \text {. }
$$

Da equação diferencial para $\mathcal{P}_{0}$ e $\mathcal{R}_{0}$, podemos concluir que $\mathcal{P}_{0}-\mathcal{R}_{0}=2 \Delta$, onde $\Delta$ é uma constante. No ponto crítico, ambas as densidades de probabilidade $\mathcal{P}$ e $\mathcal{P}$ devem ser simétricas, portanto $\Delta$ tem a função de distância ao ponto crítico. Perto do ponto fixo temos $d \mathcal{P}_{0} / d \Gamma \approx 0, \operatorname{logo}$

$$
\mathcal{P}_{0} \sim \Delta \sim \frac{1}{\Gamma-\Gamma_{0}}
$$

que nos leva a

$$
<\bar{\Delta} t>\sim \Delta^{-\nu_{\|}}
$$

com o expoente crítico $\nu_{\|}=2$.

O número de partículas $N \sim \rho$ pode ser aproximado por $1 / a^{d n}$. Usando essa aproximação é possível obter

$$
\ln N \sim \int_{0}^{\infty} d \zeta \frac{\zeta+\Gamma}{\Gamma} e^{\frac{-\zeta}{\Gamma}}=2 \Gamma
$$

Logo, o tempo médio renormalizado será

$$
<\bar{\Delta} t>\sim[\ln N]^{2}
$$


Apesar da equação logística não conter noção de espaço, podemos definir um comprimento de correlação espacial através de $\xi \sim N^{1 / d}$

$$
<\bar{\Delta} t>\sim[\ln \xi]^{2}
$$

que é a versão temporal do escalamento ativado observado no processo de contato com desordem espacial congelada.

O comprimento da densidade de probabilidade de $x=-\ln \rho$ é proporcional à $-\ln a^{d n}$, e representa o quanto - $\ln \rho$ irá flutuar. since this later value represents how much $-\ln \rho$ Portanto

$$
\begin{aligned}
\Delta x & \sim \int_{0}^{\infty} \frac{\zeta+\Gamma}{\Gamma} e^{\frac{-\zeta}{\Gamma}} d \zeta \\
& \sim \int_{0}^{\infty} \frac{\zeta}{\Gamma} e^{\frac{-\zeta}{\Gamma}} d \zeta+\int_{0}^{\infty} e^{\frac{-\zeta}{\Gamma}} d \zeta \\
& \sim-\Gamma^{-1} \frac{\partial}{\partial \Gamma} \int_{0}^{\infty} e^{\frac{-\zeta}{\Gamma}} d \zeta+\int_{0}^{\infty} e^{\frac{-\zeta}{\Gamma}} d \zeta \\
& \sim-\Gamma^{-1} \frac{\partial}{\partial \Gamma^{-1}} \frac{1}{\Gamma^{-1}}+\Gamma=2 \Gamma
\end{aligned}
$$

A densidade de probabilidade de $x, P(x)$, pode ser obtida através do ansatz $P(x)=$ $\Phi(x / \Delta x) / \Delta x$. Desta densidade de probabilidade podemos calcular o valor médio de $\rho$, $\bar{\rho}=<\exp (-x)>$,

$$
\bar{\rho}=\int_{0}^{\infty} \frac{e^{-x}}{\Gamma} \Phi\left(\frac{-\ln \rho}{\Gamma}\right) d x .
$$

O comportamento assintótico é dado por

$$
\bar{\rho} \sim \frac{1}{\Gamma} \sim<\Delta t>^{-\delta} \sim \Delta^{\beta},
$$

com expoente crítico $\delta=1 / 2$ e $\beta=1$. 


\section{Referências Bibliográficas}

[1] Grassberger, P.: On the critical behavior of the general epidemic process and dynamical percolation. Mathematical Biosciences, 63(2):157 - 172, 1983, ISSN 0025-5564. http://www.sciencedirect.com/science/article/ pii/0025556482900360.

[2] Barabasi, Albert Laszlo e Stanley, Harry Eugene: Fractal Concepts in Surface Growth. Cambridge University Press, 1995.

[3] Ziff, Robert M., Gulari, Erdagon e Barshad, Yoav: Kinetic Phase Transitions in an Irreversible Surface-Reaction Model. Phys. Rev. Lett., 56:2553-2556, Jun 1986. http://link.aps.org/doi/10.1103/PhysRevLett.56.2553.

[4] Grassberger, Peter: Are damage spreading transitions generically in the universality class of directed percolation? Journal of Statistical Physics, 79(1):13-23, 1995, ISSN 1572-9613. http://dx.doi.org/10.1007/BF02179381.

[5] Takeuchi, Kazumasa A., Kuroda, Masafumi, Chaté, Hugues e Sano, Masaki: $D i$ rected Percolation Criticality in Turbulent Liquid Crystals. Phys. Rev. Lett., 99:234503, Dec 2007. http://link.aps.org/doi/10.1103/PhysRevLett.99. 234503.

[6] Tomé, Tânia e Oliveira, Mário José de: Dinâmica Estocástica e Irreversibilidade. Editora da Universidade de São Paulo (EDUSP), Brasil, São Paulo, SP, 2014.

[7] Ódor, Géza: Universality in Nonequilibrium Lattice Systems. World Scientific Publishing Co. Pte., London, Covent Garden, 2008.

[8] Henkel, Malte, Hinrinchsen, Haye e Lübeck, Sven: Non-Equilibrium Phase Transition. Springer Science + Business Media B. V., 3300 AA Dordrecht, The Netherlands, 2008. 
[9] Marro, Joaquín e Dickman, Ronald: Nonequilibrium Phase Transition in Lattice Models. Cambridge University Press, New York, 1999.

[10] Corte, Laurent, Chaikin, P. M., Gollub, J. P. e Pine, D. J. Nat Phys, 4, 2008. http: //www.nature.com/nphys/journal/v4/n5/suppinfo/nphys891_S1.html.

[11] Franceschini, Alexandre, Filippidi, Emmanouela, Guazzelli, Elisabeth e Pine, David J.: Transverse Alignment of Fibers in a Periodically Sheared Suspension: An Absorbing Phase Transition with a Slowly Varying Control Parameter. Phys. Rev. Lett., 107:250603, Dec 2011. https://link.aps.org/doi/10.1103/ PhysRevLett.107.250603.

[12] Okuma, S., Tsugawa, Y. e Motohashi, A.: Transition from reversible to irreversible flow: Absorbing and depinning transitions in a sheared-vortex system. Phys. Rev. B, 83:012503, Jan 2011. https://link.aps.org/doi/10.1103/PhysRevB.83. 012503.

[13] Korolev, K. S. e Nelson, David R.: Competition and Cooperation in OneDimensional Stepping-Stone Models. Phys. Rev. Lett., 107:088103, Aug 2011. https://link.aps.org/doi/10.1103/PhysRevLett.107.088103.

[14] Korolev, Kirill S., Xavier, João B., Nelson, David R. e Foster, Kevin R.: A Quantitative Test of Population Genetics Using Spatiogenetic Patterns in Bacterial Colonies. The American Naturalist, 178(4):538-552, 2011. http://dx.doi.org/ 10.1086/661897, PMID: 21956031.

[15] Ódor, Géza: Universality classes in nonequilibrium lattice systems. Rev. Mod. Phys., 76:663-724, Aug 2004. https://link.aps.org/doi/10.1103/ RevModPhys.76.663.

[16] Janssen, H. K.: On the nonequilibrium phase transition in reaction-diffusion systems with an absorbing stationary state. Zeitschrift für Physik B Condensed Matter, 42(2):151-154, 1981, ISSN 1431-584X. http://dx.doi.org/10.1007/ BF01319549.

[17] Grassberger, P.: On phase transitions in Schlögl's second model. Zeitschrift für Physik B Condensed Matter, 47(4):365-374, 1982, ISSN 1431-584X. http://dx. doi.org/10.1007/BF01313803.

[18] Stauffer, Dietrich e Aharony, Amnon: Introduction to Percolation Theory. Taylor \& Francis, London, Bristol, PA, 1994. 
[19] Souza, David R de, Tomé, Tânia e Ziff, Robert M: A new scale-invariant ratio and finite-size scaling for the stochastic susceptible-infected-recovered model. Journal of Statistical Mechanics: Theory and Experiment, 2011(03):P03006, 2011. http: //stacks.iop.org/1742-5468/2011/i=03/a=P03006.

[20] Tomé, Tânia e Ziff, Robert M.: Critical behavior of the susceptible-infectedrecovered model on a square lattice. Phys. Rev. E, 82:051921, Nov 2010. http: //link.aps.org/doi/10.1103/PhysRevE.82.051921.

[21] Hinrichsen, Haye: Non-equilibrium critical phenomena and phase transitions into absorbing states. Advances in Physics, 49(7):815-958, 2000. https://doi.org/ $10.1080 / 00018730050198152$.

[22] Jiménez-Dalmaroni, Andrea e Hinrichsen, Haye: Epidemic processes with immunization. Phys. Rev. E, 68:036103, Sep 2003. http://link.aps.org/doi/10. 1103/PhysRevE.68.036103.

[23] Grassberger, Peter, Chaté, Hugues e Rousseau, Guillaume: Spreading in media with long-time memory. Phys. Rev. E, 55:2488-2495, Mar 1997. http://Iink. aps.org/doi/10.1103/PhysRevE.55.2488.

[24] Tomé, Tânia e Oliveira, Mário J. de: Stationary distribution of finite-size systems with absorbing states. Phys. Rev. E, 72:026130, Aug 2005. http://Iink.aps. org/doi/10.1103/PhysRevE.72.026130.

[25] Wilson, Kenneth G.: Renormalization Group and Critical Phenomena. I. Renormalization Group and the Kadanoff Scaling Picture. Phys. Rev. B, 4:3174-3183, Nov 1971. http://link.aps.org/doi/10.1103/PhysRevB.4.3174.

[26] Harris, A B: Effect of random defects on the critical behaviour of Ising models. Journal of Physics C: Solid State Physics, 7(9):1671, 1974. http://stacks.iop. org $/ 0022-3719 / 7 / i=9 / a=009$.

[27] Vojta, Thomas e Dickman, Ronald: Spatiotemporal generalization of the Harris criterion and its application to diffusive disorder. Phys. Rev. E, 93:032143, Mar 2016. https://link.aps.org/doi/10.1103/PhysRevE.93.032143.

[28] Kinzel, W.: Phase transitions of cellular automata. Zeitschrift für Physik B Condensed Matter, 58(3):229-244, Sep 1985, ISSN 1431-584X. https://doi .org/ $10.1007 /$ BF01309255. 
[29] Alonso, J. J. e Muñoz, M. A.: Temporally disordered Ising models. EPL (Europhysics Letters), 56(4):485, 2001. http://stacks.iop.org/0295-5075/56/i=4/a= 485.

[30] Harris, T. E.: Contact Interactions on a Lattice. Ann. Probab., 2(6):969-988, dezembro 1974. http://dx.doi.org/10.1214/aop/1176996493.

[31] Dickman, Ronald: Reweighting in nonequilibrium simulations. Phys. Rev. E, 60:R2441-R2444, Sep 1999. https://link.aps.org/doi/10.1103/PhysRevE. 60. R2441.

[32] Dasgupta, Chandan e Ma, Shang keng: Low-temperature properties of the random Heisenberg antiferromagnetic chain. Phys. Rev. B, 22:1305-1319, Aug 1980. http: //link.aps.org/doi/10.1103/PhysRevB.22.1305.

[33] Ma, Shang keng, Dasgupta, Chandan e Hu, Chin kun: Random Antiferromagnetic Chain. Phys. Rev. Lett., 43:1434-1437, Nov 1979. http://link.aps.org/doi/ 10.1103/PhysRevLett.43.1434.

[34] Fisher, Daniel S.: Random transverse field Ising spin chains. Phys. Rev. Lett., 69:534-537, Jul 1992. http://link.aps.org/doi/10.1103/PhysRevLett.69. 534.

[35] Fisher, Daniel S.: Critical behavior of random transverse-field Ising spin chains. Phys. Rev. B, 51:6411-6461, Mar 1995. http://link.aps.org/doi/10.1103/ PhysRevB.51.6411.

[36] Hooyberghs, Jef, Iglói, Ferenc e Vanderzande, Carlo: Strong Disorder Fixed Point in Absorbing-State Phase Transitions. Phys. Rev. Lett., 90:100601, Mar 2003. http://link.aps.org/doi/10.1103/PhysRevLett.90.100601.

[37] Hooyberghs, Jef, Iglói, Ferenc e Vanderzande, Carlo: Absorbing state phase transitions with quenched disorder. Phys. Rev. E, 69:066140, Jun 2004. http: //Iink.aps.org/doi/10.1103/PhysRevE.69.066140.

[38] Hoyos, José A.: Weakly disordered absorbing-state phase transitions. Phys. Rev. E, 78:032101, Sep 2008. https://link.aps.org/doi/10.1103/PhysRevE.78. 032101.

[39] Ibrahim, Ahmed K., Barghathi, Hatem e Vojta, Thomas: Enhanced rare-region effects in the contact process with long-range correlated disorder. Phys. Rev. 
E, 90:042132, Oct 2014. https://link.aps.org/doi/10.1103/PhysRevE.90. 042132.

[40] P., Verhulst. Corresp. Math. Phys., (10):113-121, 1838.

[41] Tomé, Tânia e Oliveira, Mário J de: Susceptible-infected-recovered and susceptibleexposed-infected models. Journal of Physics A: Mathematical and Theoretical, 44(9):095005, 2011. http://stacks.iop.org/1751-8121/44/i=9/a=095005.

[42] Alexandrowicz, Z.: Critically branched chains and percolation clusters. Physics Letters A, 80(4):284 - 286, 1980, ISSN 0375-9601. http://www. sciencedirect. com/science/article/pii/0375960180900237.

[43] Jacobsen, Jesper Lykke: Critical points of Potts and $O(N)$ models from eigenvalue identities in periodic Temperley-Lieb algebras. Journal of Physics A: Mathematical and Theoretical, 48(45):454003, 2015. http://stacks.iop.org/1751-8121/48/ $i=45 / a=454003$.

[44] Wada, Alexander H O, Tomé, Tânia e Oliveira, Mário J de: Critical properties of the susceptible-exposed-infected model on a square lattice. Journal of Statistical Mechanics: Theory and Experiment, 2015(4):P04014, 2015. http://stacks.iop. org/1742-5468/2015/i=4/a=P04014.

[45] Wada, Alexander H. O.: Simulações Numéricas da Percolação Dinâmica. Tese de Mestrado, Instituto de Física da Universidade de São Paulo, 2015.

[46] Wada, Alexander H O e Oliveira, Mário J de: Critical properties of the contact process with quenched dilution. Journal of Statistical Mechanics: Theory and Experiment, 2017(4):043209, apr 2017. https://doi.org/10.1088\%2F1742-5468\% 2Faa694b.

[47] Vojta, Thomas, Farquhar, Adam e Mast, Jason: Infinite-randomness critical point in the two-dimensional disordered contact process. Phys. Rev. E, 79:011111, Jan 2009. http://link.aps.org/doi/10.1103/PhysRevE.79.01111.

[48] Oliveira, Marcelo M de e Ferreira, Silvio C: Universality of the contact process with random dilution. Journal of Statistical Mechanics: Theory and Experiment, 2008(11):P11001, 2008. http://stacks.iop.org/1742-5468/2008/i=11/ $\mathrm{a}=\mathrm{P} 11001$. 
[49] Dahmen, Silvio R, Sittler, Lionel e Hinrichsen, Haye: Multicritical behaviour of the diluted contact process. Journal of Statistical Mechanics: Theory and Experiment, 2007(01):P01011, 2007. http://stacks.iop.org/1742-5468/2007/i= $01 / \mathrm{a}=\mathrm{P} 01011$

[50] Miyazaki, Ryoji e Nishimori, Hidetoshi: Real-space renormalization-group approach to the random transverse-field Ising model in finite dimensions. Phys. Rev. E, 87:032154, Mar 2013. http://link.aps.org/doi/10.1103/PhysRevE. 87.032154.

[51] Motrunich, Olexei, Mau, Siun Chuon, Huse, David A. e Fisher, Daniel S.: Infiniterandomness quantum Ising critical fixed points. Phys. Rev. B, 61:1160-1172, Jan 2000. http://link.aps.org/doi/10.1103/PhysRevB.61.1160.

[52] Xu, Xiao, Wang, Junfeng, Lv, Jian Ping e Deng, Youjin: Simultaneous analysis of three-dimensional percolation models. Frontiers of Physics, 9(1):113-119, Feb 2014, ISSN 2095-0470. https://doi.org/10.1007/s11467-013-0403-z.

[53] Ballesteros, H G, Fernández, L A, Martín-Mayor, V, Sudupe, A Muñoz, Parisi, G e Ruiz-Lorenzo, J J: Scaling corrections: site percolation and Ising model in three dimensions. Journal of Physics A: Mathematical and General, 32(1):1, 1999. http://stacks.iop.org/0305-4470/32/i=1/a=004.

[54] Deng, Youjin e Blöte, Henk W. J.: Monte Carlo study of the site-percolation model in two and three dimensions. Phys. Rev. E, 72:016126, Jul 2005. https: //link.aps.org/doi/10.1103/PhysRevE.72.016126.

[55] Xu, Xiao, Wang, Junfeng, Lv, Jian Ping e Deng, Youjin: Simultaneous analysis of three-dimensional percolation models. Frontiers of Physics, 9(1):113-119, 2014, ISSN 2095-0470. http://dx.doi.org/10.1007/s11467-013-0403-z.

[56] Dammer, Stephan M e Hinrichsen, Haye: Spreading with immunization in high dimensions. Journal of Statistical Mechanics: Theory and Experiment, 2004(07):P07011, 2004. http://stacks.iop.org/1742-5468/2004/i=07/a= P07011.

[57] Grassberger, P: Numerical studies of critical percolation in three dimensions. Journal of Physics A: Mathematical and General, 25(22):5867, 1992. http:// stacks. iop.org/0305-4470/25/i=22/a=015. 
[58] Brown, Robert: XXVII. A brief account of microscopical observations made in the months of June, July and August 1827, on the particles contained in the pollen of plants; and on the general existence of active molecules in organic and inorganic bodies. The Philosophical Magazine, 4(21):161-173, 1828. https: //doi.org/10.1080/14786442808674769.

[59] Metzler, Ralf e Klafter, Joseph: The random walk's guide to anomalous diffusion: a fractional dynamics approach. Physics Reports, 339(1):1 - 77, 2000, ISSN 0370-1573. http://www.sciencedirect.com/science/article/ pii/S0370157300000703.

[60] Höfling, Felix e Franosch, Thomas: Anomalous transport in the crowded world of biological cells. Reports on Progress in Physics, 76(4):046602, 2013. http: //stacks. iop.org/0034-4885/76/i=4/a=046602.

[61] Bressloff, Paul C. e Newby, Jay M.: Stochastic models of intracellular transport. Rev. Mod. Phys., 85:135-196, Jan 2013. https://link.aps.org/doi/10.1103/ RevModPhys . 85.135.

[62] Metzler, Ralf, Jeon, Jae Hyung, Cherstvy, Andrey G. e Barkai, Eli: Anomalous diffusion models and their properties: non-stationarity, non-ergodicity, and ageing at the centenary of single particle tracking. Phys. Chem. Chem. Phys., 16:2412824164, 2014. http://dx.doi.org/10.1039/C4CP03465A.

[63] Meroz, Yasmine e Sokolov, Igor M.: A toolbox for determining subdiffusive mechanisms. Physics Reports, 573:1 - 29, 2015, ISSN 0370-1573. http://www. sciencedirect.com/science/article/pii/s0370157315001404, A toolbox for determining subdiffusive mechanisms.

[64] Metzler, R., Jeon, J. H. e Cherstvy, A.G.: Non-Brownian diffusion in lipid membranes: Experiments and simulations. Biochimica et Biophysica Acta (BBA) - Biomembranes, 1858(10):2451 - 2467, 2016, ISSN 0005-2736. http: //www.sciencedirect.com/science/article/pii/S0005273616300219, Biosimulations of lipid membranes coupled to experiments.

[65] Norregaard, Kamilla, Metzler, Ralf, Ritter, Christine M., Berg-Sørensen, Kirstine e Oddershede, Lene B.: Manipulation and Motion of Organelles and Single Molecules in Living Cells. Chemical Reviews, 117(5):4342-4375, 2017. https: //doi.org/10.1021/acs. chemrev.6b00638, PMID: 28156096. 
[66] Einstein, A.: Investigations on the Theory of the Brownian Movement. Dover, New York, 1956.

[67] Xie, X. Sunney, Choi, Paul J., Li, Gene Wei, Lee, Nam Ki e Lia, Giuseppe: SingleMolecule Approach to Molecular Biology in Living Bacterial Cells. Annual Review of Biophysics, 37(1):417-444, 2008. https://doi.org/10.1146/annurev . biophys.37.092607.174640, PMID: 18573089.

[68] Bräuchle, C., Lamb, D. C. e Michaelis, J.: Single Particle Tracking and Single Molecule Energy Transfer. Wiley - VCH, Weinheim, 2012.

[69] Manzo, Carlo e Garcia-Parajo, Maria F: A review of progress in single particle tracking: from methods to biophysical insights. Reports on Progress in Physics, 78(12):124601, 2015. http://stacks.iop.org/0034-4885/78/i=12/a=124601.

[70] Kahane, J. P.: Some Random Series of Functions. Cambridge University Press, London, 1985.

[71] Yaglom, A. M.: Correlation Theory of Stationary and Related Random Functions. Springer, Heidelberg, 1987.

[72] Beran, J.: Statistics for Long-Memory Processes. Chapman \& Hall, New York, 1994.

[73] Biagini, F., Y. Hu, B. Øksendal e Zhang, T.: Stochastic Calculus for Fractional Brownian Motion and Applications. Springer, Berlin, 2008.

[74] Chakravarti, Nalini e Sebastian, K.L.: Fractional Brownian motion models for polymers. Chemical Physics Letters, 267(1):9 - 13, 1997, ISSN 0009-2614. http: //www.sciencedirect.com/science/article/pii/s0009261497000754.

[75] Panja, Debabrata: Generalized Langevin equation formulation for anomalous polymer dynamics. Journal of Statistical Mechanics: Theory and Experiment, 2010(02):L02001, 2010. http://stacks.iop.org/1742-5468/2010/i=02/ $\mathrm{a}=\mathrm{L} 02001$.

[76] Simonsen, Ingve: Measuring anti-correlations in the nordic electricity spot market by wavelets. Physica A: Statistical Mechanics and its Applications, 322:597 - 606, 2003, ISSN 0378-4371. http://www.sciencedirect.com/science/article/ pii/S0378437102019386. 
[77] Szymanski, Jedrzej e Weiss, Matthias: Elucidating the Origin of Anomalous Diffusion in Crowded Fluids. Phys. Rev. Lett., 103:038102, Jul 2009. https: //link.aps.org/doi/10.1103/PhysRevLett.103.038102.

[78] Mikosch, Thomas, Resnick, Sidney, Rootzén, Holger e Stegeman, Alwin: Is Network Traffic Appriximated by Stable Lévy Motion or Fractional Brownian Motion? Ann. Appl. Probab., 12(1):23-68, fevereiro 2002. https://doi.org/10.1214/aoap/ 1015961155.

[79] Hansen, Alex, Engøy, Thor e Måløy, Knut Jørgen: MEASURING HURST EXPONENTS WITH THE FIRST RETURN METHOD. Fractals, 02(04):527-533, 1994. https://doi.org/10.1142/S0218348X94000740.

[80] Ding, Mingzhou e Yang, Weiming: Distribution of the first return time in fractional Brownian motion and its application to the study of on-off intermittency. Phys. Rev. E, 52:207-213, Jul 1995. https://link.aps.org/doi/10.1103/PhysRevE. 52.207.

[81] Krug, J., Kallabis, H., Majumdar, S. N., Cornell, S. J., Bray, A. J. e Sire, C.: Persistence exponents for fluctuating interfaces. Phys. Rev. E, 56:2702-2712, Sep 1997. https://link.aps.org/doi/10.1103/PhysRevE.56.2702.

[82] Molchan, G. M.: Maximum of a Fractional Brownian Motion: Probabilities of Small Values. Communications in Mathematical Physics, 205(1):97-111, Aug 1999, ISSN 1432-0916. https://doi.org/10.1007/s002200050669.

[83] Wada, Alexander H. O. e Vojta, Thomas: Fractional Brownian motion with a reflecting wall. Phys. Rev. E, 97:020102, Feb 2018. https://link.aps.org/doi/ 10.1103/PhysRevE.97.020102.

[84] Wada, Alexander H O, Warhover, Alex e Vojta, Thomas: Non-Gaussian behavior of reflected fractional Brownian motion. Journal of Statistical Mechanics: Theory and Experiment, 2019(3):033209, mar 2019. https://doi.org/10.1088\% 2F $1742-5468 \% 2 \mathrm{Fab} 02 \mathrm{f} 1$.

[85] Qian, Hong: Fractional Brownian Motion and Fractional Gaussian Noise, páginas 22-33. Springer Berlin Heidelberg, Berlin, Heidelberg, 2003, ISBN 978-3-540-44832-7. https://doi.org/10.1007/3-540-44832-2_2. 
[86] Makse, Hernán A., Havlin, Shlomo, Schwartz, Moshe e Stanley, H. Eugene: Method for generating long-range correlations for large systems. Phys. Rev. E, 53:54455449, May 1996. https://link.aps.org/doi/10.1103/PhysRevE.53.5445.

[87] Vojta, Thomas e Hoyos, José A.: Infinite-noise criticality: Nonequilibrium phase transitions in fluctuating environments. EPL (Europhysics Letters), 112(3):30002, 2015. http://stacks.iop.org/0295-5075/112/i=3/a=30002.

[88] Hinrichsen, Haye: Non-equilibrium critical phenomena and phase transitions into absorbing states. Advances in Physics, 49(7):815-958, 2000. https://doi.org/ $10.1080 / 00018730050198152$.

[89] Vazquez, Federico, Bonachela, Juan A., López, Cristóbal e Muñoz, Miguel A.: Temporal Griffiths Phases. Phys. Rev. Lett., 106:235702, Jun 2011. https: //link.aps.org/doi/10.1103/PhysRevLett.106.235702.

[90] Wada, Alexander H. O., Small, Matthew e Vojta, Thomas: Extinction transitions in correlated external noise. Phys. Rev. E, 98:022112, Aug 2018. https://link. aps.org/doi/10.1103/PhysRevE.98.022112.

[91] Vojta, Thomas e Dickman, Ronald: Spatiotemporal generalization of the Harris criterion and its application to diffusive disorder. Phys. Rev. E, 93:032143, Mar 2016. https://Iink.aps.org/doi/10.1103/PhysRevE.93.032143.

[92] Noest, André J.: New universality for spatially disordered cellular automata and directed percolation. Phys. Rev. Lett., 57:90-93, Jul 1986. http://link.aps. org/doi/10.1103/PhysRevLett.57.90.

[93] Vojta, T.: Rare region effects at classical, quantum, and non-equilibrium phase transitions. J. Phys. A, 39:R143, 2006.

[94] Iglói, Ferenc: Exact renormalization of the random transverse-field Ising spin chain in the strongly ordered and strongly disordered Griffiths phases. Phys. Rev. B, 65:064416, Jan 2002. http://link.aps.org/doi/10.1103/PhysRevB. 65.064416 .

[95] Grassberger, P e Torre, A de la: Reggeon field theory (Schlögl's first model) on a lattice: Monte Carlo calculations of critical behaviour. Annals of Physics, 122(2):373 - 396, 1979, ISSN 0003-4916. http://www. sciencedirect.com/ science/article/pii/0003491679902070. 
[96] Cardy, J L e Grassberger, P: Epidemic models and percolation. Journal of Physics A: Mathematical and General, 18(6):L267, 1985. http://stacks.iop.org/ $0305-4470 / 18 / i=6 / a=001$.

[97] Vojta, Thomas: Rare region effects at classical, quantum and nonequilibrium phase transitions. Journal of Physics A: Mathematical and General, 39(22):R143, 2006. http://stacks.iop.org/0305-4470/39/i=22/a=R01.

[98] Aharony, Amnon e Harris, A. Brooks: Absence of Self-Averaging and Universal Fluctuations in Random Systems near Critical Points. Phys. Rev. Lett., 77:37003703, Oct 1996. http://link.aps.org/doi/10.1103/PhysRevLett.77.3700.

[99] Vojta, Thomas e Dickison, Mark: Critical behavior and Griffiths effects in the disordered contact process. Phys. Rev. E, 72:036126, Sep 2005. http://Iink. aps . org/doi/10.1103/PhysRevE.72.036126.

[100] Karevski, D., Lin, Y C., Rieger, H., Kawashima, N. e Iglói, F.: Random quantum magnets with broad disorder distribution. The European Physical Journal B Condensed Matter and Complex Systems, 20(2):267-276, 2001, ISSN 1434-6036. http://dx.doi.org/10.1007/PL00011100.

[101] Vojta, Thomas e Lee, Man Young: Nonequilibrium Phase Transition on a Randomly Diluted Lattice. Phys. Rev. Lett., 96:035701, Jan 2006. http://Iink.aps . org/doi/10.1103/PhysRevLett.96.035701.

[102] L Rodrigues Áttila, Chatelain, Christophe, Tomé, Tânia e Oliveira, Mário J de: Critical behavior in lattice models with two symmetric absorbing states. Journal of Statistical Mechanics: Theory and Experiment, 2015(1):P01035, 2015. http: //stacks.iop.org/1742-5468/2015/i=1/a=P01035.

[103] Leães Rodrigues Áttila: Estudo de transições de fase em sistemas com simetria "up-down"e estados absorventes. Tese de Doutoramento, Instituto de Física da Universidade de São Paulo, 2014.

[104] Fisher, Daniel S.: Random antiferromagnetic quantum spin chains. Phys. Rev. B, 50:3799-3821, Aug 1994. http://link.aps.org/doi/10.1103/PhysRevB. 50. 3799.

[105] Moreira, Adriana G. e Dickman, Ronald: Critical dynamics of the contact process with quenched disorder. Phys. Rev. E, 54:R3090-R3093, Oct 1996. http://Iink. aps.org/doi/10.1103/PhysRevE.54.R3090. 
[106] Dickman, Ronald e Moreira, Adriana G.: Violation of scaling in the contact process with quenched disorder. Phys. Rev. E, 57:1263-1268, Feb 1998. http://link. aps.org/doi/10.1103/PhysRevE.57.1263.

[107] Grassberger, P: Numerical studies of critical percolation in three dimensions. Journal of Physics A: Mathematical and General, 25(22):5867, 1992. http:// stacks.iop.org/0305-4470/25/i=22/a=015.

[108] Wang, Junfeng, Zhou, Zongzheng, Zhang, Wei, Garoni, Timothy M. e Deng, Youjin: Bond and site percolation in three dimensions. Phys. Rev. E, 87:052107, May 2013. http://link.aps.org/doi/10.1103/PhysRevE.87.052107. 